\title{
Metal-Micelle Cooperativity: Phosphine Ligand-Free Ultrasmall Palladium(II) Nanoparticles for Oxidative Mizoroki-Heck-Type Couplings in Water at Room Temperature
}

Tharique N. Ansari, ${ }^{\dagger}$ Jacek B. Jasinski, ${ }^{\Perp}$ David K. Leahy, ${ }^{*}$ and Sachin Handa ${ }^{\dagger *}$

'Department of Chemistry, University of Louisville, Louisville, Kentucky 40292, United States

"Materials Characterization, Conn Center for Renewable Energy Research, University of Louisville, Louisville, KY, 40292, USA

*Process Chemistry Development, Takeda Pharmaceuticals International, Cambridge, Massachusetts 02139, USA

\section{*sachin.handa@,louisville.edu \\ *david.k.leahy@me.com}

\section{Supplementary Material}

\section{Contents}

Page

1. General experimental details

S1

2. Synthesis and isolation of nanoparticles

S2

3. Detailed analysis of nanoparticles

S3-S11

4. Effect of stirring rate on reaction outcome

S12-S14

5. Filtration \& extraction studies

S15-S16

6. Three-phase test

S17

7. Reaction optimizations

S18-S20

8. Optimized procedure for catalytic couplings

S21

9. Substrate scope

S22

10. Multi-gram scale reaction

$\mathrm{S} 23$

11. Analytical Data

S24-S33

12. Reference

S34

13. NMR Spectra

S35-S71 


\section{GENERAL EXPERIMENTAL DETAILS}

All manipulations were carried out under air unless otherwise noted. Solvent molarity listed in reaction schemes is relative to the limiting reagent.

\subsection{Chemicals and Supply}

Chromatography. TLC plates (UV 254 indicator, aluminum backed, 175-225 $\mu \mathrm{m}$ thickness, standard grade silica gel, 230-400 mesh) was supplied by Merck; silica gel (60 Å pore size, 230400 mesh) was purchased from Silicycle; sand was purchased from Fisher Chemical.

Solvents. Ethyl acetate, hexanes, toluene and HPLC-grade water were purchased from Fisher Chemical. NMR solvents were obtained from Sigma-Aldrich; surfactant solutions were prepared in HPLC-grade water; PS-750-M was prepared as previously reported; ${ }^{1}$ poly(ethylene glycol) methyl ether 750 (mPEG-750-M) was supplied by Alfa-Aesar.

Reagents. $\mathrm{Pd}(\mathrm{OAc})_{2}$ was supplied by Sigma-Aldrich; Arylboronic acids were purchased from Sigma-Aldrich or Combi-Blocks or Matrix Scientific; Commercially available styrenes were either supplied by Sigma-Aldrich or Combi-Blocks Inc. or Oakwood Chemicals. $p$-benzoquinone was purchased from Fisher Chemicals. Acryloyl Wang resin and methyl acrylate was purchased from Sigma-Aldrich.

General Instrumentation. All products were purified by flash chromatography using a Teledyne Isco CombiFlash Rf 150 or manual column using silica gel (60 Å pore size, 230-400 mesh). GCMS data was obtained using a Thermo Scientific Trace 1300 Gas Chromatograph coupled with a Thermo Scientific ISQ-QD Single Quadrupole Mass Spectrometer. NMR spectra were recorded at $23{ }^{\circ} \mathrm{C}$ on Varian MR-400 and Varian Unity INOVA 500 (400 and $500 \mathrm{MHz}$, respectively). Reported chemical shifts are referenced to residual solvent peaks. ${ }^{2}$ Infrared absorbance spectra were acquired on a FTIR Perkin Elmer Spectrum Two: UATR Two spectrometer using $1 \mathrm{~cm}^{-1}$ resolution. HRMS analyses were obtained either using a 5975C Mass Selective Detector coupled with a 7890A Gas Chromatograph (Agilent Technologies) or orbit-trap. Melting points were determined using a Thomas Hoover melting point apparatus with samples in Kimble Kimex 51 capillaries $(1.5-1.8 \times 90 \mathrm{~mm})$. 


\section{SYNTHESIS AND ISOLATION OF NANOPARTICLES}

In a $50 \mathrm{~mL}$ round-bottom flask containing a PTFE-coated stir bar, nanoparticle precursor palladium(II)acetate $(85 \mathrm{mg}, 0.38 \mathrm{mmol}$ ) was added. Later, $25 \mathrm{~mL} 3 \mathrm{wt} \%$ aq. PS-750-M was added, and the round-bottom flask was closed with a rubber septum. The reaction mixture was transferred to a pre-heated oil bath at $45^{\circ} \mathrm{C}$ and stirred for the next 50 minutes. The coloration of the suspension was changed from light brown to the darker brown. After 50 minutes, the reaction mixture was removed from the oil both and allowed to cool to rt. The obtained dark brown suspension was used as the nanoparticle stock solution (SS-1). These nanoparticles were characterized using IR spectroscopy, HRTEM, EDAX, SEM, and HRMS. The nanoparticles can be stored without any notable precautions. 


\section{DETAILED ANALYSIS OF NANOPARTICLES}

\subsection{Analysis by IR spectroscopy}

(a) IR Spectroscopy. The nanoparticles suspended in $3 \mathrm{wt} \%$ aq. PS-750-M were prepared according to the procedure provided in section 2 (page S2). $\sim 100 \mu \mathrm{L}$ aliquot was withdrawn from the nanoparticle solution and analyzed using FTIR Perkin Elmer spectrophotometer.

Table S1. Sample details for IR experiments

\begin{tabular}{c|c}
\hline Sample ID & Constituents \\
\hline Sample-A & $\operatorname{Pd}(\mathrm{OAc})_{2}$ \\
Sample-B & $\sim 50-100 \mu \mathrm{L}$ of 3 wt $\%$ aq. PS-750-M \\
Sample-C & $\sim 100 \mu \mathrm{L}$ of nanoparticles \\
\hline
\end{tabular}

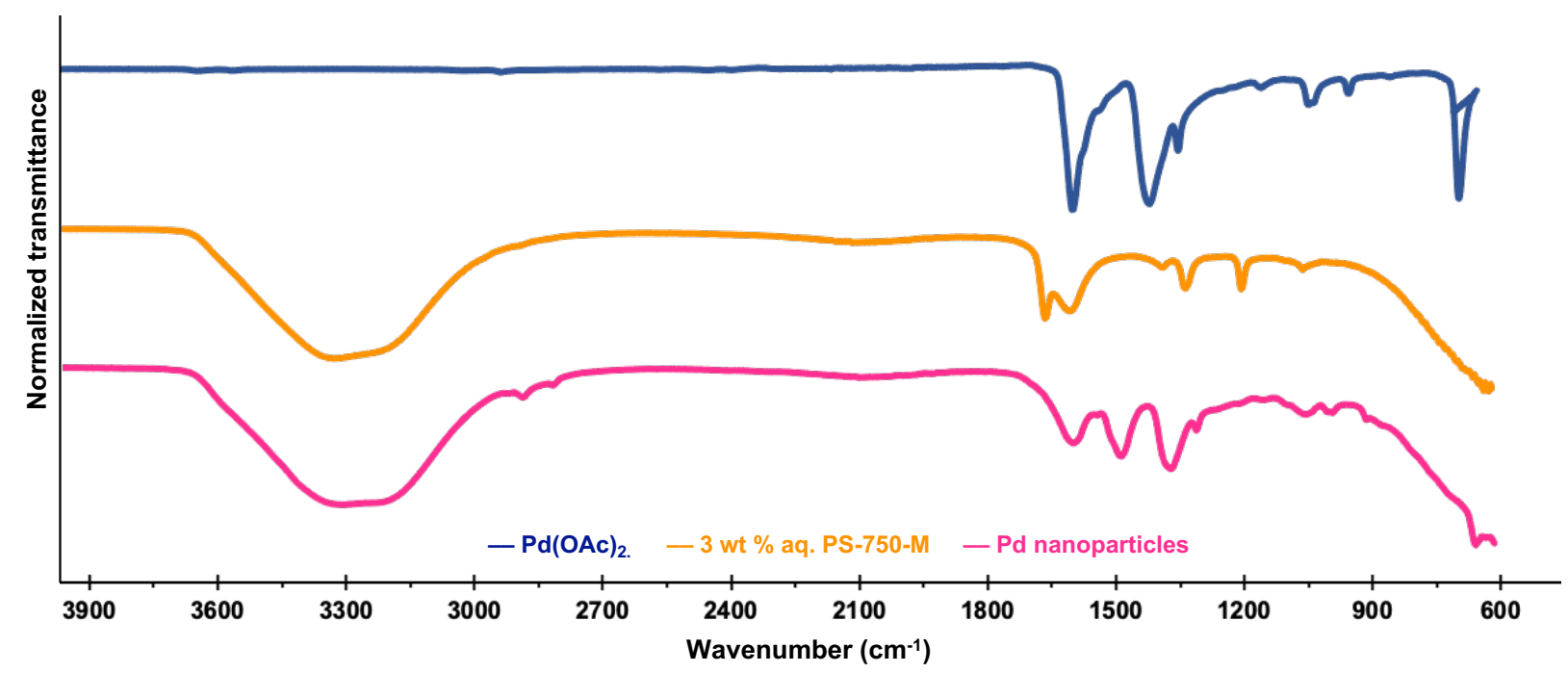

Figure S1. IR spectra of NPs (bottom) compared to $\mathrm{Pd}(\mathrm{OAc})_{2}$ and PS-750-M.

3.2 HRTEM \& SEM analysis of the nanoparticles. The nanoparticles were prepared according to the procedure provided in section 2 (page S2). The sample was prepared by diluting $0.1 \mathrm{~mL}$ of nanoparticle stock solution with $0.4 \mathrm{~mL}$ of DI $\mathrm{H}_{2} \mathrm{O}$. 

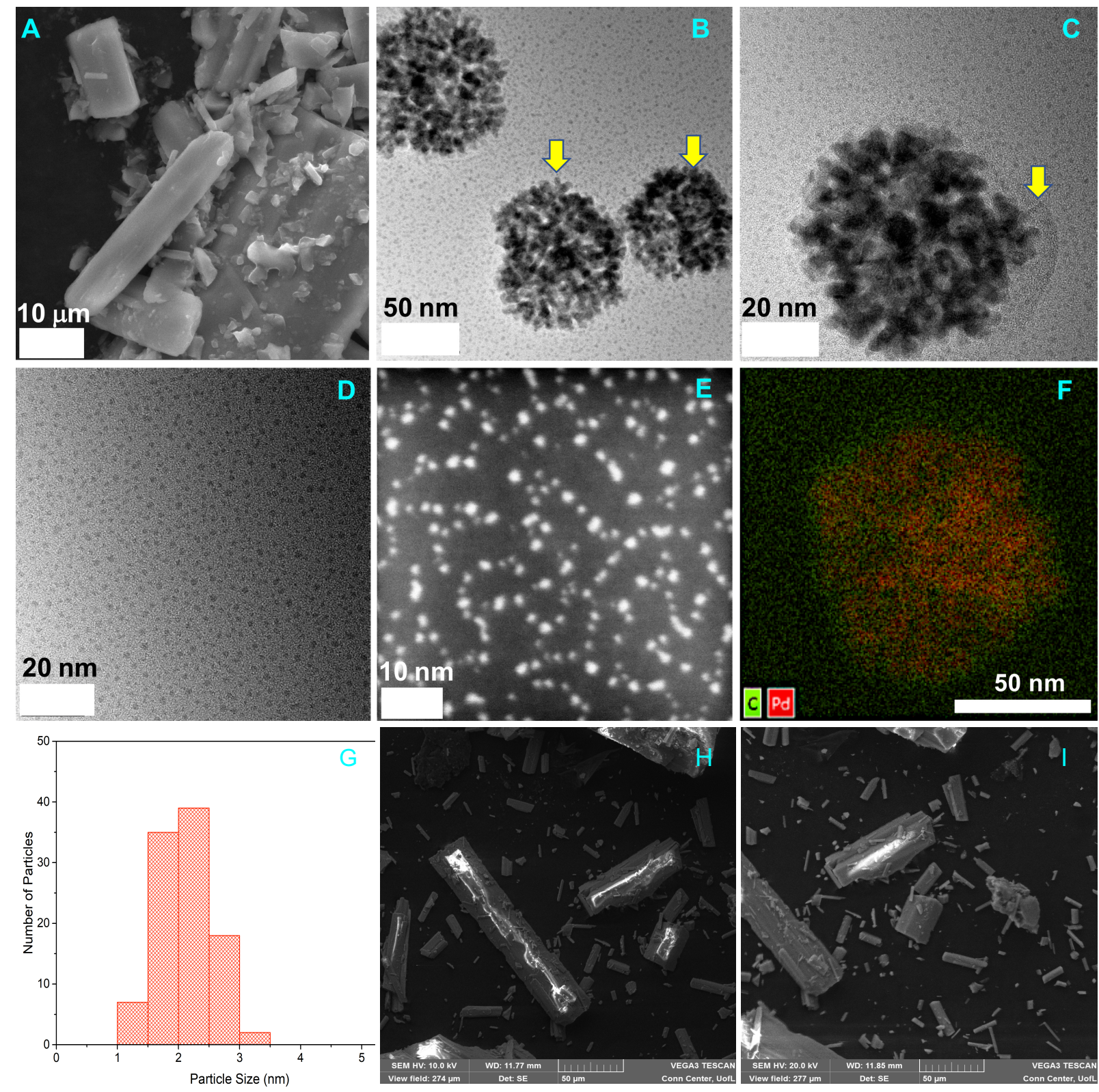

Figure S2. (A) SEM analysis of $\mathrm{Pd}(\mathrm{OAc})_{2}$ coated with PS-750-M, (B-D) HRTEM analysis, (E) STEM-HAADF of Pd(II) NPs, (F) EDAX analysis of Pd(II) NPs, (G) Histogram of UltrasmallPd nanoparticles, (H, \& I) SEM analysis of $\mathrm{Pd}(\mathrm{OAc})_{2}$. 


\subsection{Substrate interaction with nanoparticles-NMR investigation}

(a) Sample preparation. All samples were stirred at room temperature for 45 minutes.

Table S2. Sample details for NMR experiments

\begin{tabular}{c|c}
\hline Sample ID & Constituents \\
\hline Sample-A & 2-fluoroboronic acid (1) \\
Sample-B & 1 equiv. 2-fluoroboronic acid + 1 equiv. nanoparticles \\
Sample-C & 4-flurostyrene (2) \\
Sample-D & 1 equiv. 4-flurostyrene +1 equiv. nanoparticles \\
Sample-E & 1 equiv. 4-flurostyrene + 1 equiv. nanoparticles + 1 equiv. 2-fluoroboronic acid \\
\hline
\end{tabular}

(b) NMR experiments. All experiments were carried out in Varian Innova $400 \mathrm{MHz}$ NMR at 25 ${ }^{\circ} \mathrm{C}, \mathrm{D}_{2} \mathrm{O}$ as NMR solvent and 4-trifluoromethylbromobenzene as internal standard. ${ }^{3}$

(Note: The internal standard was prepared in a capillary tube by dissolving 4trifluoromethylbromobenzene in $\mathrm{CDCl}_{3}$, and tip of capillary tube was permanently sealed) 

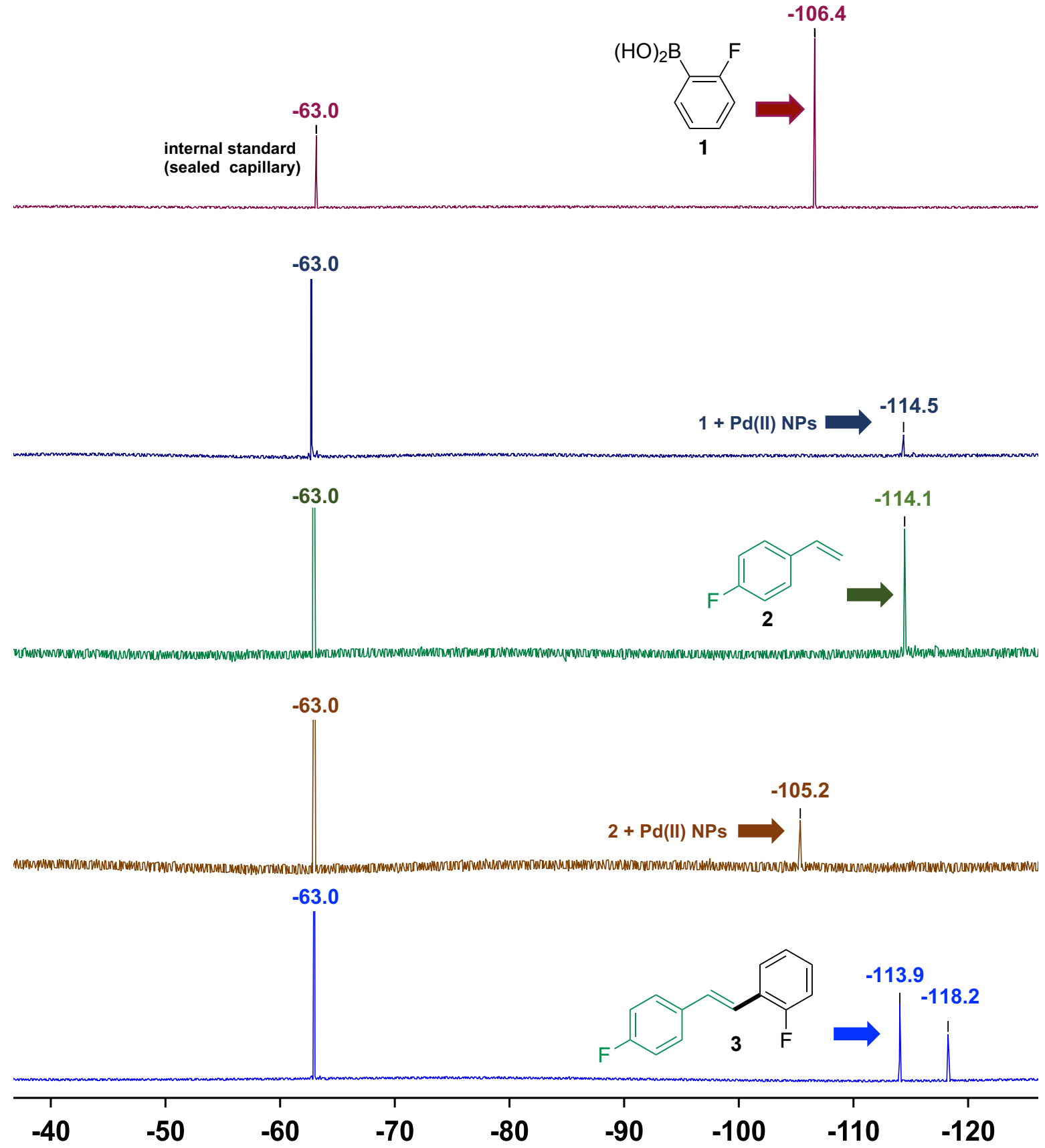

Figure S3. NMR analysis of nanoparticle interaction with the substrates. 


\subsection{XPS analysis of the catalyst}
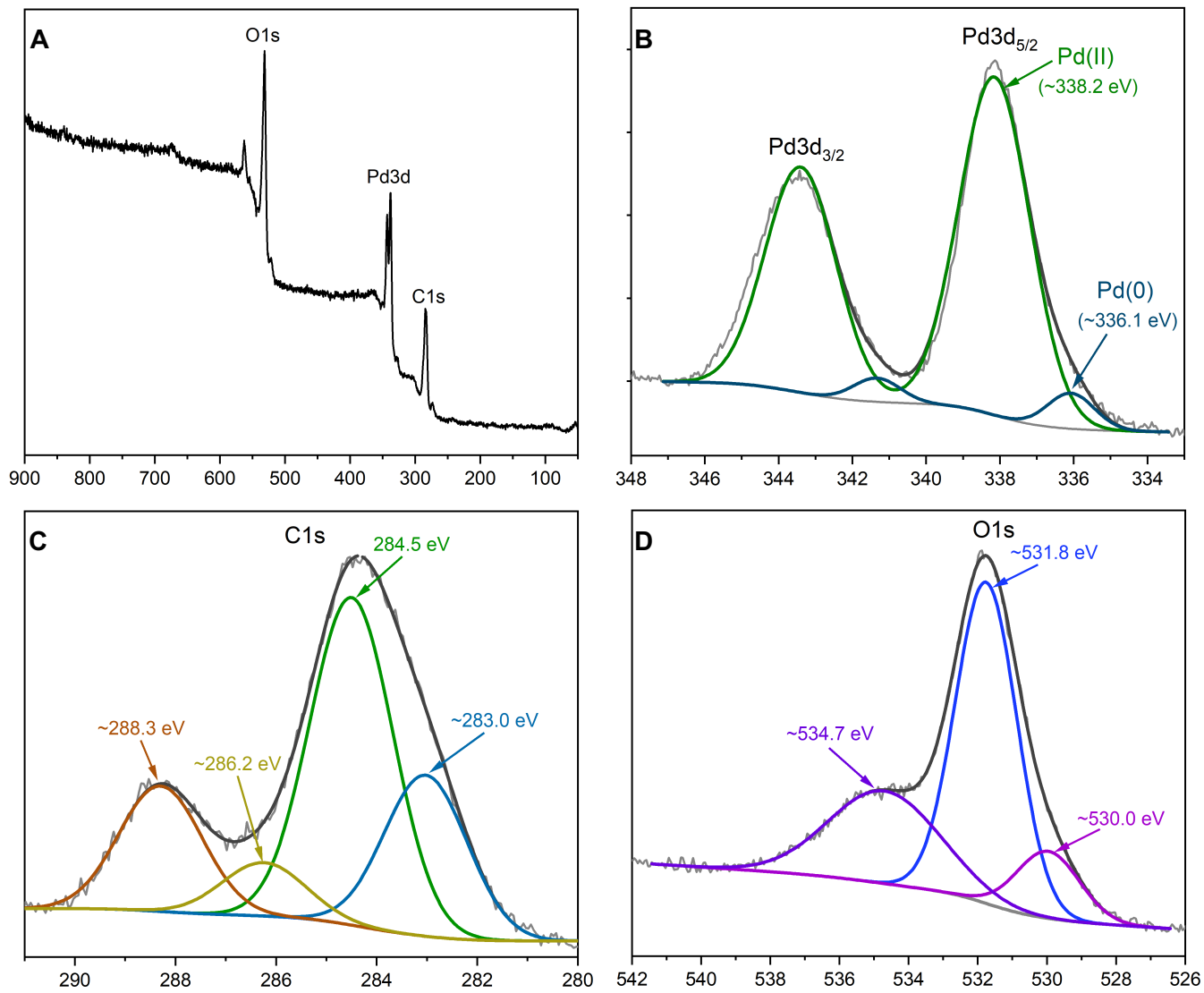

Figure S4. XPS analysis of the nanocatalyst. (A) Survey spectrum of the nanocatalyst, (B) Peak deconvolution of high-resolution Pd3d spectrum, (C) Peak deconvolution of high-resolution $\mathrm{C} 1 \mathrm{~s}$ spectrum, (D) Peak deconvolution of high-resolution O1s spectrum. Note: The trace Pd(0) is present is likely due to reduction of Pd(II) under heat generated from X-ray and presence of acetate and hydroxide ions.

The nanoparticles were prepared according to the procedure described in section 2, and the solid nanoparticles were obtained after removing water under reduced pressure and drying the sample overnight under vacuum. X-ray photoelectron spectroscopy (XPS) studies were performed using a VG Scientific MultiLab 3000 ultra-high vacuum surface analysis system, equipped with a with dual-anode (Mg/Al) X-ray source and a CLAM4 hemispherical electron energy analyzer. The measurements were conducted at the base pressure in the $10^{-9}$ Torr range and a nonmonochromatized $\mathrm{Al} \mathrm{K}_{\alpha} \mathrm{X}$-ray radiation $(\mathrm{h} v \approx 1486.6 \mathrm{eV})$ was used as the X-ray source. XPS spectra were collected at an electron emission angle of $54.7^{\circ}$ relative to the surface normal. For the binding energy (BE) calibration, the high-resolution spectrum of $\mathrm{C} 1 \mathrm{~s}$ was collected, deconvoluted, and the $\mathrm{C}-\mathrm{C}$ peak of adventitious carbon set to $284.5 \mathrm{eV}$. Fig.S4.A represents the 
survey spectrum of the nanocatalyst and Fig.S4.B shows the peak de-convolution of the highresolution spectrum of $\mathrm{Pd} 3 \mathrm{~d}$ with main component (93\% of total intensity, green curve), and its $\operatorname{Pd} 3 \mathrm{~d}_{5 / 2}$ at $\sim 338.2 \mathrm{eV}$ corresponds to the $\mathrm{Pd}(\mathrm{II})$. There is also a residual component $(7 \%$ of total intensity, blue curve) with its $\operatorname{Pd} 3 \mathrm{~d}_{5 / 2}$ at $\sim 336.1 \mathrm{eV}$, that most likely corresponds to $\operatorname{Pd}(0)$ formed due to slight reduction (possibly due to X-ray beam effects). Fig.S4.C and Fig.S4.D represent the peak deconvolution of the high-resolution spectrum of $\mathrm{C} 1 \mathrm{~s}$ and $\mathrm{O} 1 \mathrm{~s}$ respectively.

\subsection{HRMS analysis of the catalyst}

The nanoparticle precursor $\mathrm{Pd}(\mathrm{OAc})_{2}$ was analyzed using high resolution mass spectrometry (ESI/APCI Orbitrap). This analysis indicated the presence of trimeric palladium species $\mathrm{Pd}_{3}(\mathrm{OAc})_{5}^{+}$.

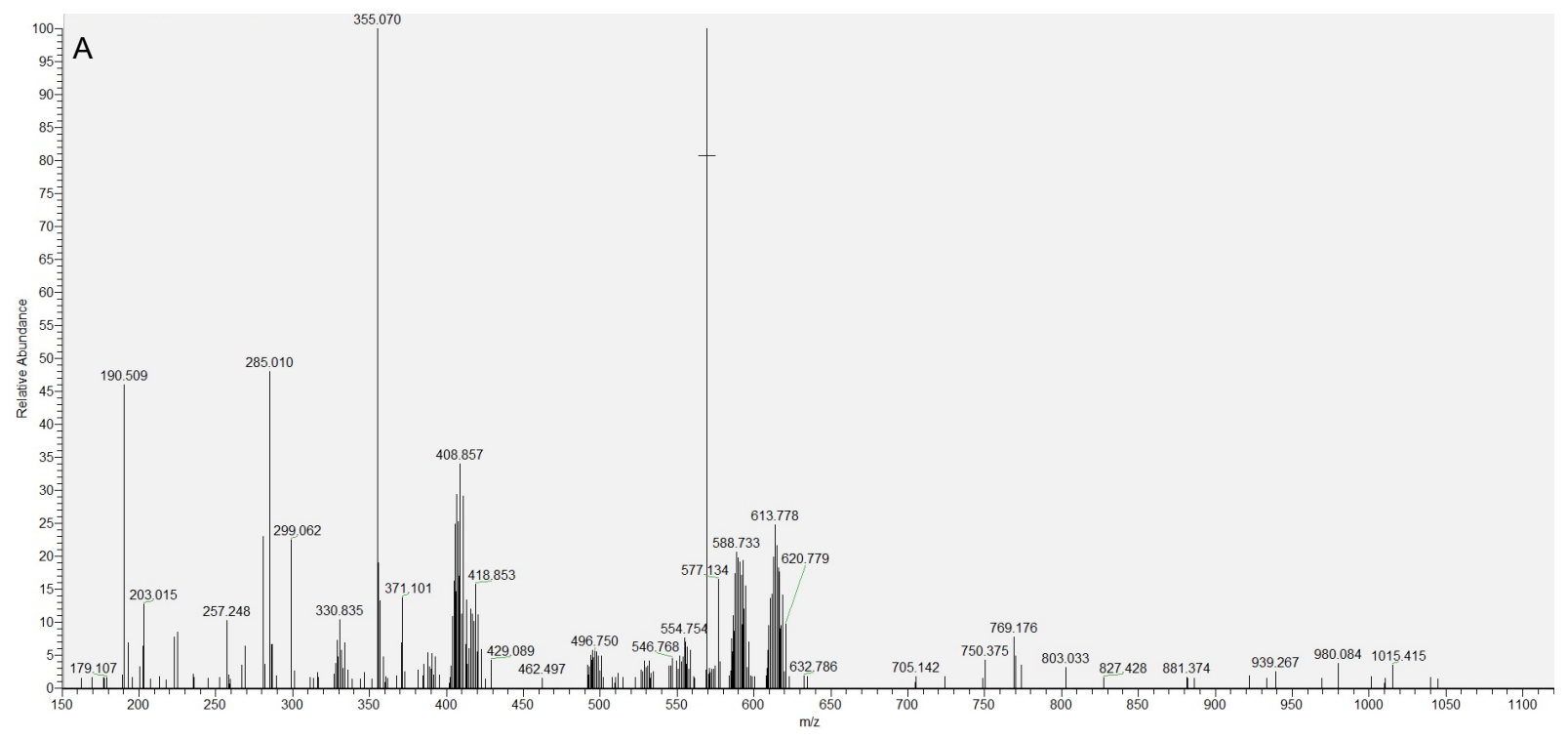




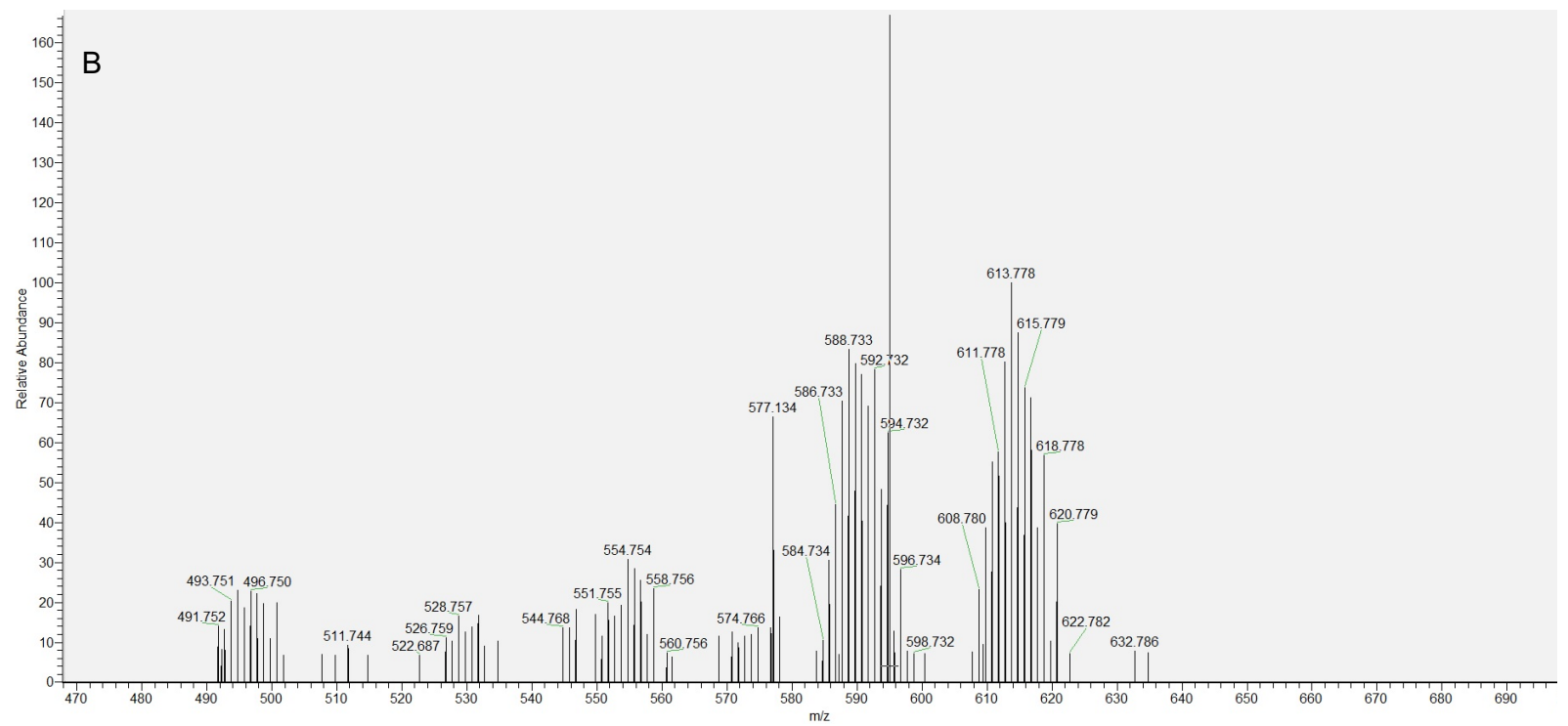

Figure S5. HRMS analysis of the nanocatalyst. (A) HRMS full spectrum of $\mathrm{Pd}(\mathrm{OAc})_{2},(\mathrm{~B})$ $\mathrm{Pd}_{3}(\mathrm{OAc}){ }_{5}{ }^{+} \mathrm{HRMS}$.

The nanoparticles were prepared according to procedure described in section 2 (page S2), the obtained nanoparticles solution was diluted with $0.3 \mathrm{~mL}$ of $\mathrm{CH}_{3} \mathrm{CN}$, and the resulting solution was analyzed at $150{ }^{\circ} \mathrm{C}$ infusion temperature. The exact structure of the NPs wasn't able to confirm with this analysis, but it is clear that the $\mathrm{Pd}^{+2}$ ions are associated with the amphiphile based on the fragmentation pattern.

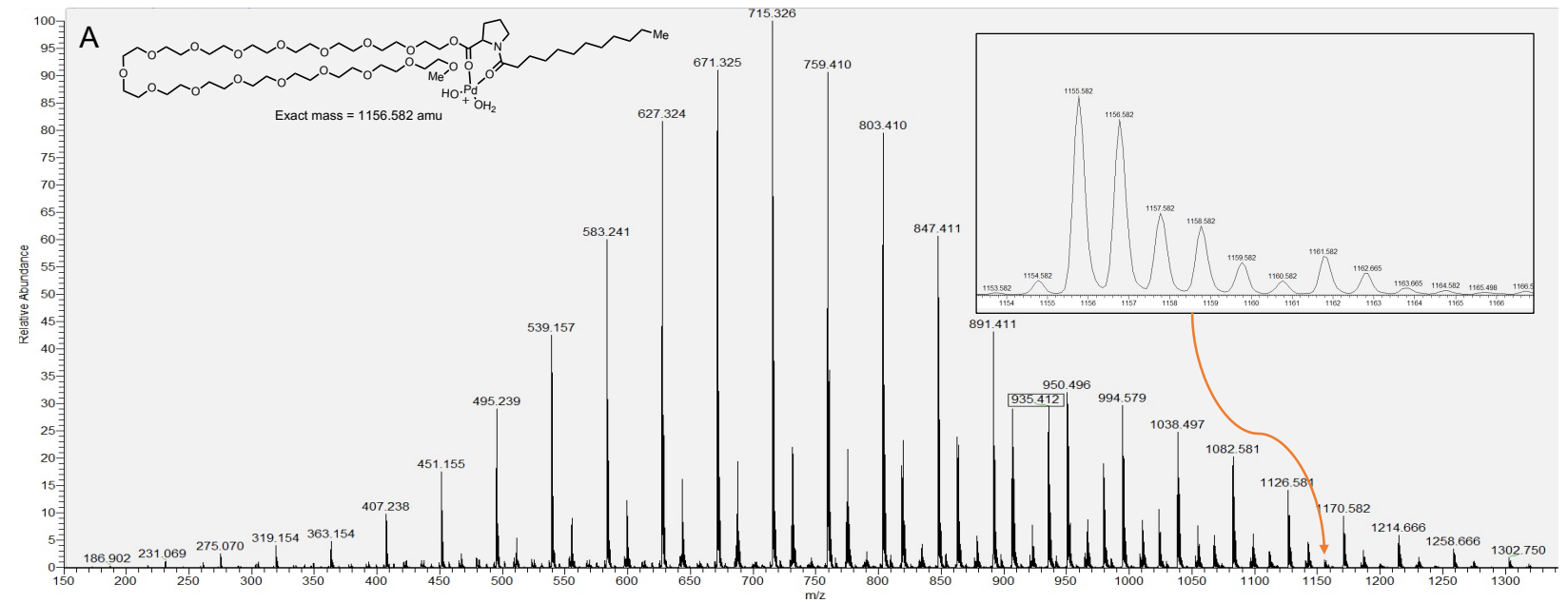



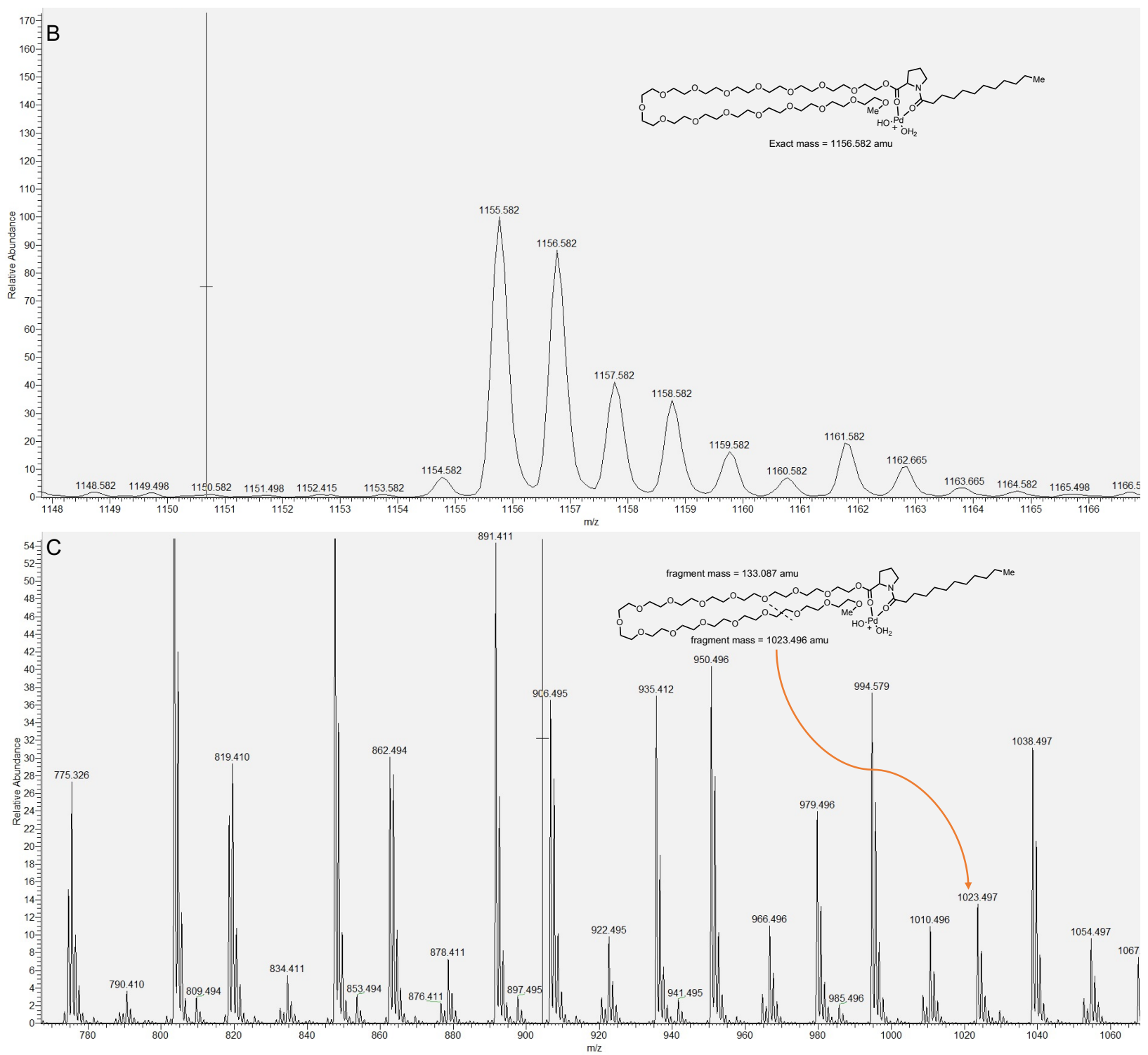


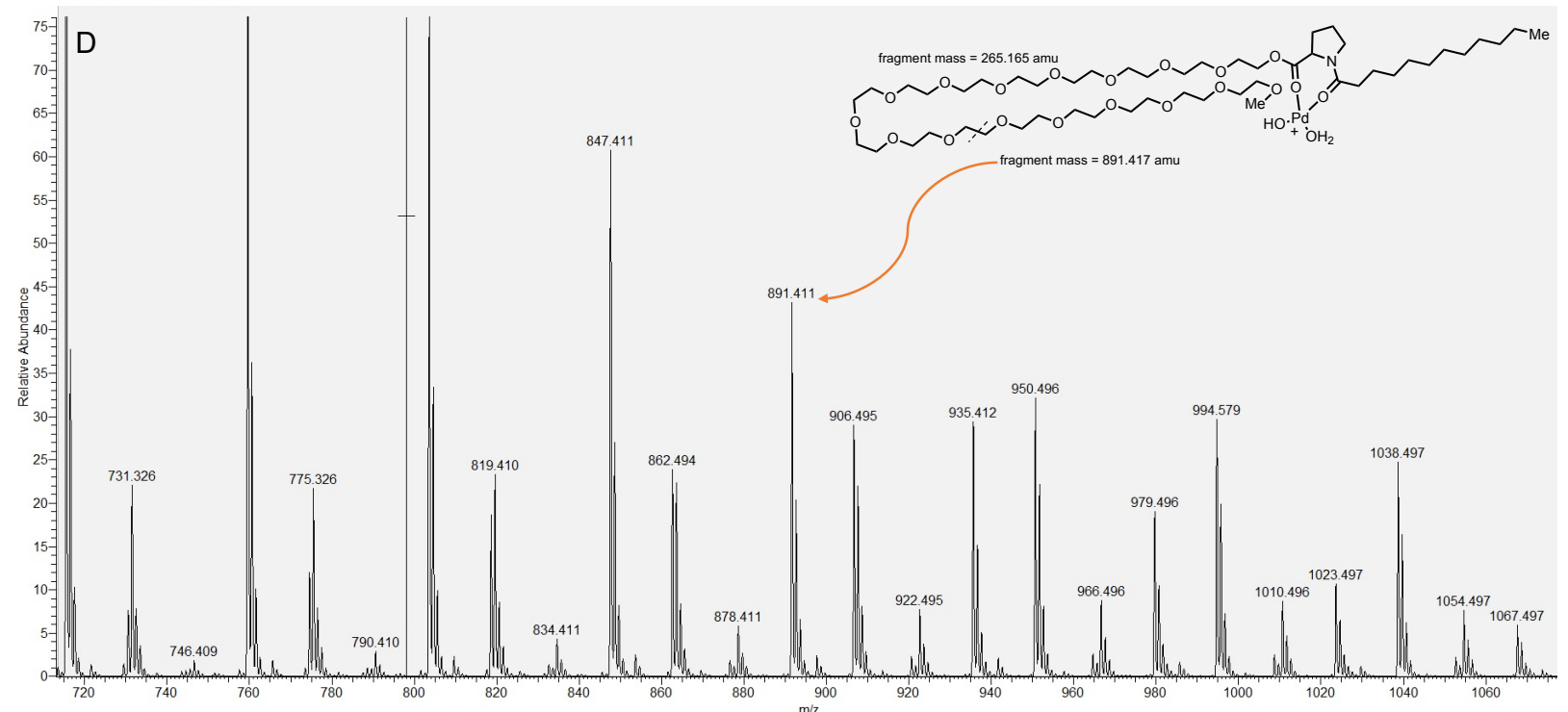

Figure S6. HRMS analysis of the nanocatalyst, (A) HRMS full spectrum of the NPs, (B) expansion of HRMS analysis NPs, (C, D) fragments from HRMS analysis. 


\section{EFFECT OF STIRRING RATE ON REACTION OUTCOME}

To study the effect of stirring rates on ultra-small nanoparticle generation, and its impact on catalytic activity, the coupling between 4-trifluoromethyl phenylboronic acid (4) and styrene (5) was performed under standard conditions. Five different stirring rates were chosen (a) $50 \mathrm{rpm}$, (b) $100 \mathrm{rpm}$, (c) 500, (d) 1000, and (e) $1500 \mathrm{rpm}$.

\subsection{Procedure-Stirring rate $0,50 \mathrm{rpm}$}

A $4.0 \mathrm{~mL}$ reaction vial containing a PTFE-coated magnetic stir bar was charged with nanoparticle precursor palladium(II)acetate $(1.7 \mathrm{mg}, 0.0075 \mathrm{mmol})$. Later, $1 \mathrm{~mL} 3 \mathrm{wt} \%$ aq. PS-750-M was added, and the vial was closed screwcap. Then, the reaction vial was transferred to a pre-heated stir plate at $45^{\circ} \mathrm{C}$ and stirred for 50 minutes. After cooling to room temperature, the reaction vial was opened, and arylboronic acid $(0.275 \mathrm{mmol}), p$-benzoquinone $(108 \mathrm{mg}, 0.375 \mathrm{mmol})$, and styrene $(0.25 \mathrm{mmol})$ were added. The reaction vial was closed with a screwcap and then stirred $(50 \mathrm{rpm})$ at room temperature. Such six reactions were kept in parallel. Every 2 hours one of the six reactions were analyzed using GCMS after the addition of mesitylene $(35.0 \mu \mathrm{L}, 0.25 \mathrm{mmol}$, internal standard) and $1.0 \mathrm{~mL}$ of EtOAc.

\subsection{Procedure-Stirring rate $@ 100 \mathrm{rpm}$}

A $4.0 \mathrm{~mL}$ reaction vial containing a PTFE-coated magnetic stir bar was charged with nanoparticle precursor palladium(II)acetate $(1.7 \mathrm{mg}, 0.0075 \mathrm{mmol})$. Later, $1 \mathrm{~mL} 3 \mathrm{wt} \%$ aq. PS-750-M was added, and the vial was closed screwcap. Then, the reaction vial was transferred to a pre-heated stir plate at $45^{\circ} \mathrm{C}$ and stirred for 50 minutes. After cooling to room temperature, the reaction vial was opened, and arylboronic acid $(0.275 \mathrm{mmol}), p$-benzoquinone $(108 \mathrm{mg}, 0.375 \mathrm{mmol})$, and styrene $(0.25 \mathrm{mmol})$ were added. The reaction vial was closed with a screwcap and then stirred $(100 \mathrm{rpm})$ at room temperature. Such six reactions were kept in parallel. Every 2 hours one of the six reactions were analyzed using GCMS after the addition of mesitylene $(35.0 \mu \mathrm{L}, 0.25 \mathrm{mmol}$, internal standard) and $1.0 \mathrm{~mL}$ of EtOAc.

\subsection{Procedure-Stirring rate $@ 500 \mathrm{rpm}$}

A $4.0 \mathrm{~mL}$ reaction vial containing a PTFE-coated magnetic stir bar was charged with nanoparticle precursor palladium(II)acetate $(1.7 \mathrm{mg}, 0.0075 \mathrm{mmol})$. Later, $1 \mathrm{~mL} 3 \mathrm{wt} \%$ aq. PS-750-M was added, and the vial was closed screwcap. Then, the reaction vial was transferred to a pre-heated stir plate at $45^{\circ} \mathrm{C}$ and stirred for 50 minutes. After cooling to room temperature, the reaction vial was opened, and arylboronic acid $(0.275 \mathrm{mmol}), p$-benzoquinone $(108 \mathrm{mg}, 0.375 \mathrm{mmol})$, and 
styrene $(0.25 \mathrm{mmol})$ were added. The reaction vial was closed with a screwcap and then stirred $(500 \mathrm{rpm})$ at room temperature. Such six reactions were kept in parallel. Every 2 hours one of the six reactions were analyzed using GCMS after the addition of mesitylene $(35.0 \mu \mathrm{L}, 0.25 \mathrm{mmol}$, internal standard) and $1.0 \mathrm{~mL}$ of EtOAc.

\subsection{Procedure-Stirring rate @ 1000 rpm}

A $4.0 \mathrm{~mL}$ reaction vial containing a PTFE-coated magnetic stir bar was charged with nanoparticle precursor palladium(II)acetate $(1.7 \mathrm{mg}, 0.0075 \mathrm{mmol})$. Later, $1 \mathrm{~mL} 3 \mathrm{wt} \%$ aq. PS-750-M was added, and the vial was closed screwcap. Then, the reaction vial was transferred to a pre-heated stir plate at $45^{\circ} \mathrm{C}$ and stirred for 50 minutes. After cooling to room temperature, the reaction vial was opened, and arylboronic acid $(0.275 \mathrm{mmol}), p$-benzoquinone $(108 \mathrm{mg}, 0.375 \mathrm{mmol})$, and styrene $(0.25 \mathrm{mmol})$ were added. The reaction vial was closed with a screwcap and then stirred $(1000 \mathrm{rpm})$ at room temperature. Such six reactions were kept in parallel. Every 2 hours one of the six reactions were analyzed using GCMS after the addition of mesitylene $(35.0 \mu \mathrm{L}, 0.25 \mathrm{mmol}$, internal standard) and $1.0 \mathrm{~mL}$ of EtOAc.

\section{$\underline{4.5}$ Procedure-Stirring rate $@ 1500 \mathrm{rpm}$}

A $4.0 \mathrm{~mL}$ reaction vial containing a PTFE-coated magnetic stir bar was charged with nanoparticle precursor palladium(II)acetate $(1.7 \mathrm{mg}, 0.0075 \mathrm{mmol})$. Later, $1 \mathrm{~mL} 3 \mathrm{wt} \%$ aq. PS-750-M was added, and the vial was closed screwcap. Then, the reaction vial was transferred to a pre-heated stir plate at $45^{\circ} \mathrm{C}$ and stirred for 50 minutes. After cooling to room temperature, the reaction vial was opened, and arylboronic acid $(0.275 \mathrm{mmol}), p$-benzoquinone $(108 \mathrm{mg}, 0.375 \mathrm{mmol})$, and styrene $(0.25 \mathrm{mmol})$ were added. The reaction vial was closed with a screwcap and then stirred $(1500 \mathrm{rpm})$ at room temperature. Such six reactions were kept in parallel. Every 2 hours one of the six reactions were analyzed using GCMS after the addition of mesitylene $(35.0 \mu \mathrm{L}, 0.25 \mathrm{mmol}$, internal standard) and $1.0 \mathrm{~mL}$ of EtOAc.

The reaction kinetics varied significantly based on the rate of stirring, this can be accounted based on the nanoparticle generation and proper mixing of the heterogenous mixture. The obtained results are summarized in the Figure S7. 


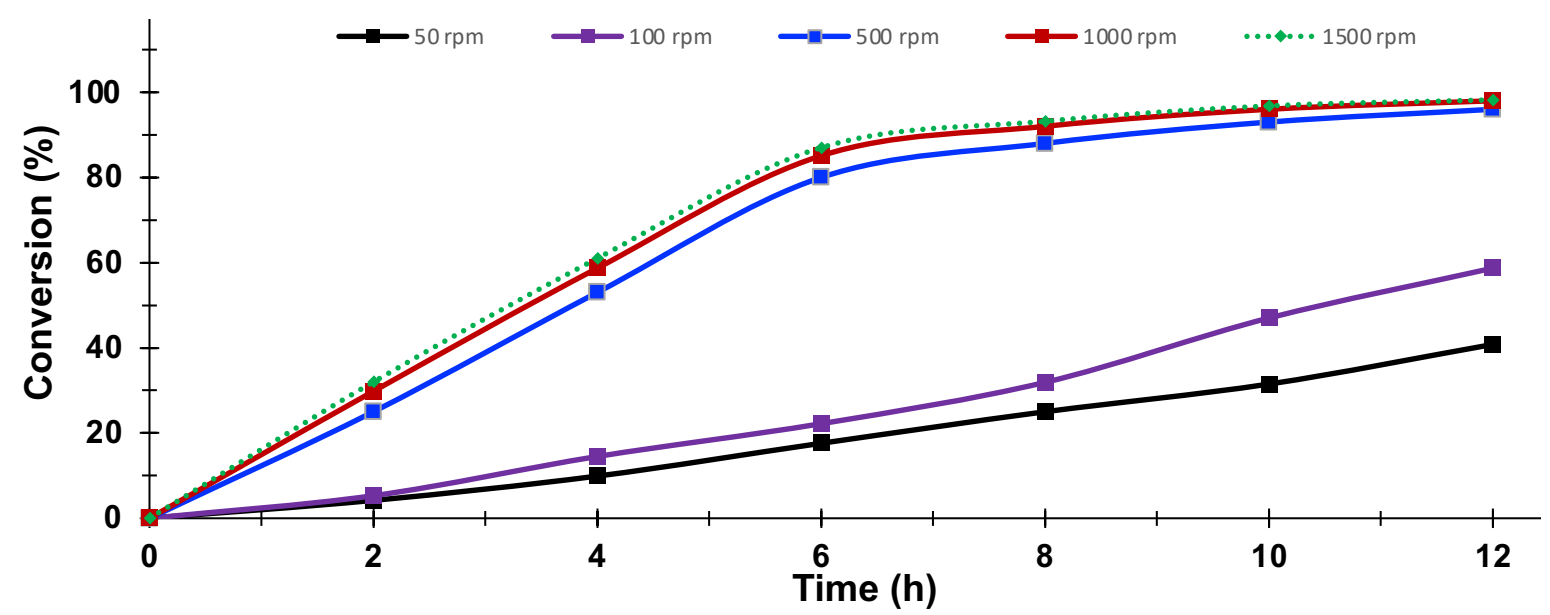

Figure S7. Effect of stirring rate on reaction outcome. 


\section{FILTRATION \& EXTRACTION TEST}

\subsection{Filtration Test}

To study the effect of plausible leaching of nanoparticles, kinetic experiments were performed after filtering the NPs solution through glass fiber filter, keeping the 4-trifluoromethyl phenylboronic acid (4) and styrene (5) as benchmark substrates under standard conditions.

In a $10.0 \mathrm{~mL}$ round-bottom flask containing a PTFE-coated magnetic stir bar, palladium(II)acetate (10.2 $\mathrm{mg}, 0.0454 \mathrm{mmol}$ ) was added. Later, $6.0 \mathrm{~mL} 3 \mathrm{wt} \%$ aq. PS-750-M was added to the reaction mixture. The round-bottom flask was closed with a rubber septum and stirred at $45{ }^{\circ} \mathrm{C}$ for 50 minutes. Later, the obtained nanoparticle suspension was stirred again at $65{ }^{\circ} \mathrm{C}$ for additional $2 \mathrm{~h}$ to ensure the generation of ultrasmall Pd NPs, later the container was allowed to cool to room temperature, and the obtained dark solution was filtered through a filter pipet containing glass fiber filter. Then, the filtrate was transferred to six $4.0 \mathrm{~mL}$ reaction vials ( $1 \mathrm{~mL}$ each) containing PTFEcoated magnetic stir bar, arylboronic acid $(0.275 \mathrm{mmol}), p$-benzoquinone $(0.375 \mathrm{mmol})$ and styrene $(0.25 \mathrm{mmol})$. The reaction vials were closed with screwcaps and then stirred $(1000 \mathrm{rpm})$ at room temperature. Every 2 hours one of the six reactions were analyzed using GCMS after the addition of mesitylene (35.0 $\mu \mathrm{L}, 0.25 \mathrm{mmol}$, internal standard) and $1.0 \mathrm{~mL}$ of EtOAc.

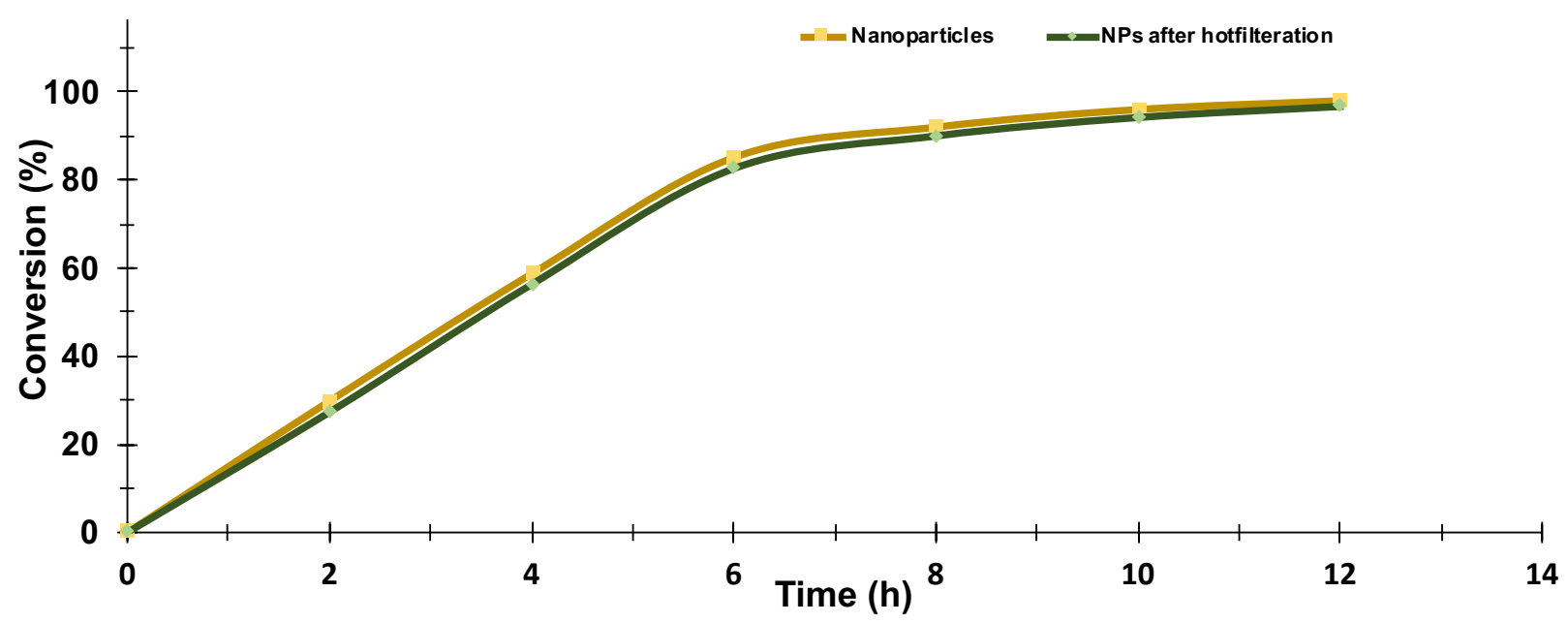

Figure S8. Filtration study.

\section{$\underline{5.2 \text { Extraction test }}$}

To study the effect of plausible leaching of nanoparticles, kinetic experiments were performed after extracting the NPs solution with warm toluene, keeping the 4-trifluoromethyl phenylboronic acid (4) and styrene (5) as benchmark substrates under standard conditions. 
In a $10.0 \mathrm{~mL}$ round-bottom flask containing a PTFE-coated magnetic stir bar, palladium(II)acetate (10.2 $\mathrm{mg}, 0.0454 \mathrm{mmol}$ ) was added. Later, $6.0 \mathrm{~mL} 3 \mathrm{wt} \%$ aq. PS-750-M was added to the reaction mixture. The round-bottom flask was closed with a rubber septum and stirred at $45{ }^{\circ} \mathrm{C}$ for 50 minutes. Later, the container was allowed to cool to room temperature, and the obtained dark solution was transferred to large test tube. Then, $2.0 \mathrm{~mL}$ warm $\left(65^{\circ} \mathrm{C}\right)$ toluene $(2 \mathrm{~mL})$ was added and stirred for additional $2 \mathrm{~h}$, and reactions were performed with both the organic and aqueous layer. The reaction in toluene was performed under standard conditions (arylboronic acid (0.275 $\mathrm{mmol}), p$-benzoquinone $(0.375 \mathrm{mmol})$, styrene $(0.25 \mathrm{mmol})$ and $1.0 \mathrm{~mL}$ toluene extract), traces of product formation was observed $(<1 \%)$ using GCMS analysis of the reaction mixture after $12 \mathrm{~h}$ using mesitylene (1 equiv.) as internal standard.

Then, the filtrate was transferred to six $4.0 \mathrm{~mL}$ reaction vials $(1 \mathrm{~mL}$ each) containing PTFE-coated magnetic stir bar, arylboronic acid $(0.275 \mathrm{mmol}), p$-benzoquinone $(0.375 \mathrm{mmol})$ and styrene $(0.25$ $\mathrm{mmol})$. The reaction vials were closed with screwcaps and stirred $(1000 \mathrm{rpm})$ at room temperature. Every 2 hours one of the six reactions were analyzed using GCMS after the addition of mesitylene $(35.0 \mu \mathrm{L}, 0.25 \mathrm{mmol})$ and $1.0 \mathrm{~mL}$ of EtOAc.

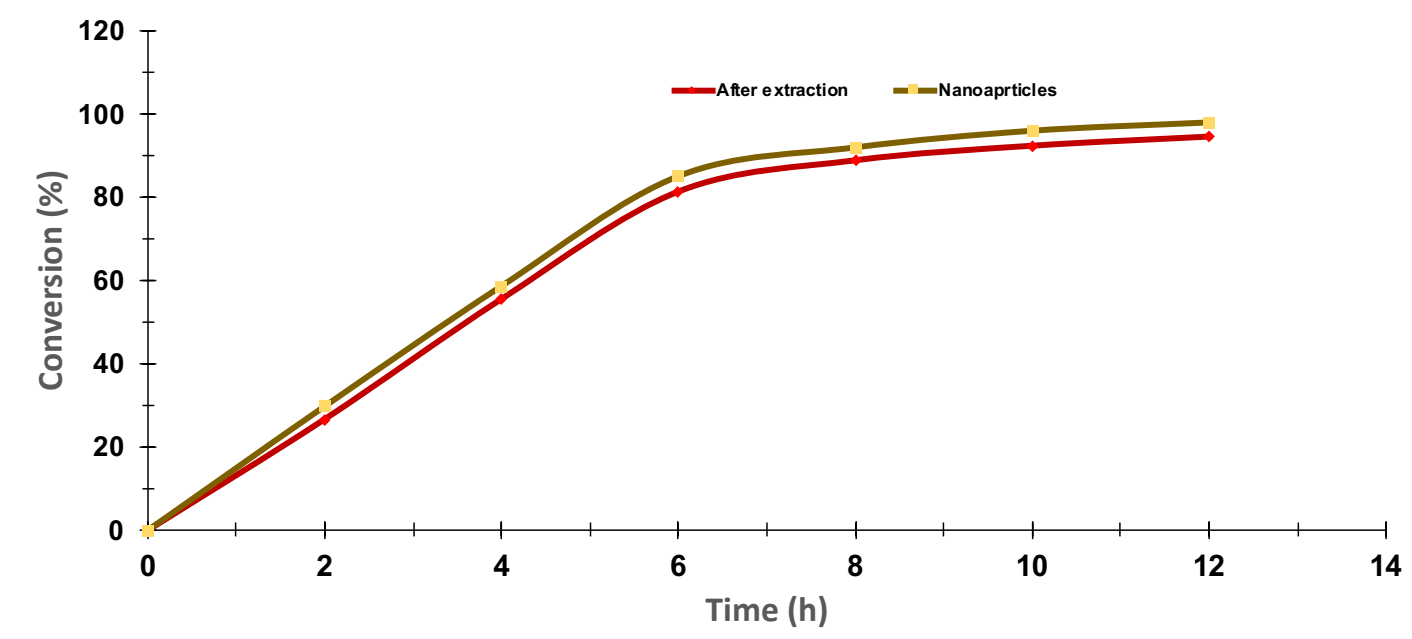

Figure S9. Kinetic studies after extraction. 


\section{THREE-PHASE TEST}

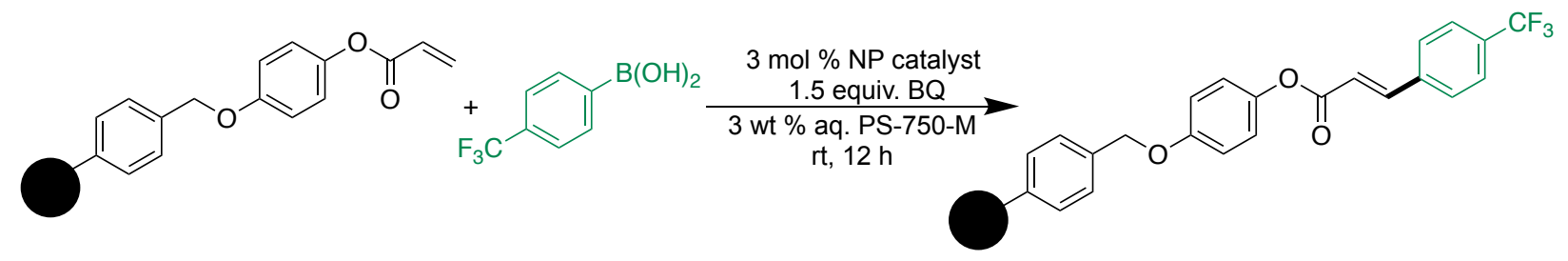

Scheme S1. Three-phase test

A $4.0 \mathrm{~mL}$ reaction vial containing a PTFE-coated magnetic stir bar was charged with nanoparticle precursor palladium(II)acetate $(1.7 \mathrm{mg}, 0.0075 \mathrm{mmol})$. Later, $1 \mathrm{~mL} 3 \mathrm{wt} \%$ aq. PS-750-M was added, and the vial was closed screwcap. Then, the reaction vial was transferred to a pre-heated stir plate at $45^{\circ} \mathrm{C}$ and stirred for 50 minutes. After cooling to room temperature, the reaction vial was opened, and aryl boronic acid (52.8 $\mathrm{mg}, 0.275 \mathrm{mmol}$ ), $p$-benzoquinone (40.5 $\mathrm{mg}, 0.375$ $\mathrm{mmol})$, and REM resin $(278 \mathrm{mg}, 0.25 \mathrm{mmol})$ were added. The reaction vial was closed with a screwcap and then stirred $(1500 \mathrm{rpm})$ at room temperature. After $12 \mathrm{~h}$, the reaction was monitored using TLC, most of the boronic acid remained unreacted. The remaining solid ball was filtered and washed with water, and ethyl acetate. Further, the resin removal was attempted by hydrogenolysis using $\mathrm{Pd} / \mathrm{C}$, where this material remains unreacted. 


\section{REACTION OPTIMIZATION}

Table S3. Screening of nanoparticle precursors ${ }^{\mathrm{a}}$

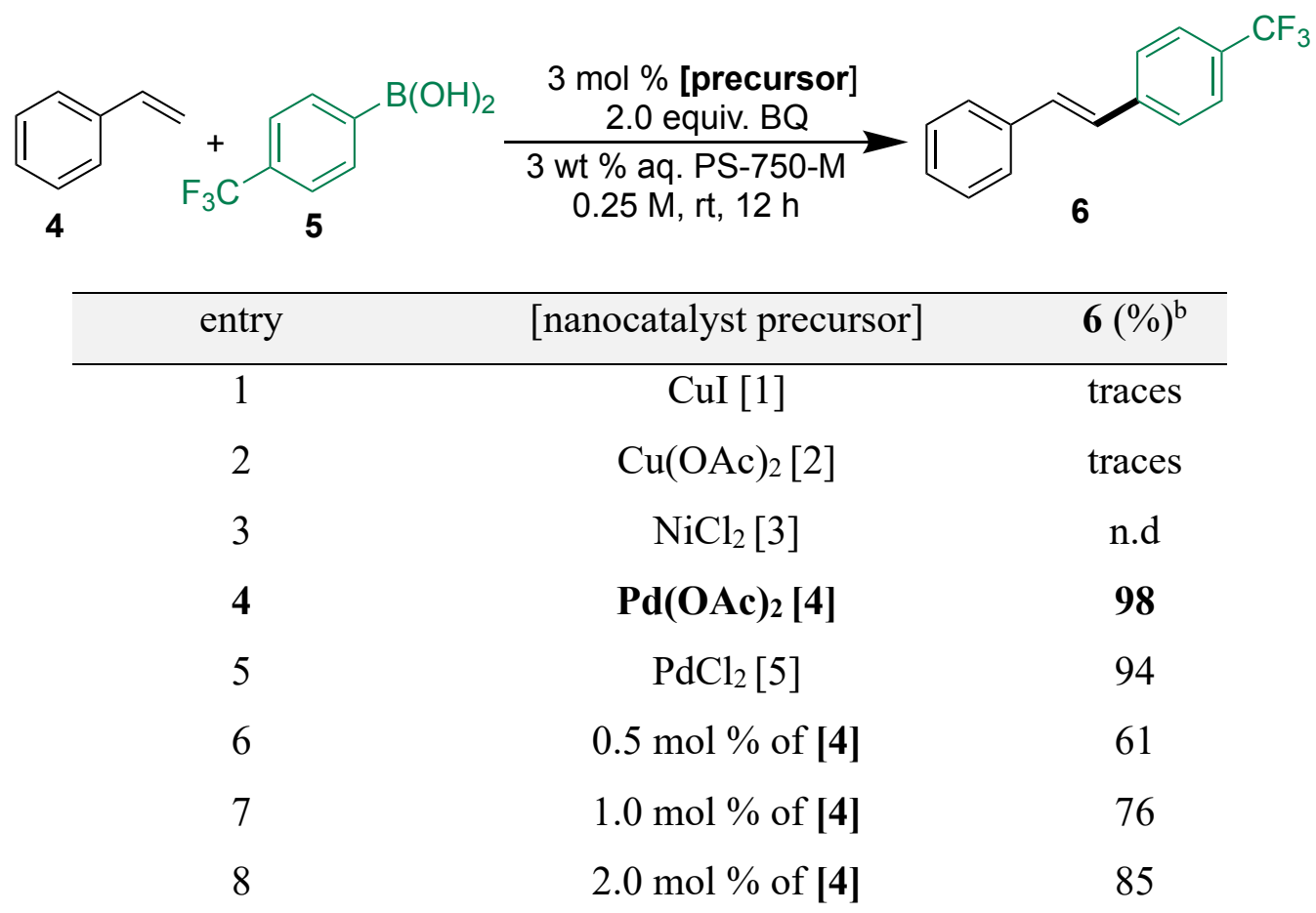

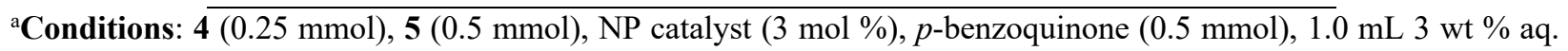
PS-750-M, $23{ }^{\circ} \mathrm{C}, 12 \mathrm{~h}, 1500 \mathrm{rpm}$; ${ }^{b}$ conversions based on GCMS using mesitylene $(0.25 \mathrm{mmol})$ as internal standard. All nanocatalysts were prepared according to procedure described in section 2.

Table S4. Oxidant screening ${ }^{\mathrm{a}}$

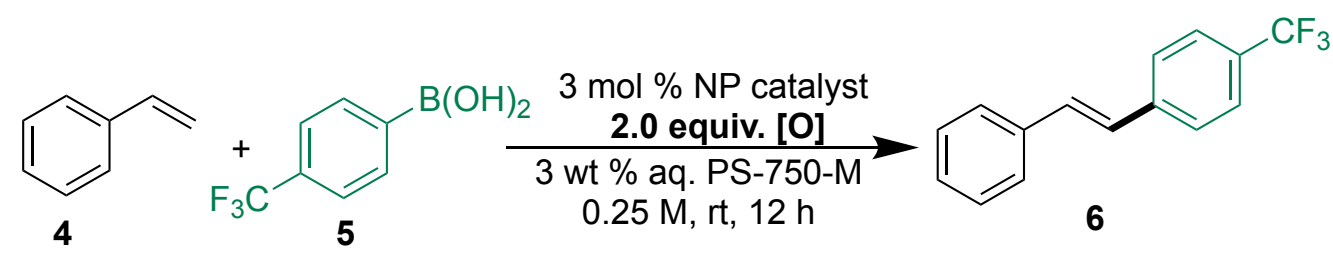

\begin{tabular}{ccc}
\hline entry & oxidant & $\mathbf{6}(\%)^{\mathrm{b}}$ \\
\hline 1 & air & 14 \\
2 & air balloon & 70 \\
3 & Oxygen balloon & 63 \\
$\mathbf{4}$ & $\boldsymbol{p}$-benzoquinone & $\mathbf{9 8}$
\end{tabular}

${ }^{a}$ Conditions: 4 (0.25 mmol), 5 (0.5 mmol), NP catalyst (3 mol \%), oxidant $(0.5 \mathrm{mmol}), 1.0 \mathrm{~mL} 3 \mathrm{wt} \%$ aq. PS-750$\mathrm{M}, 23{ }^{\circ} \mathrm{C}, 12 \mathrm{~h}, 1500 \mathrm{rpm} ;{ }^{b}$ conversions based on GCMS using mesitylene $(0.25 \mathrm{mmol})$ as internal standard. 
Table S5. Oxidant equivalence ${ }^{\mathrm{a}}$

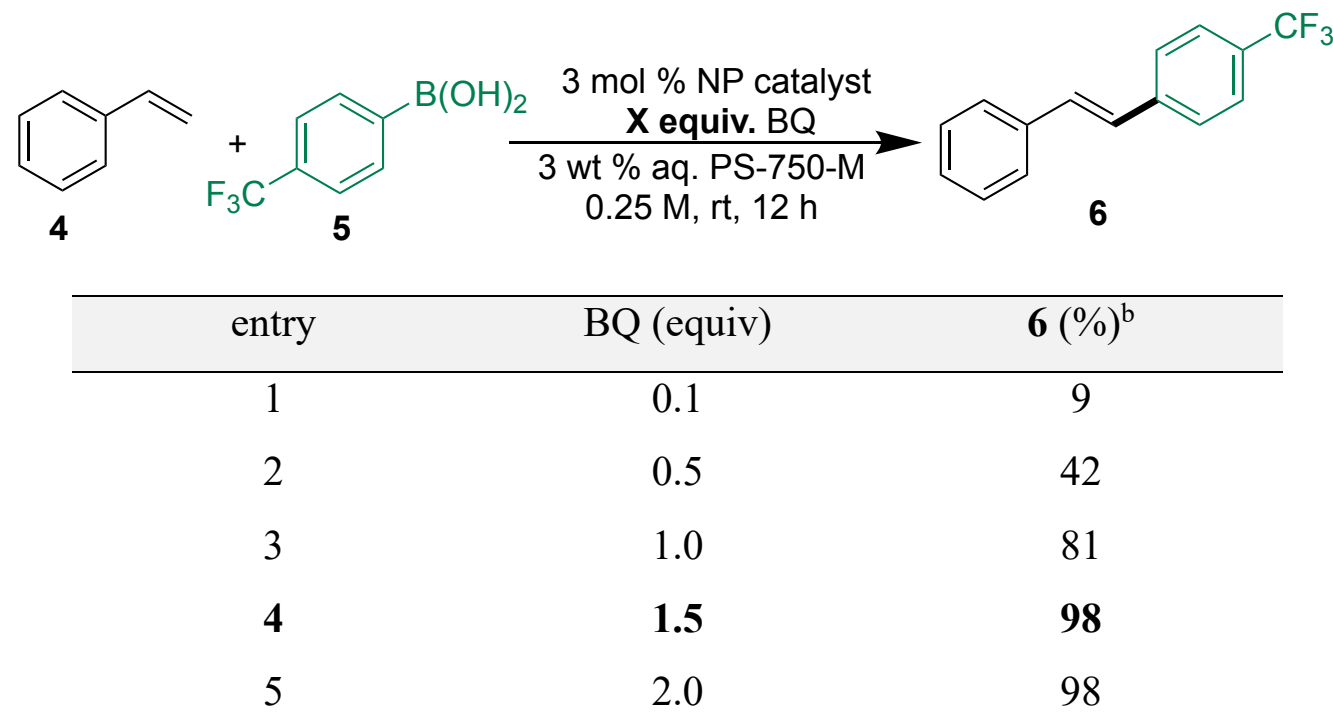

${ }^{a}$ Conditions: $4(0.25 \mathrm{mmol}), 5(0.5 \mathrm{mmol})$, NP catalyst (3 mol \%), p-benzoquinone (X mmol), $1.0 \mathrm{~mL} 3 \mathrm{wt} \%$ aq. PS$750-\mathrm{M}, 23{ }^{\circ} \mathrm{C}, 12 \mathrm{~h}, 1500 \mathrm{rpm} ;{ }^{b}$ conversions based on GCMS using mesitylene $(0.25 \mathrm{mmol})$ as internal standard.

Table S6. Boronic acid loading

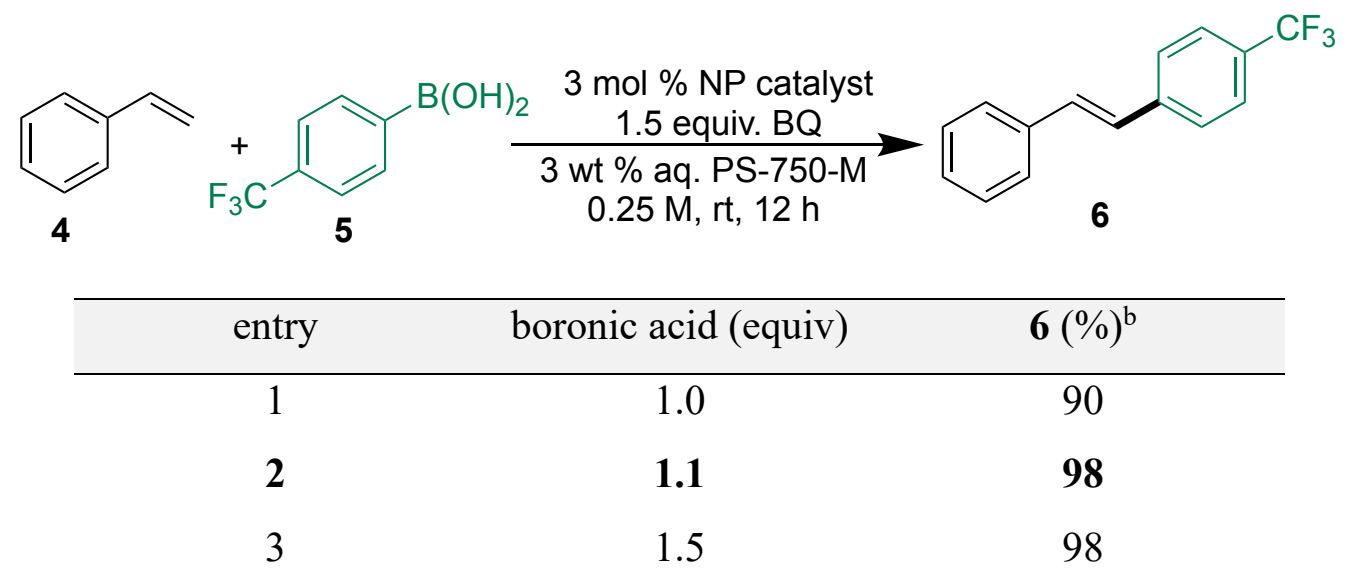

${ }^{\mathrm{a}}$ Conditions: 4 (0.25 mmol), 5 (X mmol), NP catalyst (3 mol \%), p-benzoquinone $(0.375 \mathrm{mmol}), 1.0 \mathrm{~mL} 3 \mathrm{wt} \%$ aq. PS-750-M, $23{ }^{\circ} \mathrm{C}, 12 \mathrm{~h}, 1500 \mathrm{rpm} ;{ }^{b}$ conversions based on GCMS using mesitylene $(0.25 \mathrm{mmol})$ as internal standard. 
Table S7. Global concentration optimization ${ }^{\mathrm{a}}$

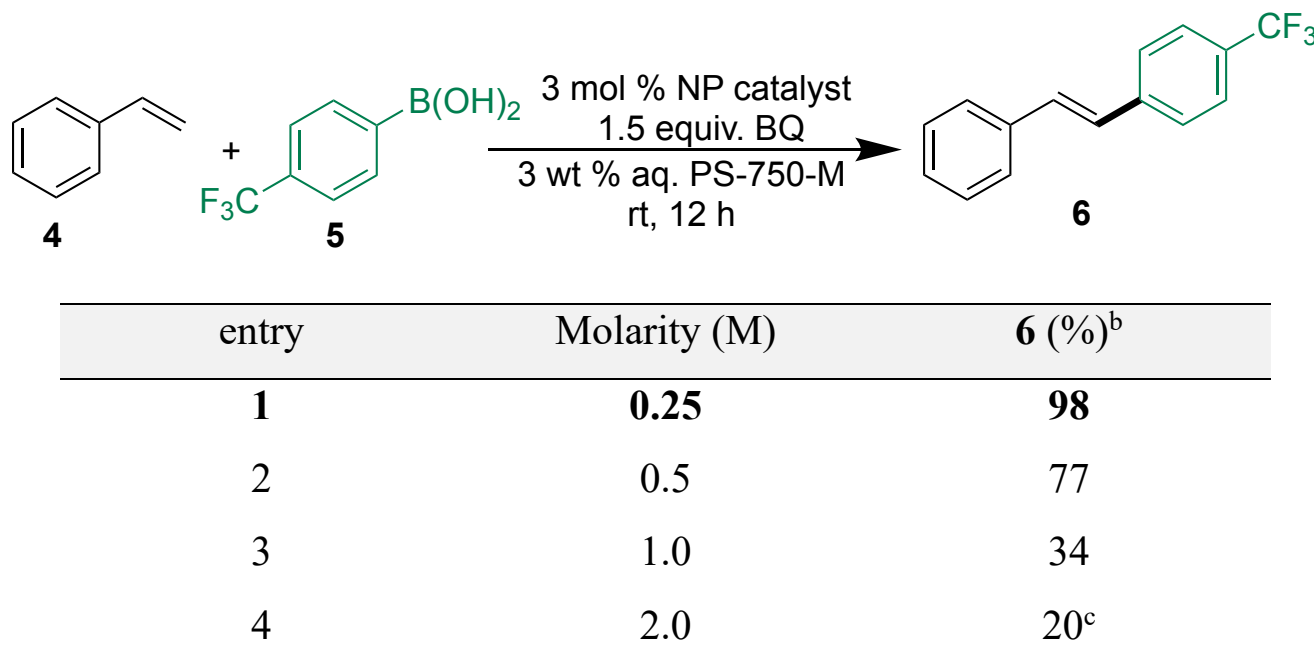

${ }^{a}$ Conditions: $4(0.25 \mathrm{mmol}), 5(0.275 \mathrm{mmol})$, NP catalyst (3 mol \%), p-benzoquinone $(0.375 \mathrm{mmol}), 1 \mathrm{~mL} 3 \mathrm{wt} \%$ aq. PS-750-M, $23{ }^{\circ} \mathrm{C}, 12 \mathrm{~h}, 1500 \mathrm{rpm} ;{ }^{b}$ conversions based on GCMS using mesitylene $(0.25 \mathrm{mmol})$ as internal standard; cthick reaction mass, additional $1 \mathrm{~mL}$ of EtOAc was added during the work-up.

Table S8. Screening reaction solvents ${ }^{\mathrm{a}}$

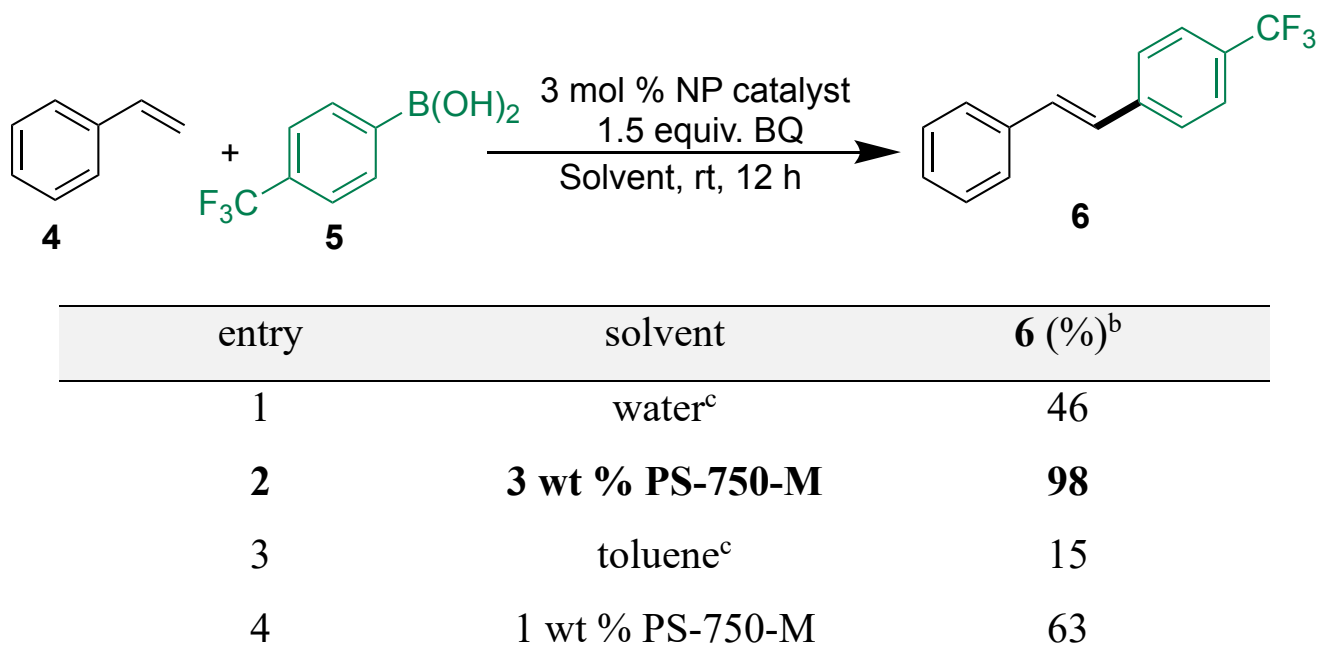

${ }^{a}$ Conditions: $4 \overline{(0.25 \mathrm{mmol}), 5(0.275 \mathrm{mmol}) \text {, NP catalyst (3 mol \%), } p \text {-benzoquinone }(0.375 \mathrm{mmol}), 1} \mathrm{~mL}$ of solvent, $23{ }^{\circ} \mathrm{C}, 12 \mathrm{~h}, 1500 \mathrm{rpm} ;{ }^{b}$ conversions based on GCMS using mesitylene $(0.25 \mathrm{mmol})$ as internal standard. ${ }^{c}$ isolated yield. NPs were prepared in aq. PS-750-M. 


\section{OPTIMIZED PROCEDURE FOR CATALYTIC COUPLINGS}

A $4.0 \mathrm{~mL}$ reaction vial containing a PTFE-coated magnetic stir bar was charged with nanoparticle precursor palladium(II)acetate $(1.7 \mathrm{mg}, 0.0075 \mathrm{mmol})$. Later, $1 \mathrm{~mL} 3 \mathrm{wt} \%$ aq. PS-750-M was added, and the vial was closed screwcap. Then, the reaction vial was transferred to a pre-heated stir plate at $45{ }^{\circ} \mathrm{C}$ and stirred for 50 minutes. After cooling to room temperature, the reaction vial was opened, and arylboronic acid $(0.275 \mathrm{mmol}), p$-benzoquinone $(108 \mathrm{mg}, 0.375 \mathrm{mmol})$, and styrene $(0.25 \mathrm{mmol})$ were added. The reaction vial was closed with a screwcap and then stirred $(1500 \mathrm{rpm})$ at room temperature. After complete consumption of styrene as monitored by TLC or GCMS, the reaction vial was opened, and 1-2 $\mathrm{mL}$ ethyl acetate was added. The mixture was then gently stirred for $2 \mathrm{~min}$ at $\mathrm{rt}$. Stirring was stopped, and the organic layer was separated using a pipette. This extraction was repeated for one more time. The combined organic layers were passed through a celite-pad filled in the pipette. Volatiles were evaporated under reduced pressure to obtain the crude product, which was then purified by flash chromatography over silica gel using hexanes/ethyl acetate as eluent. 


\section{SUBSTRATE SCOPE}

Table S9. Substrate scope

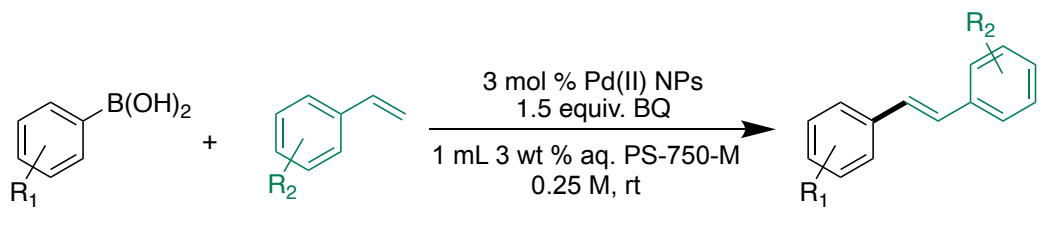

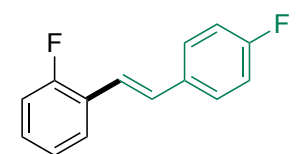

$390 \%, 12 \mathrm{~h}$

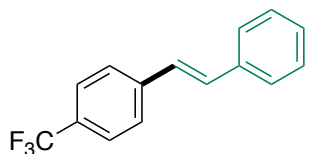

$695 \%, 12 \mathrm{~h}$

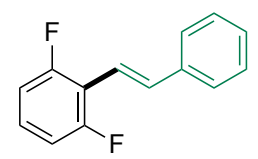

$776 \%, 10 \mathrm{~h}$<smiles>FC(F)(F)c1ccc(C=CCCc2ccccc2)cc1</smiles>

$978 \%, 14 \mathrm{~h}$<smiles>O=[N+]([O-])c1ccc(/C=C/c2ccccc2F)cc1</smiles>

$1080 \%, 10 \mathrm{~h}$

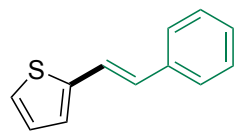

$1177 \%, 16 \mathrm{~h}$

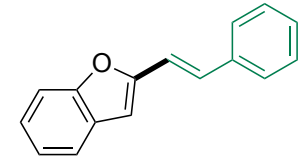

$872 \%, 11 \mathrm{~h}$<smiles>COc1ccc(/C=C/c2ccccc2)cc1</smiles>

$1371 \%, 18 \mathrm{~h}$<smiles>Fc1c(F)c(F)c(/C=C/c2ccccc2C(F)(F)F)c(F)c1F</smiles>

$1490 \%, 7 \mathrm{~h}$

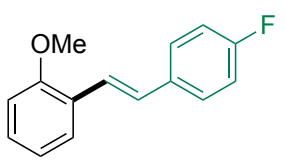

$1583 \%, 11 \mathrm{~h}$

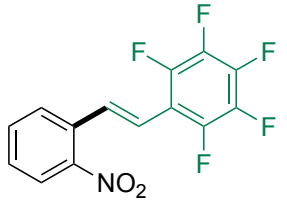

$1280 \%, 10 \mathrm{~h}$<smiles>O=[N+]([O-])c1ccc(/C=C/c2c(F)c(F)c(F)c(F)c2F)cc1</smiles>

$1781 \%, 12 \mathrm{~h}$<smiles>Fc1ccccc1/C=C/c1ccccc1</smiles>

$1889 \%, 12 \mathrm{~h}$

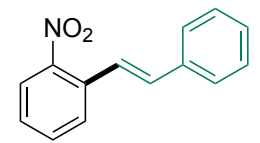

$1994 \%, 12 \mathrm{~h}$<smiles>Fc1ccccc1/C=C/c1c(F)c(F)c(F)c(F)c1F</smiles>

$1685 \%, 7 \mathrm{~h}$<smiles>Fc1cccc(F)c1/C=C/c1c(F)c(F)c(F)c(F)c1F</smiles>

$2082 \%, 7 \mathrm{~h}$

$2291 \%, 8 \mathrm{~h}$<smiles>COc1ccccc1/C=C/c1c(F)c(F)c(F)c(F)c1F</smiles>

$2384 \%, 12 \mathrm{~h}$<smiles>Fc1c(F)c(F)c(/C=C/c2cc3ccccc3o2)c(F)c1F</smiles>

$2465 \%, 11 \mathrm{~h}$<smiles>Cc1ccc(/C=C/c2ccccc2)c([N+](=O)[O-])c1</smiles>

$2573 \%, 16 \mathrm{~h}$<smiles>COc1ccc(/C=C/c2ccc(C(F)(F)F)cc2)cc1</smiles>

$2681 \%, 20 \mathrm{~h}$<smiles>C(=C/c1cccc2ccccc12)\c1ccccc1</smiles>

$2766 \%, 19 \mathrm{~h}$<smiles>Cc1ccc(/C=C/c2ccc(C(F)(F)F)cc2)cc1</smiles>

$2885 \%, 18 \mathrm{~h}$<smiles>COC(=O)/C=C/c1ccc(C(F)(F)F)cc1</smiles><smiles>COC(=O)/C=C/c1c(F)cccc1F</smiles><smiles>COC(=O)/C=C/c1ccccc1[N+](=O)[O-]</smiles><smiles>CCCOC(=O)/C=C/c1ccc(C(F)(F)F)cc1</smiles>

$2980 \%, 15 \mathrm{~h}$

$3070 \%, 15 \mathrm{~h}$

$3176 \%, 15 \mathrm{~h}$

${ }^{a}$ Conditions: styrene $(0.25 \mathrm{mmol})$, arylboronic acid $(0.275 \mathrm{mmol})$, NP catalyst $(3 \mathrm{~mol} \%)$, $p$-benzoquinone $(0.375$ $\mathrm{mmol}), 1 \mathrm{~mL}$ of $3 \mathrm{wt} \%$ aq. PS-750-M, $23^{\circ} \mathrm{C}$. 


\section{MULTI-GRAM SCALE REACTION}

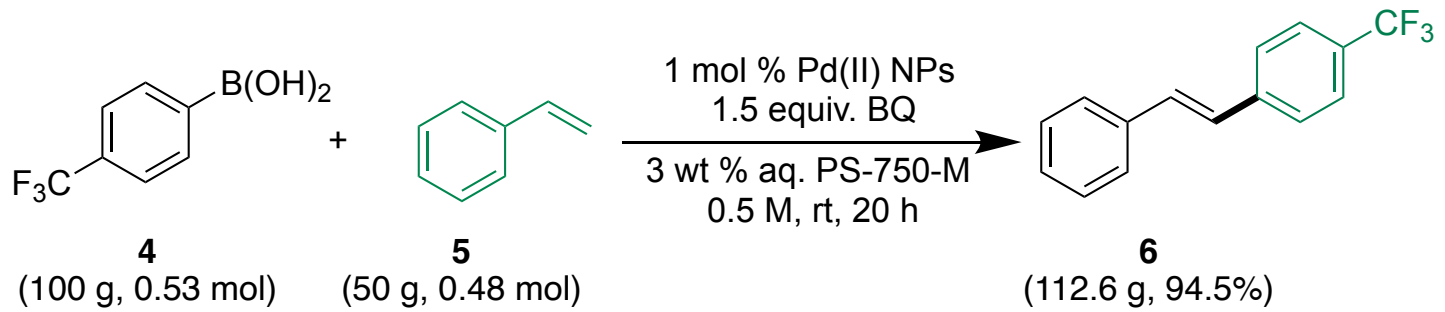

Scheme S2. Multi-gram scale reaction

In a 2.0 L round-bottom flask containing a PTFE-coated magnetic stir bar, 4trifluoromethylphenylboronic acid (100 g, $0.53 \mathrm{~mol})$, -benzoquinone (85.9 g, $0.79 \mathrm{~mol})$, nanocatalyst precursor $(1.19 \mathrm{~g}, 0.0053 \mathrm{~mol})$ and styrene $(50 \mathrm{~g}, 0.48 \mathrm{~mol})$ were added. Later, 960 $\mathrm{mL} 3 \mathrm{wt} \%$ aq. PS-750-M was added to the reaction mixture. The round-bottom flask was closed with a rubber septum, and the reaction mixture was stirred $(1500 \mathrm{rpm})$ at room temperature for 20 h. After complete consumption of styrene as monitored by TLC or GCMS, rubber septum was removed. $50 \mathrm{~mL} 3 \mathrm{~N}$ aq. $\mathrm{NaOH}$ was added and stirred at $45^{\circ} \mathrm{C}$ for 30 minutes. Later the reaction mixture was transferred to a $2.0 \mathrm{~L}$ separatory funnel, $100 \mathrm{~mL}$ hexanes was added and extracted. The extraction procedure was repeated at least three times (TLC was checked on each fraction to ensure the complete extraction). The combined organic layers were passed through celite-pad. Volatiles were removed under reduced pressure to obtain pure product as white solid $\left(R_{\mathrm{f}} 0.53\right.$, hexanes); yield $112.6 \mathrm{~g}(94.5 \%)$. 


\section{ANALYTICAL DATA}

(E)-1-fluoro-2-(4-fluorostyryl)benzene (3)

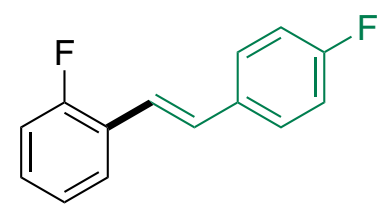

White solid, $48.6 \mathrm{mg}(90 \%), R_{\mathrm{f}} 0.5$ (1:19, EtOAc:hexanes). ${ }^{1} \mathrm{H}$ NMR (400 MHz, $\left.\mathrm{CDCl}_{3}\right): \delta 7.59$ $(\mathrm{t}, J=8 \mathrm{~Hz}, 1 \mathrm{H}), 7.52-7.49(\mathrm{~m}, 2 \mathrm{H}), 7.24-7.21(\mathrm{~m}, 1 \mathrm{H}), 7.17-7.04(\mathrm{~m}, 6 \mathrm{H}) ;{ }^{19} \mathrm{~F}$ NMR $(376 \mathrm{~Hz}$, $\left.\mathrm{CDCl}_{3}\right)-113.8(1 \mathrm{~F}),-118.7(1 \mathrm{~F})$.

(E)-1-styryl-4-(trifluoromethyl)benzene (6) ${ }^{5}$

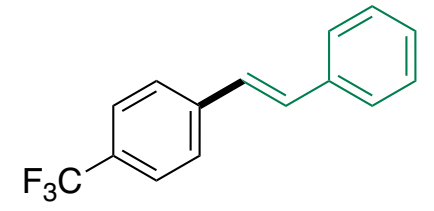

White solid, $59 \mathrm{mg}(95 \%), R_{\mathrm{f}} 0.53$ (hexanes). ${ }^{1} \mathrm{H} \mathrm{NMR}\left(400 \mathrm{MHz}, \mathrm{CDCl}_{3}\right): \delta$ 7.64-7.59 (m, 4H), $7.55(\mathrm{~d}, J=8 \mathrm{~Hz}, 2 \mathrm{H}), 7.40$ (t, $J=8 \mathrm{~Hz}, 2 \mathrm{H}), 7.32$ (t, $J=8 \mathrm{~Hz}, 1 \mathrm{H}), 7.21(\mathrm{~d}, J=16 \mathrm{~Hz}, 1 \mathrm{H}), 7.13$ $(\mathrm{d}, J=16 \mathrm{~Hz}, 1 \mathrm{H}) ;{ }^{19} \mathrm{~F}$ NMR $\left(376 \mathrm{~Hz}, \mathrm{CDCl}_{3}\right)-62.43(3 \mathrm{~F})$.

\section{(E)-1,3-difluoro-2-styrylbenzene (7) ${ }^{6}$}

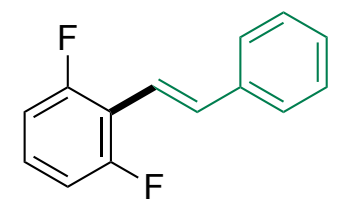

White solid, $41 \mathrm{mg}(76 \%), R_{\mathrm{f}} 0.32$ (hexanes). ${ }^{1} \mathrm{H} \mathrm{NMR}\left(400 \mathrm{MHz}, \mathrm{CDCl}_{3}\right): \delta 7.53(\mathrm{~d}, J=8 \mathrm{~Hz}$, 2H), $7.44(\mathrm{~d}, J=16 \mathrm{~Hz}, 1 \mathrm{H}), 7.38-7.35(\mathrm{~m}, 2 \mathrm{H}), 7.30-7.25(\mathrm{~m}, 1 \mathrm{H}), 7.17-7.12(\mathrm{~m}, 1 \mathrm{H}), 7.13(\mathrm{~d}, J$ $=16 \mathrm{~Hz}, 1 \mathrm{H}), 6.90(\mathrm{t}, J=8 \mathrm{~Hz}, 2 \mathrm{H}) ;{ }^{19} \mathrm{~F} \mathrm{NMR}\left(376 \mathrm{MHz}, \mathrm{CDCl}_{3}\right):-113.00(2 \mathrm{~F})$. 


\section{(E)-2-styrylbenzofuran $(8)^{7}$}

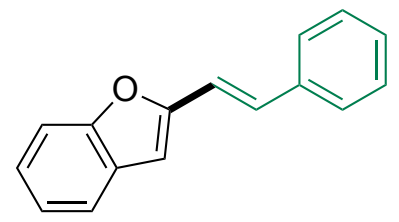

White solid, $39.6 \mathrm{mg}$ (72\%), $R_{\mathrm{f}} 0.39$ (1:19, EtOAc:hexanes). ${ }^{1} \mathrm{H}$ NMR (500 MHz, $\left.\mathrm{CDCl}_{3}\right): \delta 7.55$ $(\mathrm{d}, J=5 \mathrm{~Hz}, 3 \mathrm{H}), 7.49-7.51(\mathrm{~m}, 1 \mathrm{H}), 7.42-7.39(\mathrm{~m}, 2 \mathrm{H}), 7.33-7.29(\mathrm{~m}, 3 \mathrm{H}), 7.25-7.21(\mathrm{~m}, 1 \mathrm{H})$, $7.03(\mathrm{~d}, J=15 \mathrm{~Hz}, 1 \mathrm{H}), 6.70(\mathrm{~s}, 1 \mathrm{H})$.

\section{(E)-1-(4-phenylbut-1-en-1-yl)-4-(trifluoromethyl)benzene (9) ${ }^{8}$}

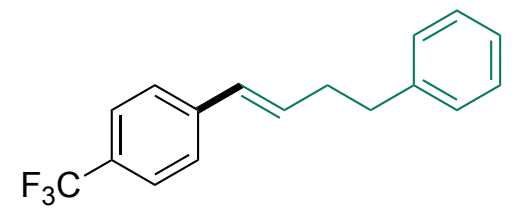

Waxy solid, $53.8 \mathrm{mg}(78 \%), R_{\mathrm{f}} 0.29$ (hexanes). ${ }^{1} \mathrm{H}$ NMR $\left(400 \mathrm{MHz}, \mathrm{CDCl}_{3}\right): \delta 7.52(\mathrm{~d}, J=8 \mathrm{~Hz}$, 2H), 7.39 (d, $J=8 \mathrm{~Hz}, 1 \mathrm{H}), 7.30-7.27$ (m, 3H), 7.20 (d, $J=8 \mathrm{~Hz}, 3 \mathrm{H}), 6.44-6.34$ (m, 2H), 2.84$2.77(\mathrm{~m}, 2 \mathrm{H}), 2.58-2.52(\mathrm{~m}, 2 \mathrm{H}) ;\left(376 \mathrm{~Hz}, \mathrm{CDCl}_{3}\right)-62.46(3 \mathrm{~F})$.

\section{(E)-1-fluoro-2-(4-nitrostyryl)benzene (10)9}

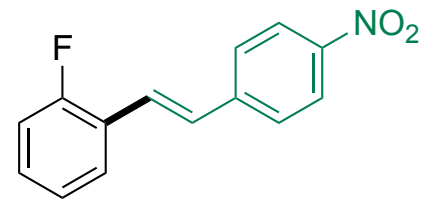

Yellow waxy liquid, $48.6 \mathrm{mg}(80 \%), R_{\mathrm{f}} 0.41$ (1:4 EtOAc:hexanes). ${ }^{1} \mathrm{H}$ NMR (400 MHz, $\left.\mathrm{CDCl}_{3}\right)$ : $\delta 8.21$ (dd, $J=4 \mathrm{~Hz}, 8 \mathrm{~Hz}, 2 \mathrm{H}), 7.65-7.60$ (m, 3H), 7.41 (d, $J=16 \mathrm{~Hz}, 1 \mathrm{H}), 7.36-7.28$ (m, 1H), 7.23-7.08 (m, 3H); ${ }^{19} \mathrm{~F}$ NMR $\left(376 \mathrm{~Hz}, \mathrm{CDCl}_{3}\right)-116.87(1 \mathrm{~F})$. 
(E)-2-styrylthiophene (11) ${ }^{10}$

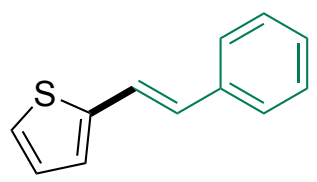

Light yellow liquid, $35.8 \mathrm{mg}(77 \%), R_{\mathrm{f}} 0.36$ (1:9 hexanes). ${ }^{1} \mathrm{H}$ NMR (500 MHz, $\left.\mathrm{CDCl}_{3}\right): \delta 7.48$ $(\mathrm{d}, J=5 \mathrm{~Hz}, 2 \mathrm{H}), 7.38-7.4(\mathrm{~m}, 2 \mathrm{H}), 7.28-7.21(\mathrm{~m}, 3 \mathrm{H}), 7.09(\mathrm{~s}, 1 \mathrm{H}), 7.03-7.01(\mathrm{~m}, 1 \mathrm{H}), 6.95(\mathrm{~d}, J$ $=15 \mathrm{~Hz}, 1 \mathrm{H})$.

\section{(E)-1,2,3,4,5-pentafluoro-6-(2-nitrostyryl)benzene (12)}

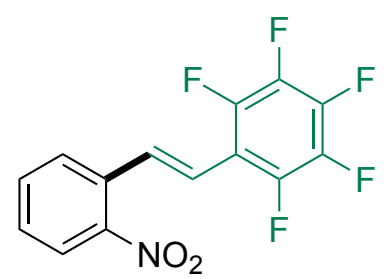

Waxy yellow liquid, $63 \mathrm{mg}(80 \%), R_{\mathrm{f}} 0.28$ (1:9 EtOAc:hexanes). ${ }^{1} \mathrm{H}$ NMR (400 MHz, $\left.\mathrm{CDCl}_{3}\right): \delta$ $8.04(\mathrm{~d}, J=8 \mathrm{~Hz}, 1 \mathrm{H}), 7.91(\mathrm{~d}, J=16 \mathrm{~Hz}, 1 \mathrm{H}), 7.73-7.66(\mathrm{~m}, 2 \mathrm{H}), 7.51(\mathrm{t}, J=8 \mathrm{~Hz}, 1 \mathrm{H}), 6.91(\mathrm{~d}$, $J=16 \mathrm{~Hz}, 1 \mathrm{H}) ;{ }^{13} \mathrm{C} \mathrm{NMR}\left(100 \mathrm{MHz}, \mathrm{CDCl}_{3}\right): \delta 148.1,145.2(\mathrm{~d}, J=250 \mathrm{~Hz}), 140.6(\mathrm{~d}, J=254$ $\mathrm{Hz}), 138.0(\mathrm{~d}, J=251 \mathrm{~Hz}), 133.7,133.0(\mathrm{t}, J=8 \mathrm{~Hz}), 132.8,129.4,128.8,125.1,117.7,111.8 ;{ }^{19} \mathrm{~F}$ NMR $\left(376 \mathrm{~Hz}, \mathrm{CDCl}_{3}\right)$-141.96 (2F), -154.28 (1F), -162.31 (2F). IR ( $\left.v \mathrm{~cm}^{-1}\right)$ 2910, 1913, 1835, 1512. HRMS (EI) $\left[\left(\mathrm{C}_{14} \mathrm{H}_{6} \mathrm{~F}_{5} \mathrm{NO}_{2}\right)^{+}\right]$calcd $=315.0319$, found $\mathrm{m} / \mathrm{z}=315.0313$.

\section{(E)-1-methoxy-4-styrylbenzene (13) $)^{11}$}

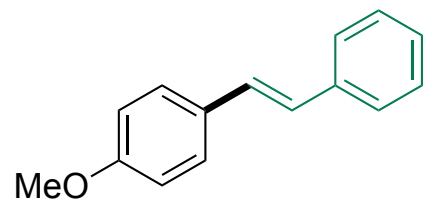

Off-white solid, $37.2 \mathrm{mg}$ (71\%), $R_{\mathrm{f}} 0.53$ (1:19, EtOAc:hexanes). ${ }^{1} \mathrm{H}$ NMR (400 MHz, $\left.\mathrm{CDCl}_{3}\right): \delta$ 7.76-7.70 (m, 4H), $7.60(\mathrm{t}, J=8 \mathrm{~Hz}, 2 \mathrm{H}), 7.50(\mathrm{~d}, J=8 \mathrm{~Hz}, 1 \mathrm{H}), 7.35-7.21(\mathrm{~m}, 2 \mathrm{H}), 7.16(\mathrm{~d}, J=8$ $\mathrm{Hz}, 2 \mathrm{H}), 4.01$ (s, 3H). 


\section{(E)-1,2,3,4,5-pentafluoro-6-(2-(trifluoromethyl)styryl)benzene (14)}

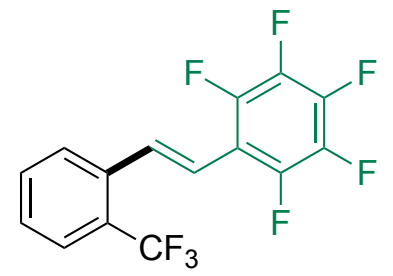

White solid, $\mathrm{mp}=105-106{ }^{\circ} \mathrm{C}, 76 \mathrm{mg}(90 \%), R_{\mathrm{f}} 0.41$ (hexanes). ${ }^{1} \mathrm{H}$ NMR $\left(400 \mathrm{MHz}, \mathrm{CDCl}_{3}\right): \delta$ $7.83(\mathrm{~d}, J=16 \mathrm{~Hz}, 1 \mathrm{H}), 7.76(\mathrm{~d}, J=8 \mathrm{~Hz}, 1 \mathrm{H}), 7.70(\mathrm{~d}, J=8 \mathrm{~Hz}, 1 \mathrm{H}), 7.59$ (t, $J=8 \mathrm{~Hz}, 1 \mathrm{H}), 7.44$ $(\mathrm{t}, J=8 \mathrm{~Hz}, 1 \mathrm{H}), 6.94(\mathrm{~d}, J=16 \mathrm{~Hz}, 1 \mathrm{H}) ;{ }^{13} \mathrm{C} \mathrm{NMR}\left(100 \mathrm{MHz}, \mathrm{CDCl}_{3}\right): \delta 144.9(\mathrm{~d}, J=253 \mathrm{~Hz})$, $140.2(\mathrm{~d}, J=248 \mathrm{~Hz}), 137.7$ (d, $J=248 \mathrm{~Hz}), 135.5,133.1,132.1$, 128.4, 128.0, 127.0, 126.0, 124.1 (q, $273 \mathrm{~Hz}), 116.6,111.8 ;{ }^{19} \mathrm{~F}$ NMR $\left(376 \mathrm{~Hz}, \mathrm{CDCl}_{3}\right)-59.49$ (3F), -142.36 (2F), -155.22 (1F), 162.60 (2F). IR $\left(v \mathrm{~cm}^{-1}\right) 2921,1918,1636,1417$. HRMS $(\mathrm{EI})\left[\left(\mathrm{C}_{15} \mathrm{H}_{6} \mathrm{~F}_{8}\right)^{+}\right]$calcd $=338.0342$, found $\mathrm{m} / \mathrm{z}=338.0341$.

\section{(E)-1-(4-fluorostyryl)-2-methoxybenzene (15) ${ }^{9}$}

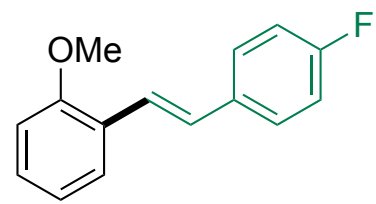

Colorless waxy liquid, $47.3 \mathrm{mg}(83 \%), R_{\mathrm{f}} 0.33$ (1:9, EtOAc:hexanes). ${ }^{1} \mathrm{H}$ NMR (400 MHz, $\left.\mathrm{CDCl}_{3}\right)$ : $\delta 7.56(\mathrm{~d}, J=8 \mathrm{~Hz}, 1 \mathrm{H}), 7.50-7.46(\mathrm{~m}, 2 \mathrm{H}), 7.48(\mathrm{~d}, J=16 \mathrm{~Hz}, 1 \mathrm{H}), 7.25-7.22(\mathrm{~m}, 1 \mathrm{H}), 7.08-7.00$ $(\mathrm{m}, 3 \mathrm{H}), 6.94-6.97(\mathrm{~m}, 1 \mathrm{H}), 6.87(\mathrm{~d}, J=8 \mathrm{~Hz}, 1 \mathrm{H}), 3.88(\mathrm{~s}, 3 \mathrm{H}) ;{ }^{19} \mathrm{~F} \mathrm{NMR}\left(376 \mathrm{~Hz}, \mathrm{CDCl}_{3}\right)$ $114.82(1 \mathrm{~F})$.

\section{(E)-1,2,3,4,5-pentafluoro-6-(2-fluorostyryl)benzene (16)}

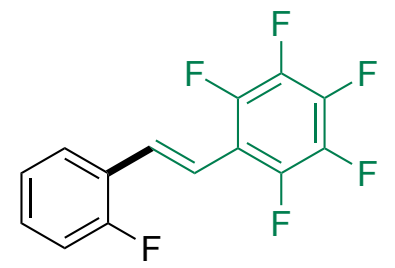

White solid, $\mathrm{mp}=128-129{ }^{\circ} \mathrm{C}, 61.2 \mathrm{mg}(85 \%), R_{\mathrm{f}} 0.29$ (hexanes). ${ }^{1} \mathrm{H}$ NMR $\left(400 \mathrm{MHz}, \mathrm{CDCl}_{3}\right): \delta$ 7.61-7.55 (m, 2H), 7.34-7.28 (m, 1H), 7.16-7.19 (m, 1H), 7.12-7.10 (m, 1H), 7.05 (d, $J=16 \mathrm{~Hz}$, $1 \mathrm{H}) ;{ }^{13} \mathrm{C} \mathrm{NMR}\left(100 \mathrm{MHz}, \mathrm{CDCl}_{3}\right): \delta 160.6(\mathrm{~d}, J=250 \mathrm{~Hz}), 144.8(\mathrm{~d}, J=251 \mathrm{~Hz}), 139.9(\mathrm{~d}, J=$ 
$250 \mathrm{~Hz}), 137.7$ (d, $J=251 \mathrm{~Hz}), 130.2(\mathrm{~d}, J=8.0 \mathrm{~Hz}), 129.7,127.3,124.3,116.0$ (d, $J=22 \mathrm{~Hz})$, 114.9, 112.2; ${ }^{19} \mathrm{~F}$ NMR (376 Hz, $\left.\mathrm{CDCl}_{3}\right)-116.89$ (1F), -142.63 (2F), -156.00 (1F), -162.89 (2F). IR $\left(v \mathrm{~cm}^{-1}\right) 2931,1928,1486$. HRMS (EI) $\left[\left(\mathrm{C}_{14} \mathrm{H}_{6} \mathrm{~F}_{6}\right)^{+}\right]$calcd $=288.0374$, found $\mathrm{m} / \mathrm{z}=288.0371$.

(E)-1,2,3,4,5-pentafluoro-6-(4-nitrostyryl)benzene (17)

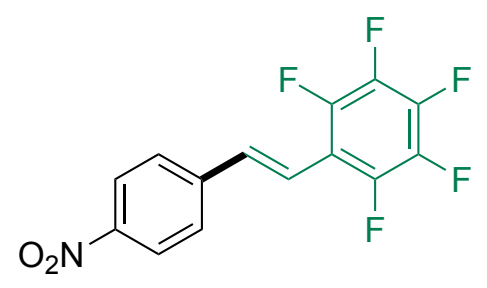

Thick yellow liquid, $64 \mathrm{mg}(81 \%), R_{\mathrm{f}} 0.27$ (1:9, EtOAc:hexanes). ${ }^{1} \mathrm{H}$ NMR (400 MHz, $\left.\mathrm{CDCl}_{3}\right)$ : $\delta$ $8.26(\mathrm{~d}, J=8 \mathrm{~Hz}, 2 \mathrm{H}), 7.68$ (d, $J=8 \mathrm{~Hz}, 2 \mathrm{H}), 7.49$ (d, $J=16 \mathrm{~Hz}, 1 \mathrm{H}), 7.14(\mathrm{~d}, J=16 \mathrm{~Hz}, 1 \mathrm{H})$; ${ }^{13} \mathrm{C}$ NMR (100 MHz, $\left.\mathrm{CDCl}_{3}\right): \delta$ 147.7, 145.1 (d, $\left.J=253 \mathrm{~Hz}\right), 142.7,141.9,137.9(\mathrm{~d}, J=250 \mathrm{~Hz})$, 134.6, 127.5, 124.3, 117.2, 111.5; ${ }^{19} \mathrm{~F}$ NMR (376 Hz, $\left.\mathrm{CDCl}_{3}\right)-141.75$ (2F), -153.97 (1F), -162.08 (2F). IR $\left(v \mathrm{~cm}^{-1}\right) 2920,1905,1430$. HRMS (EI) $\left[\left(\mathrm{C}_{14} \mathrm{H}_{6} \mathrm{~F}_{5} \mathrm{NO}_{2}\right)^{+}\right] \mathrm{calcd}=315.0319$, found $\mathrm{m} / \mathrm{z}=$ 315.0313.

\section{(E)-1-fluoro-2-styrylbenzene $(18)^{12}$}

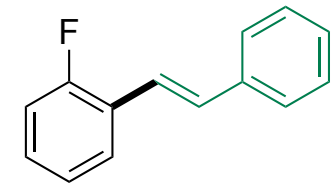

White solid, $44 \mathrm{mg}(89 \%), R_{\mathrm{f}} 0.4$ (hexanes). ${ }^{1} \mathrm{H} \mathrm{NMR}\left(400 \mathrm{MHz}, \mathrm{CDCl}_{3}\right): \delta$ 7.64-7.61 (m, $\left.1 \mathrm{H}\right)$, $7.56(\mathrm{~d}, J=8 \mathrm{~Hz}, 2 \mathrm{H}), 7.41-7.37$ (m, 2H), 7.34-7.22 (m, 4H), 7.15-7.07 (m, 2H); ${ }^{19} \mathrm{~F}$ NMR $(376$ $\left.\mathrm{Hz}, \mathrm{CDCl}_{3}\right)-114.82(1 \mathrm{~F})$. 


\section{(E)-1-nitro-2-styrylbenzene (19) $)^{13}$}

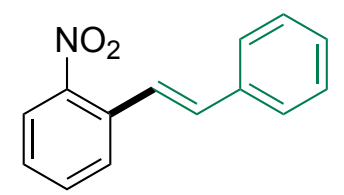

Yellow waxy liquid, $52.8 \mathrm{mg}$ (94\%), $R_{\mathrm{f}} 0.4$ (1:9, EtOAc:hexanes). ${ }^{1} \mathrm{H}$ NMR (400 MHz, $\left.\mathrm{CDCl}_{3}\right)$ : $\delta 7.96(\mathrm{~d}, J=12 \mathrm{~Hz}, 1 \mathrm{H}), 7.77$ (d, $J=8 \mathrm{~Hz}, 1 \mathrm{H}), 7.62-7.54(\mathrm{~m}, 4 \mathrm{H}), 7.42-7.30$ (m, 4H), 7.09 (d, $J$ $=16 \mathrm{~Hz}, 1 \mathrm{H})$.

\section{(E)-1-(2,6-difluorostyryl)-2,3,4,5,6-pentafluorobenzene (20)}

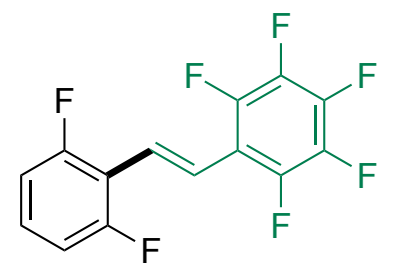

Waxy solid, $62.7 \mathrm{mg}(82 \%), R_{\mathrm{f}} 0.31$ (hexanes). ${ }^{1} \mathrm{H} \mathrm{NMR}\left(500 \mathrm{MHz}, \mathrm{CDCl}_{3}\right): \delta 7.44$ (d, $J=15 \mathrm{~Hz}$, 1H), 7.33-7.23 (m, 2H), 6.95 (t, $J=10 \mathrm{~Hz}, 2 \mathrm{H}) ;{ }^{13} \mathrm{C} \mathrm{NMR}\left(100 \mathrm{MHz}, \mathrm{CDCl}_{3}\right): \delta 160.8(\mathrm{~m}), 144.5$ (m), $139.8(\mathrm{~m}), 137.4(\mathrm{~m}), 129.2(\mathrm{t}, J=10 \mathrm{~Hz}), 123.6,118.9,113.7$ (t, $J=10 \mathrm{~Hz}), 112.2,111.4(\mathrm{~d}$, $J=25 \mathrm{~Hz}) ;{ }^{19} \mathrm{~F}$ NMR $\left(376 \mathrm{~Hz}, \mathrm{CDCl}_{3}\right)-112.76(2 \mathrm{~F}),-142.85(2 \mathrm{~F}),-155.88(1 \mathrm{~F}),-163.18(2 \mathrm{~F})$. IR $\left(v \mathrm{~cm}^{-1}\right) 2927,1899,1513$. HRMS $(\mathrm{EI})\left[\left(\mathrm{C}_{14} \mathrm{H}_{5} \mathrm{~F}_{7}\right)^{+}\right]$calcd $=306.0279$, found $\mathrm{m} / \mathrm{z}=306.0278$.

\section{(E)-1-(2,4-bis(trifluoromethyl)styryl)-2,3,4,5,6-pentafluorobenzene (21)}

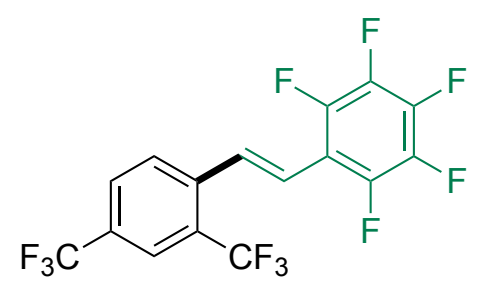

Thick oil, $88.3 \mathrm{mg}(87 \%), R_{\mathrm{f}} 0.48$ (1:19 EtOAc:hexanes). ${ }^{1} \mathrm{H}$ NMR (400 MHz, $\left.\mathrm{CDCl}_{3}\right): \delta 7.95$ (s, 1H), 7.91-7.84 (m, 3H), $7.03(\mathrm{~d}, J=16 \mathrm{~Hz}, 1 \mathrm{H}) ;{ }^{13} \mathrm{C}$ NMR (100 MHz, $\left.\mathrm{CDCl}_{3}\right): \delta 145.1(\mathrm{dm}, J=$ $250 \mathrm{~Hz}), 140.7(\mathrm{dm}, J=256 \mathrm{~Hz}), 139.1,136.5,131.4,130.6$ (q, $J=33 \mathrm{~Hz}), 128.9,128.6,128.3$, $127.8,123.3$ (q, $J=272 \mathrm{~Hz}), 123.2$ (q, $J=271 \mathrm{~Hz}), 119.0,111.2 ;{ }^{19} \mathrm{~F}$ NMR $\left(376 \mathrm{~Hz}, \mathrm{CDCl}_{3}\right)$ $60.10(3 \mathrm{~F}),-63.05(3 \mathrm{~F}),-141.89(2 \mathrm{~F}),-153.82(1 \mathrm{~F}),-162.14(2 \mathrm{~F})$. IR $\left(\mathrm{v} \mathrm{cm}^{-1}\right) 1900(\mathrm{w}), 1710(\mathrm{w})$, $1561(\mathrm{~m}) . \operatorname{HRMS}(\mathrm{EI})\left[\left(\mathrm{C}_{16} \mathrm{H}_{5} \mathrm{~F}_{11}\right)^{+}\right]$calcd $=406.0216$, found $\mathrm{m} / \mathrm{z}=406.0217$. 
(E)-1-methoxy-2-styrylbenzene (22) $)^{14}$

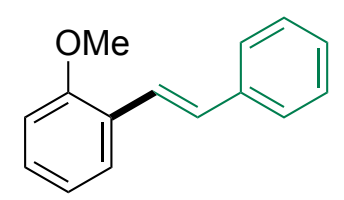

White solid, $47.7 \mathrm{mg}(91 \%), R_{\mathrm{f}} 0.4$ (hexanes). ${ }^{1} \mathrm{H}$ NMR (400 MHz, $\left.\mathrm{CDCl}_{3}\right): \delta 7.61$ (d, J=8 Hz, 1H), 7.56-7.48 (m, 3H), 7.37-7.34 (m, 2H), 7.26-7.23 (m, 2H), 7.12 (d, J=16 Hz, 1H), 7.00-6.90 (m, 2H), $3.90(\mathrm{~s}, 3 \mathrm{H})$.

\section{(E)-1,2,3,4,5-pentafluoro-6-(2-methoxystyryl)benzene (23)}

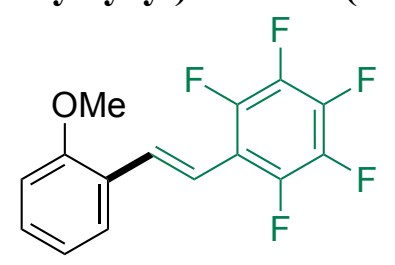

Off-white solid, $\mathrm{mp}=147-148{ }^{\circ} \mathrm{C}, 63 \mathrm{mg}(84 \%), R_{\mathrm{f}} 0.30$ (hexanes). ${ }^{1} \mathrm{H}$ NMR $\left(400 \mathrm{MHz}, \mathrm{CDCl}_{3}\right)$ : $\delta 7.76(\mathrm{~d}, J=16 \mathrm{~Hz}, 1 \mathrm{H}), 7.57(\mathrm{~d}, J=8 \mathrm{~Hz}, 1 \mathrm{H}), 7.33-7.30(\mathrm{~m}, 1 \mathrm{H}), 7.02-6.98(\mathrm{~m}, 2 \mathrm{H}), 6.91(\mathrm{~d}, J$ $=8 \mathrm{~Hz}, 1 \mathrm{H}), 3.90(\mathrm{~s}, 3 \mathrm{H}) ;{ }^{13} \mathrm{C} \mathrm{NMR}\left(100 \mathrm{MHz}, \mathrm{CDCl}_{3}\right): \delta 157.6,144.7$ (d, J=250 Hz), 140.6, 1, $137.6(\mathrm{~d}, J=249 \mathrm{~Hz}), 132.4,130,126.9,125.5,120.7,113.1,110.9,55.5 ;{ }^{19} \mathrm{~F}$ NMR $(376 \mathrm{MHz}$, $\left.\mathrm{CDCl}_{3}\right)$ : -142.98.12 (2F), -157.27 (1F), -163.34 (2F). IR ( $\left.\mathrm{v} \mathrm{cm}^{-1}\right) 2921(\mathrm{w}), 1916(\mathrm{w}), 1411(\mathrm{~s})$, 1321(s). HRMS (EI) $\left[\left(\mathrm{C}_{15} \mathrm{H}_{9} \mathrm{~F}_{5} \mathrm{O}\right)^{+}\right]$calcd $=300.0574$, found $\mathrm{m} / \mathrm{z}=300.0571$.

\section{(E)-2-(2-(perfluorophenyl)vinyl)benzofuran (24)}

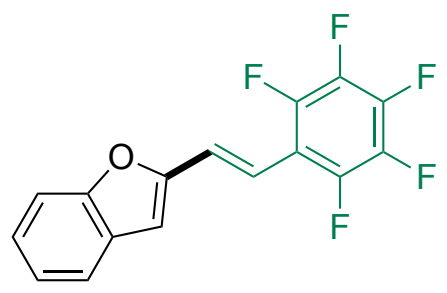

Sticky solid, $50.4 \mathrm{mg}(65 \%), R_{\mathrm{f}} 0.31$ (1:9 EtOAc:hexanes). ${ }^{1} \mathrm{H}$ NMR (400 MHz, $\left.\mathrm{CDCl}_{3}\right): \delta 7.57$ $(\mathrm{d}, J=8 \mathrm{~Hz}, 1 \mathrm{H}), 7.50(\mathrm{~d}, J=8 \mathrm{~Hz}, 1 \mathrm{H}), 7.36-7.31(\mathrm{~m}, 2 \mathrm{H}), 7.25-7.19(\mathrm{~m}, 2 \mathrm{H}), 6.81(\mathrm{~s}, 1 \mathrm{H}) ;{ }^{13} \mathrm{C}$ NMR (100 MHz, $\left.\mathrm{CDCl}_{3}\right): \delta 155.2,153.7,144.9(\mathrm{~d}, J=251 \mathrm{~Hz}), 140.7$ (d, $\left.J=256 \mathrm{~Hz}\right), 138.9$, 136.5, 128.7, 125.6, 124.14, 123.2, 121.3, 113.8, 111.2, 108.1; ${ }^{19} \mathrm{~F}$ NMR (376 Hz, $\left.\mathrm{CDCl}_{3}\right)-142.26$ 
(2F), -155.65 (1F), -162.73 (2F). IR ( $\left.v \mathrm{~cm}^{-1}\right) 2917(\mathrm{~m}), 1926(\mathrm{w}), 1631(\mathrm{~s}), 1424(\mathrm{~m})$. HRMS (EI) $\left[\left(\mathrm{C}_{16} \mathrm{H}_{7} \mathrm{~F}_{5} \mathrm{O}\right)^{+}\right]$calcd $=310.0417$, found $\mathrm{m} / \mathrm{z}=310.0410$.

(E)-2,4-dimethyl-1-styrylbenzene $(25)^{15}$

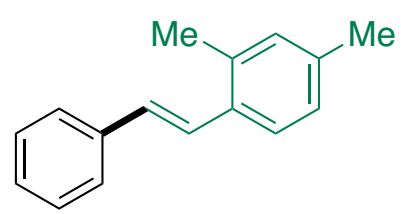

Waxy solid, $37.9 \mathrm{mg}$ (73\%), $R_{\mathrm{f}} 0.41$ (1:19, EtOAc:hexanes). ${ }^{1} \mathrm{H}$ NMR (400 MHz, $\left.\mathrm{CDCl}_{3}\right): \delta 7.59$ $\left(\mathrm{dd}, J_{1}=4 \mathrm{~Hz}, J_{2}=8 \mathrm{~Hz}, 3 \mathrm{H}\right), 7.37-7.32(\mathrm{~m}, 2 \mathrm{H}), 7.28-7.24(\mathrm{~m}, 2 \mathrm{H}), 7.03-7(\mathrm{~m}, 2 \mathrm{H}), 6.95(\mathrm{~d}, J=$ $20 \mathrm{~Hz}, 1 \mathrm{H}), 2.39(\mathrm{~s}, 3 \mathrm{H}), 2.32(\mathrm{~s}, 3 \mathrm{H})$.

(E)-1-methoxy-4-(4-(trifluoromethyl)styryl)benzene $(26)^{16}$<smiles>COc1ccc(/C=C/c2ccc(C(F)(F)F)cc2)cc1</smiles>

Off-white solid, $56.3 \mathrm{mg}(81 \%), R_{\mathrm{f}} 0.33$ (1:19, EtOAc:hexanes). ${ }^{1} \mathrm{H}$ NMR (400 MHz, $\left.\mathrm{CDCl}_{3}\right): \delta$ 7.6-7.55 (m, 4H), $7.48(\mathrm{~d}, J=8 \mathrm{~Hz}, 2 \mathrm{H}), 7.15(\mathrm{~d}, J=16 \mathrm{~Hz}, 1 \mathrm{H}), 6.98(\mathrm{~d}, J=16 \mathrm{~Hz}, 1 \mathrm{H}), 6.92(\mathrm{~d}$, $J=8 \mathrm{~Hz}, 2 \mathrm{H}), 3.84(\mathrm{~s}, 3 \mathrm{H}) ;{ }^{19} \mathrm{~F}$ NMR $\left(376 \mathrm{~Hz}, \mathrm{CDCl}_{3}\right)-62.39(3 \mathrm{~F})$.

(E)-1-styrylnaphthalene (27) ${ }^{17}$

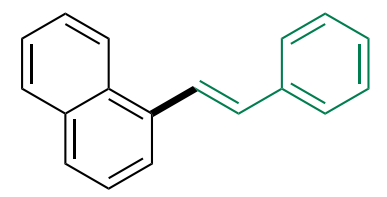

White solid, $38 \mathrm{mg}(66 \%), R_{\mathrm{f}} 0.6$ ('Hexanes). ${ }^{1} \mathrm{H} \mathrm{NMR}\left(500 \mathrm{MHz}, \mathrm{CDCl}_{3}\right): \delta 8.23$ (d, J=10 Hz, $1 \mathrm{H}), 7.96-7.75(\mathrm{~m}, 4 \mathrm{H}), 7.62-7.27(\mathrm{~m}, 8 \mathrm{H}), 7.16(\mathrm{~d}, J=16 \mathrm{~Hz}, 1 \mathrm{H}), 6.91(\mathrm{~d}, J=20 \mathrm{~Hz}, 1 \mathrm{H})$. 


\section{(E)-1-methyl-4-(4-(trifluoromethyl)styryl)benzene (28) $)^{18}$}

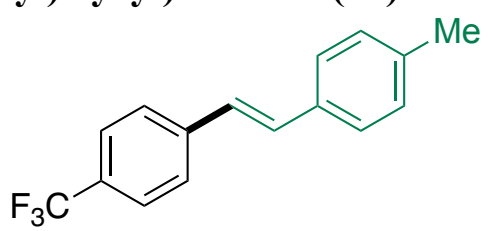

Colorless liquid, $55.6 \mathrm{mg}$ (85\%), $R_{\mathrm{f}} 0.37$ (1:19, EtOAc:hexanes). ${ }^{1} \mathrm{H}$ NMR (400 MHz, $\left.\mathrm{CDCl}_{3}\right): \delta$ 7.62-7.57 (m, 4H), 7.44 (d, $J=8 \mathrm{~Hz}, 2 \mathrm{H}), 7.21-7.05$ (m, 4H) 2.38 (s, 3H); ${ }^{19} \mathrm{~F}$ NMR (376 Hz, $\left.\mathrm{CDCl}_{3}\right)-62.42(3 \mathrm{~F})$.

Methyl (E)-3-(4-(trifluoromethyl)phenyl)acrylate (29) ${ }^{11}$

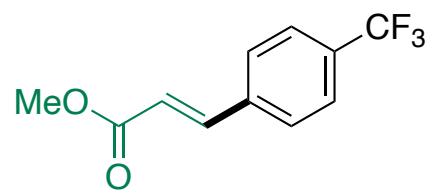

Off-white solid, $46 \mathrm{mg}$ (80\%), $R_{\mathrm{f}} 0.35$ (hexanes). ${ }^{1} \mathrm{H}$ NMR (400 MHz, $\left.\mathrm{CDCl}_{3}\right): \delta 7.70$ (d, $J=16$ $\mathrm{Hz}, 1 \mathrm{H}), 7.66-7.61(\mathrm{~m}, 4 \mathrm{H}), 6.51(\mathrm{~d}, J=16 \mathrm{~Hz}, 1 \mathrm{H}), 3.83(\mathrm{~s}, 3 \mathrm{H}) ;{ }^{19} \mathrm{~F}$ NMR $\left(376 \mathrm{~Hz}, \mathrm{CDCl}_{3}\right)-$ $62.82(3 \mathrm{~F})$.

Methyl (E)-3-(2,6-difluorophenyl)acrylate (30) ${ }^{19}$<smiles>COC(=O)/C=C/c1c(F)cccc1F</smiles>

Colorless liquid, $35 \mathrm{mg}(70 \%), R_{\mathrm{f}} 0.33$ (hexanes). ${ }^{1} \mathrm{H} \mathrm{NMR}\left(400 \mathrm{MHz}, \mathrm{CDCl}_{3}\right): \delta 7.78(\mathrm{~d}, J=16$ $\mathrm{Hz}, 1 \mathrm{H}), 7.30(\mathrm{t}, J=8 \mathrm{~Hz}, 1 \mathrm{H}), 6.92-6.96(\mathrm{~m}, 2 \mathrm{H}), 6.75(\mathrm{~d}, J=16 \mathrm{~Hz}, 1 \mathrm{H}), 3.82(\mathrm{~s}, 3 \mathrm{H}) ;{ }^{19} \mathrm{~F} \mathrm{NMR}$ $\left(376 \mathrm{~Hz}, \mathrm{CDCl}_{3}\right)-110.23(2 \mathrm{~F})$.

Methyl (E)-3-(2-nitrophenyl)acrylate $(31)^{20}$<smiles>COC(=O)/C=C/c1ccccc1[N+](=O)[O-]</smiles>

Yellow solid, $39.3 \mathrm{mg}(76 \%), R_{\mathrm{f}} 0.33$ (1:9, EtOAc:hexanes). ${ }^{1} \mathrm{H}$ NMR (400 MHz, $\left.\mathrm{CDCl}_{3}\right): \delta 8.12$ $(\mathrm{d}, J=16 \mathrm{~Hz}, 1 \mathrm{H}), 8.05(\mathrm{~d}, J=8 \mathrm{~Hz}, 1 \mathrm{H}), 7.64(\mathrm{t}, J=8 \mathrm{~Hz}, 2 \mathrm{H}), 7.55(\mathrm{~d}, J=8 \mathrm{~Hz}, 1 \mathrm{H}), 6.37(\mathrm{~d}$, $J=16 \mathrm{~Hz}, 1 \mathrm{H}), 3.83(\mathrm{~s}, 3 \mathrm{H})$. 
tert-butyl (E)-3-(4-(trifluoromethyl)phenyl)acrylate $(32)^{21}$

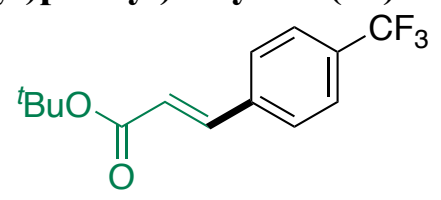

Colorless liquid, $57.1 \mathrm{mg}(84 \%), R_{\mathrm{f}} 0.41$ (1:9, EtOAc:hexanes). ${ }^{1} \mathrm{H}$ NMR (400 MHz, $\left.\mathrm{CDCl}_{3}\right): \delta$ 7.61-7.57 (m, 5H), $6.44(\mathrm{~d}, J=16 \mathrm{~Hz}, 1 \mathrm{H}), 7.64(\mathrm{t}, J=8 \mathrm{~Hz}, 2 \mathrm{H}), 1.54(\mathrm{~s}, 9 \mathrm{H})) ;{ }^{19} \mathrm{~F}$ NMR $(376$ $\left.\mathrm{Hz}, \mathrm{CDCl}_{3}\right)-62.87(3 \mathrm{~F})$. 


\section{REFERENCE}

(1) J. Brals, J. D. Smith, F. Ibrahim, F. Gallou, S. Handa, ACS Catal. 2017, 7, 7245-7250.

(2) G. R. Fulmer, A. J. M. Miller, N. H. Sherden, H. E. Gottlieb, A. Nudelman, B. M. Stoltz, J. E. Bercaw, K. I. Goldberg, Organometallics 2010, 29, 2176-2179.

(3) L. Chu, F. L. Qing, Org. Lett. 2010, 12, 5060-5063.

(4) D. VANDER JAGT, M. DECK, Lorraine, E. ROYER, Robert, Substituted stilbenes as inhibitors of $n f-k b$ and activators of NRf2, 2019, WO 2019/108773 Al.

(5) H.-J. Xu, Y.-Q. Zhao, X.-F. Zhou, J. Org. Chem. 2011, 76, 8036-8041.

(6) X. Zhang, S. Fan, C.-Y. He, X. Wan, Q.-Q. Min, J. Yang, Z.-X. Jiang, J. Am. Chem. Soc. 2010, $132,4506-4507$.

(7) L. M. Geary, P. G. Hultin, Org. Lett. 2009, 11, 5478-5481.

(8) L. Chen, Y. Hisaeda, H. Shimakoshi, Adv. Synth. Catal. 2019, 361, 2877-2884.

(9) C.-T. Cao, H. Yuan, Q. Zhu, C. Cao, J. Phys. Org. Chem. 2019, 32, e3962.

(10) D.-H. Lee, A. Taher, S. Hossain, M.-J. Jin, Org. Lett. 2011, 13, 5540-5543.

(11) Z. Xiong, N. Wang, M. Dai, A. Li, J. Chen, Z. Yang, Org. Lett. 2004, 6, 3337-3340.

(12) Z.-Y. Peng, F.-F. Ma, L.-F. Zhu, X.-M. Xie, Z. Zhang, J. Org. Chem. 2009, 74, 6855-6858.

(13) P. Du, J. L. Brosmer, D. G. Peters, Org. Lett. 2011, 13, 4072-4075.

(14) R. K. Arvela, N. E. Leadbeater, J. Org. Chem. 2005, 70, 1786-1790.

(15) T. T. Gao, A. P. Jin, L. X. Shao, Beilstein J. Org. Chem. 2012, 8, 1916-1919.

(16) G. Hilt, C. Hengst, J. Org. Chem. 2007, 72, 7337-7342.

(17) R. Cella, H. A. Stefani, Tetrahedron 2006, 62, 5656-5662.

(18) K. N. Sharma, N. Satrawala, A. K. Srivastava, M. Ali, R. K. Joshi, Org. Biomol. Chem. 2019, 17, 8969-8976.

(19) Z. Fu, S. Huang, W. Su, M. Hong, Org. Lett. 2010, 12, 4992-4995.

(20) Z. Zhang, Z. Zha, C. Gan, C. Pan, Y. Zhou, Z. Wang, M.-M. Zhou, J. Org. Chem. 2006, 71, 4339-4342.

(21) Zhu, M.-K.; Zhao, J.-F.; Loh, T.-P. Palladium-Catalyzed C-C Bond Formation of Arylhydrazines with Olefins via Carbon-Nitrogen Bond Cleavage. Org. Lett. 2011, 13, 6308-6311. 


\section{NMR SPECTRA}

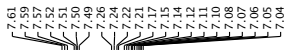<smiles>Fc1ccc(/C=C/c2ccccc2F)cc1</smiles>
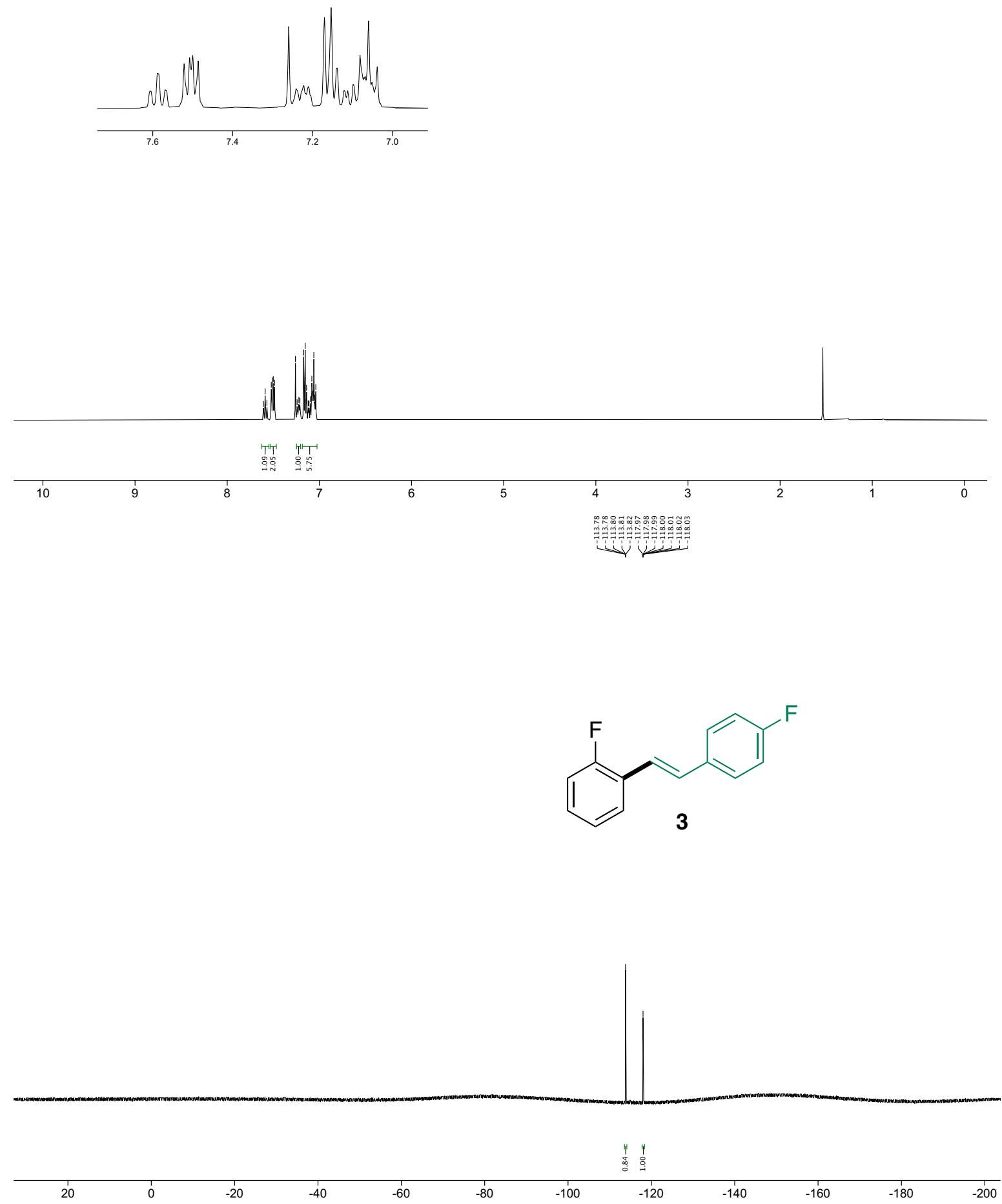


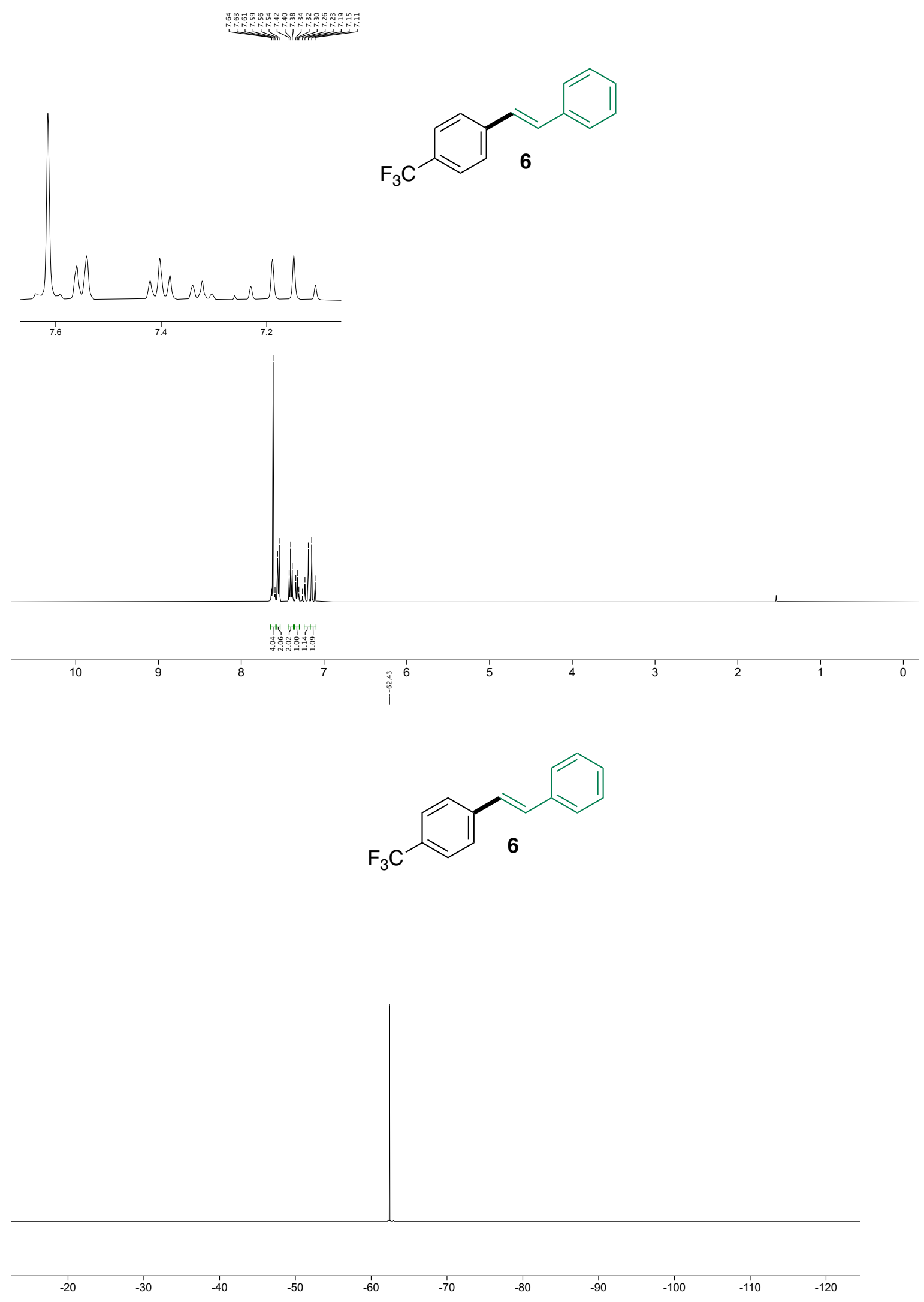




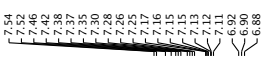
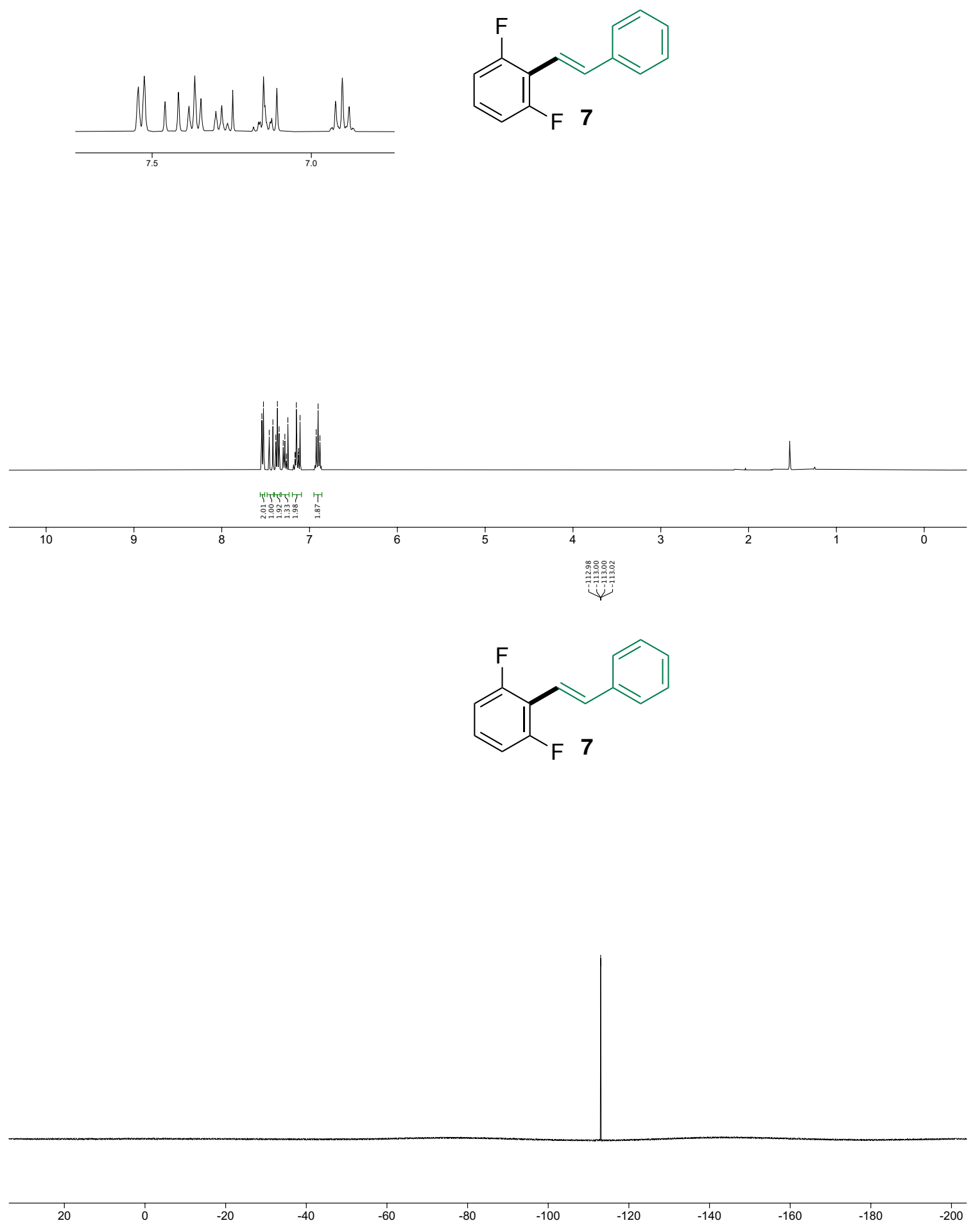

S37 

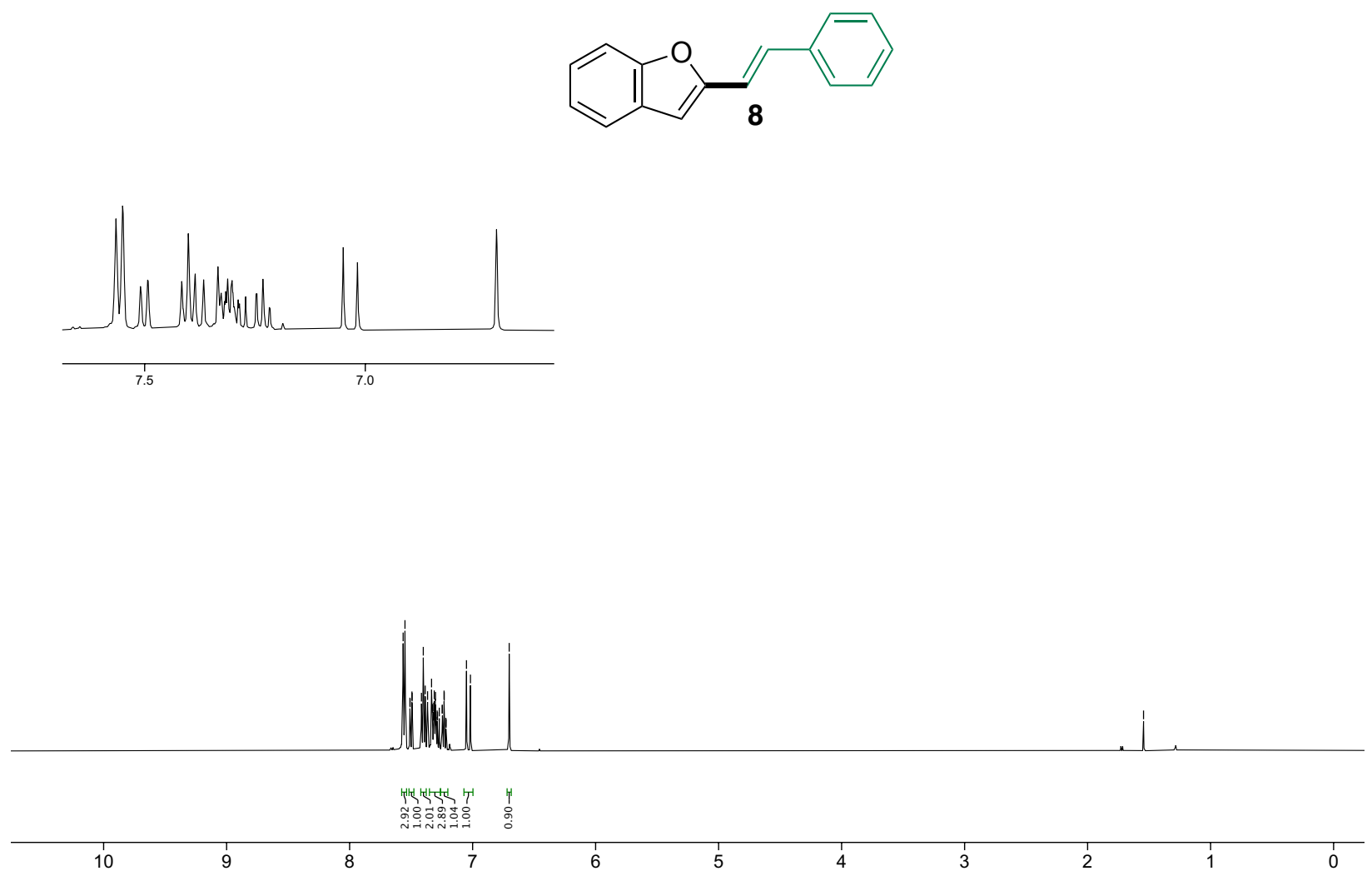

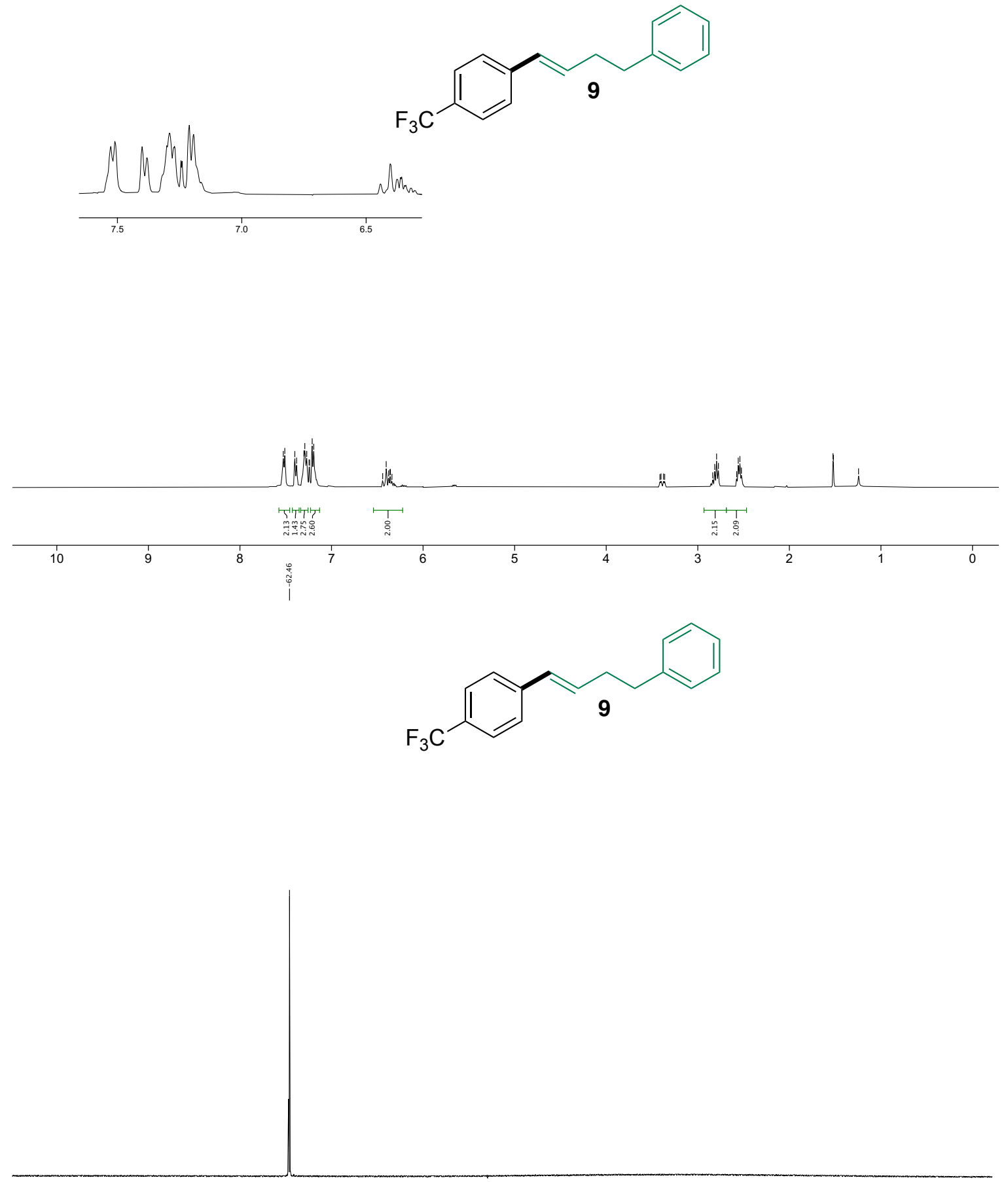

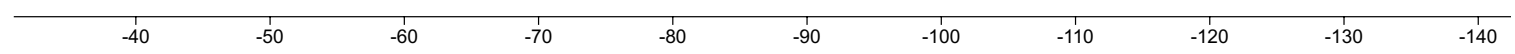



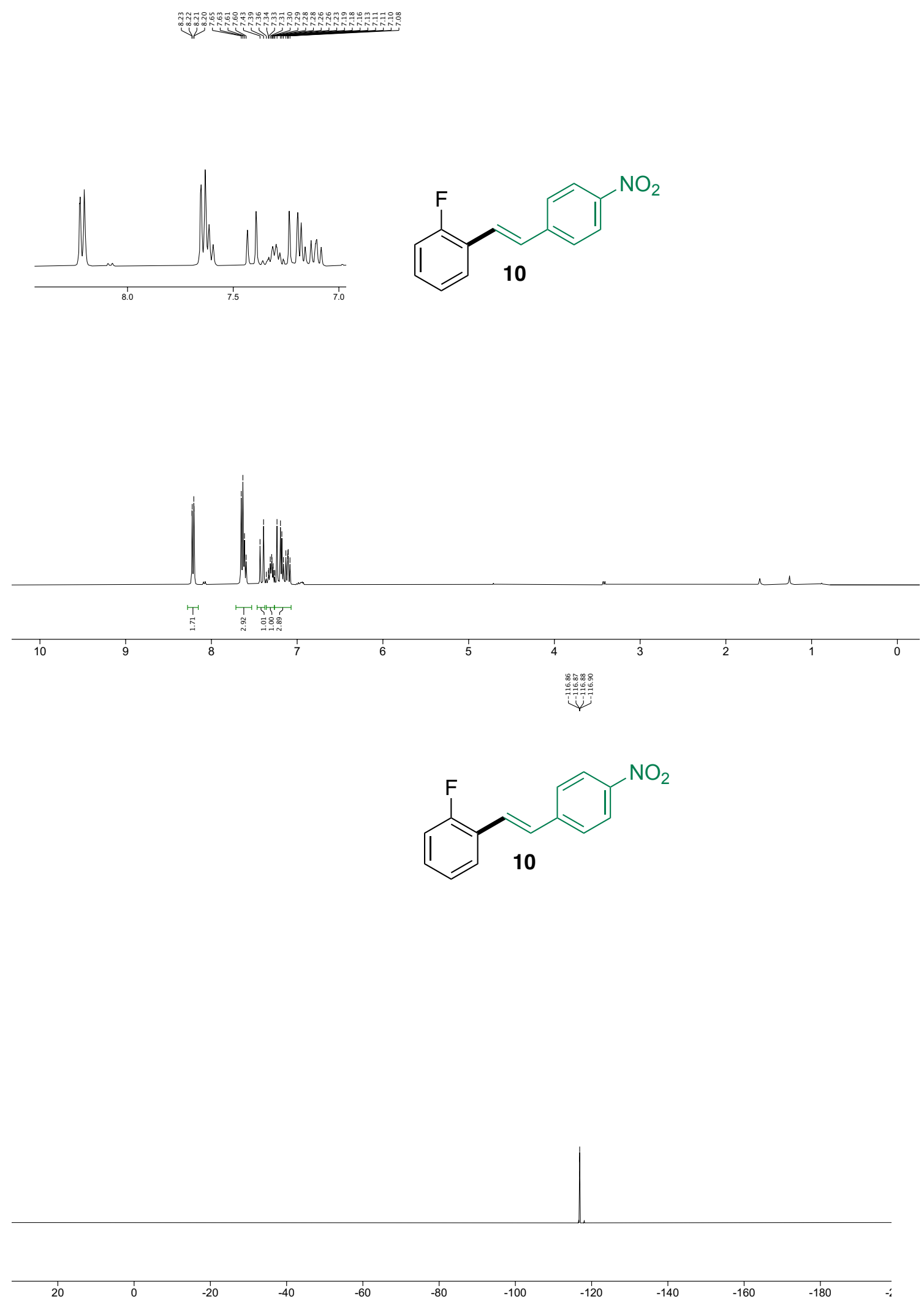


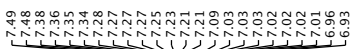

(II)
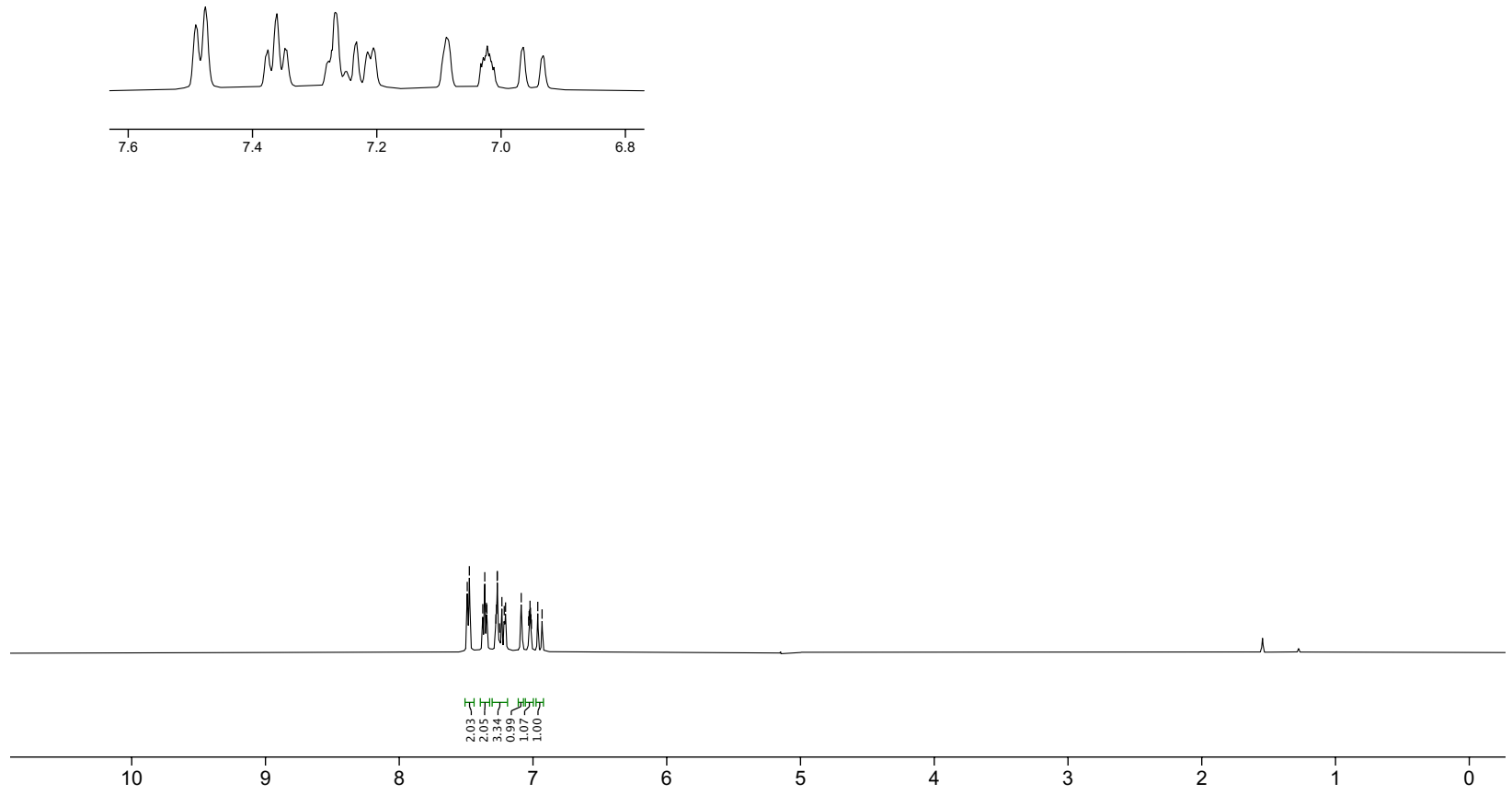

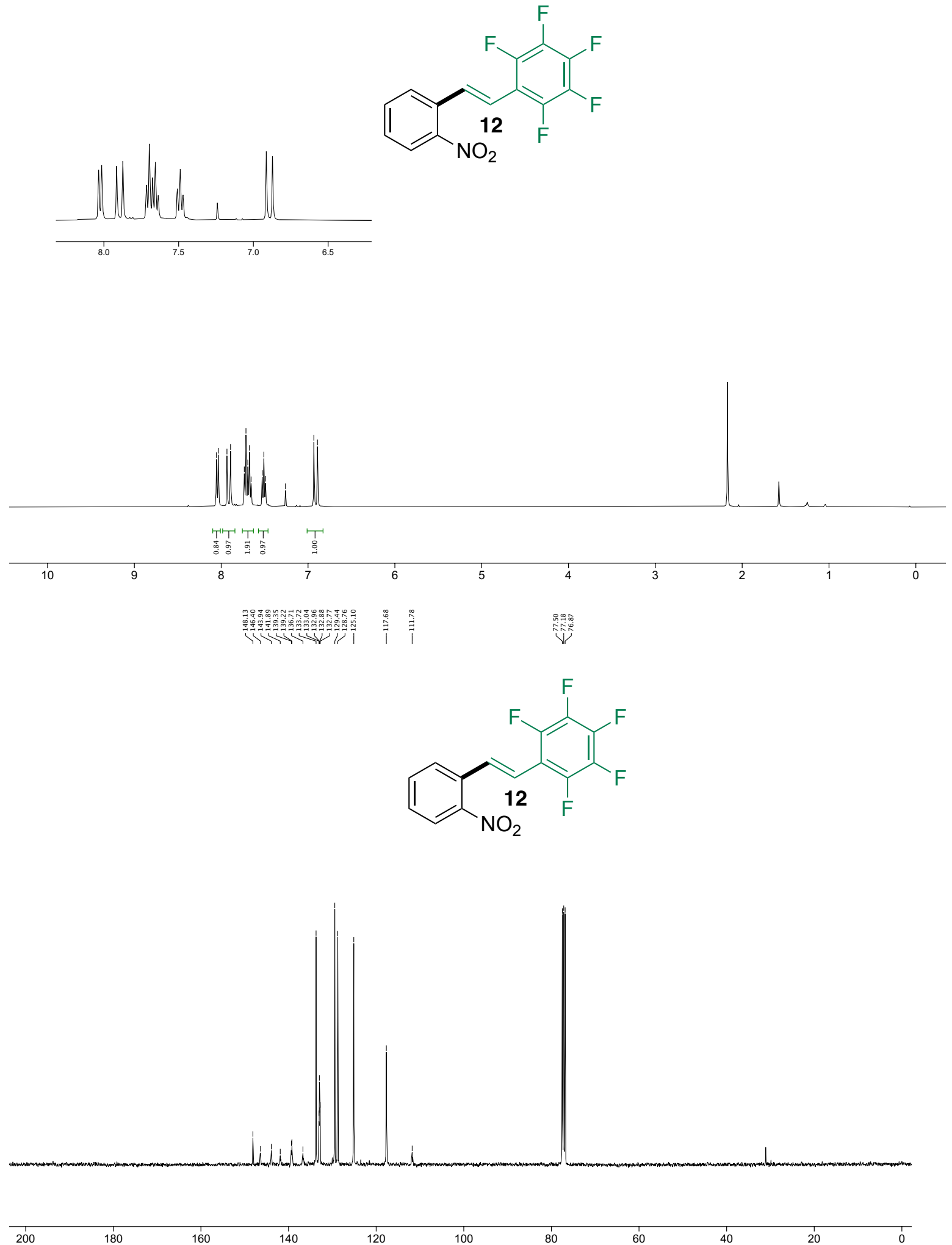


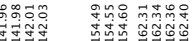

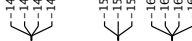
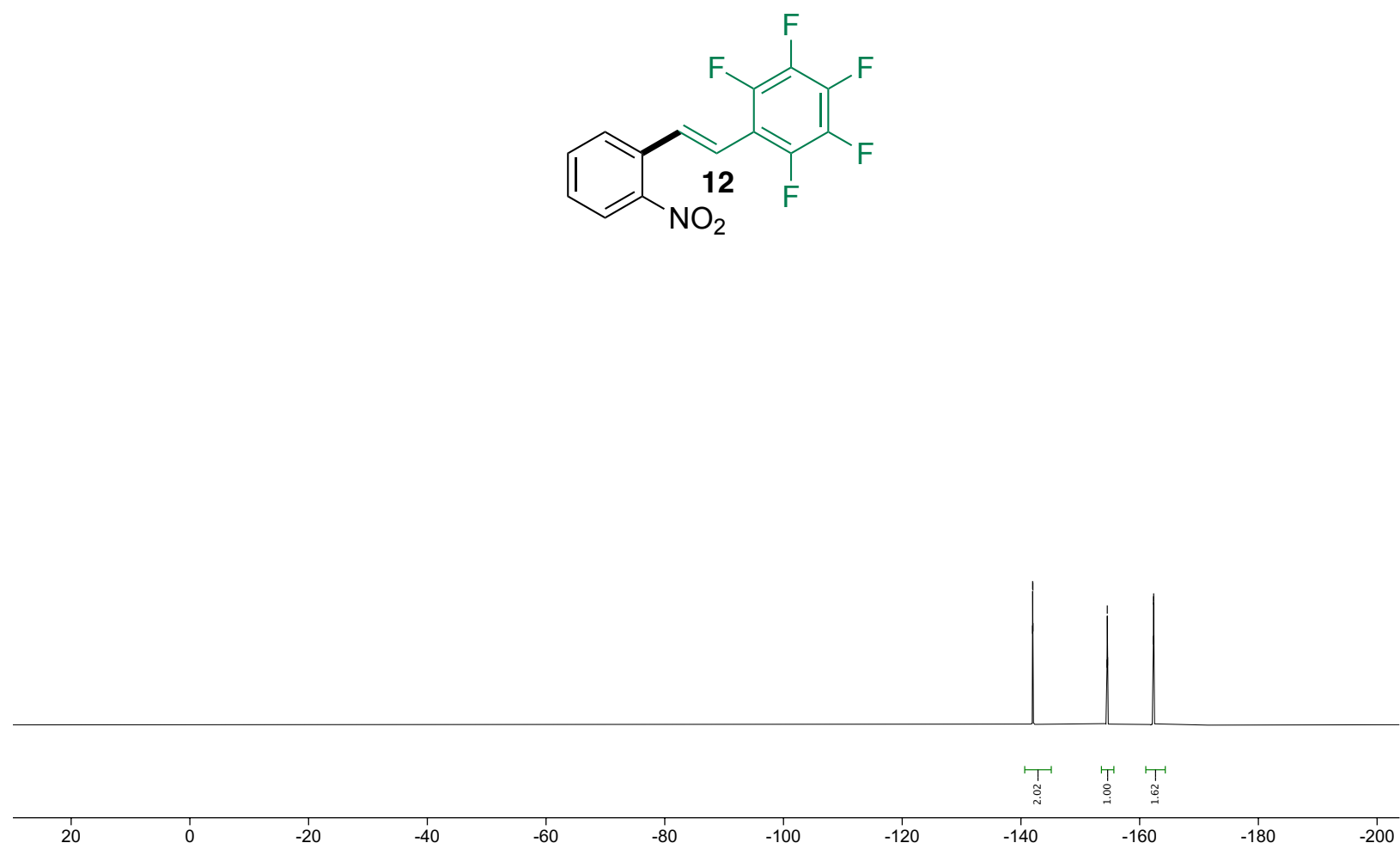

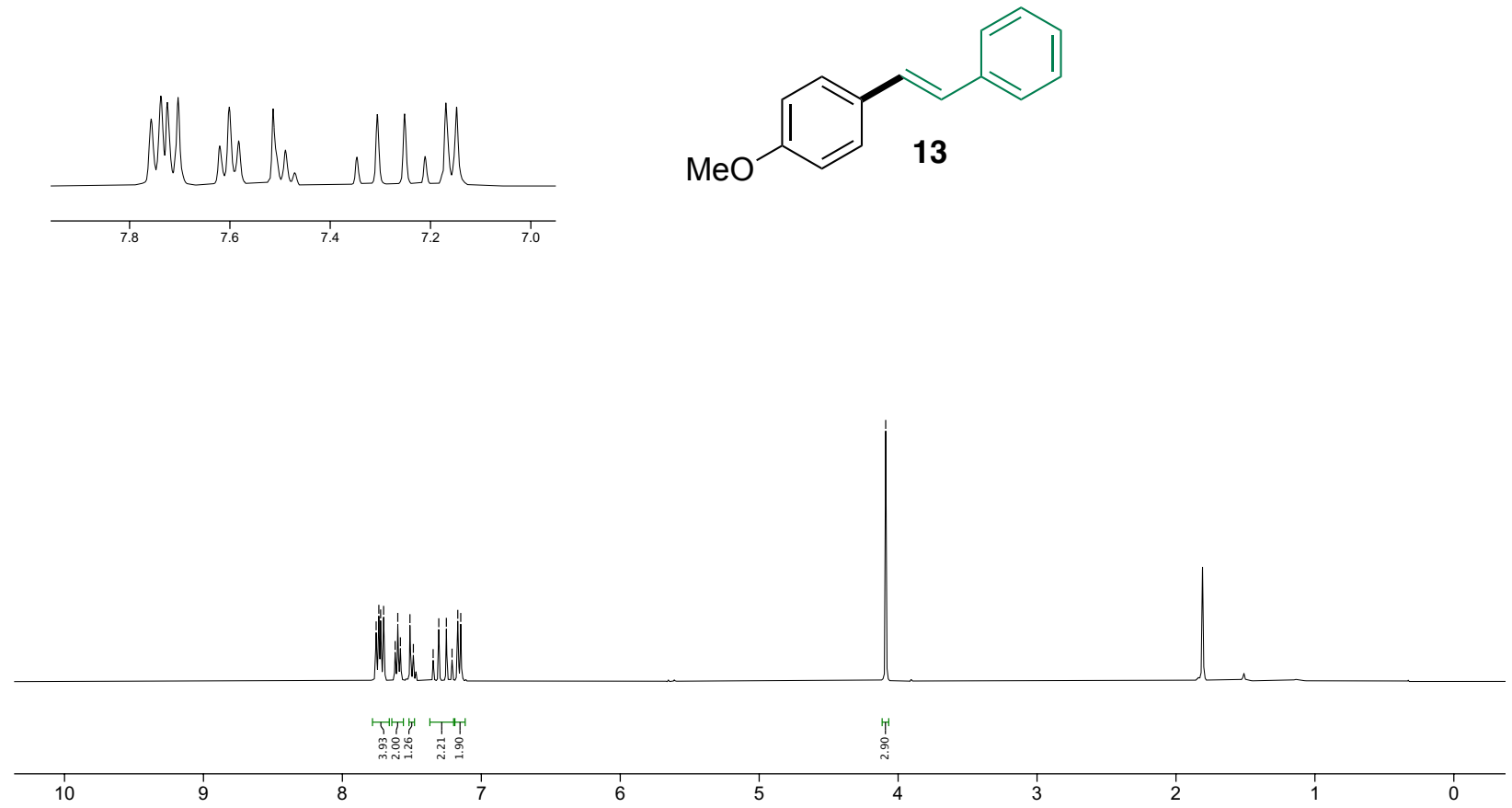

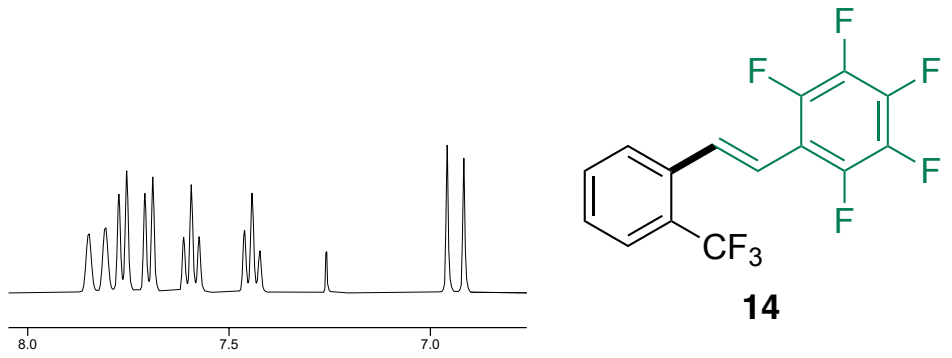

14

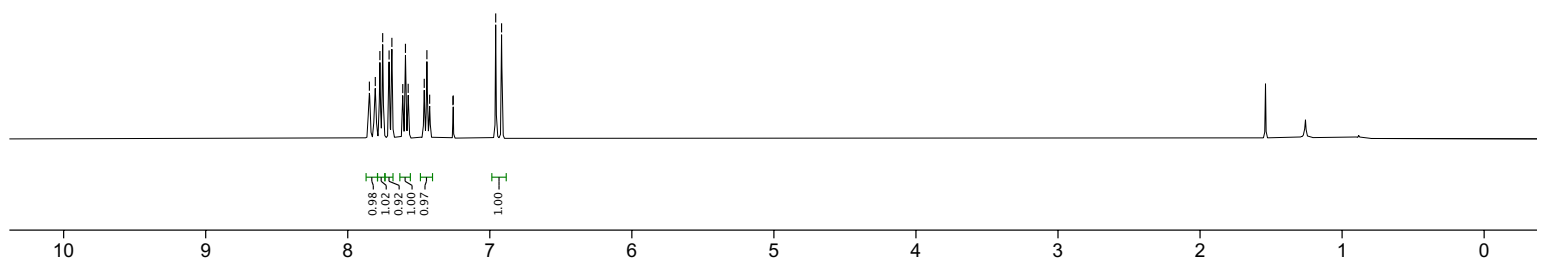

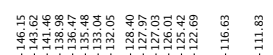
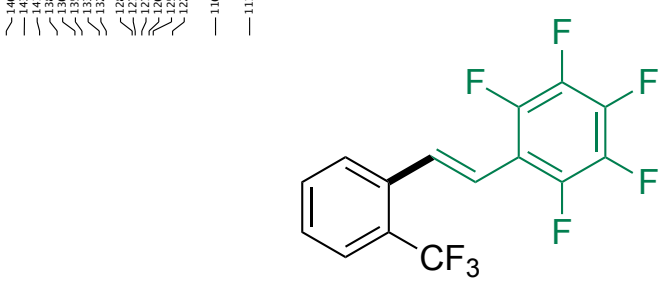

14

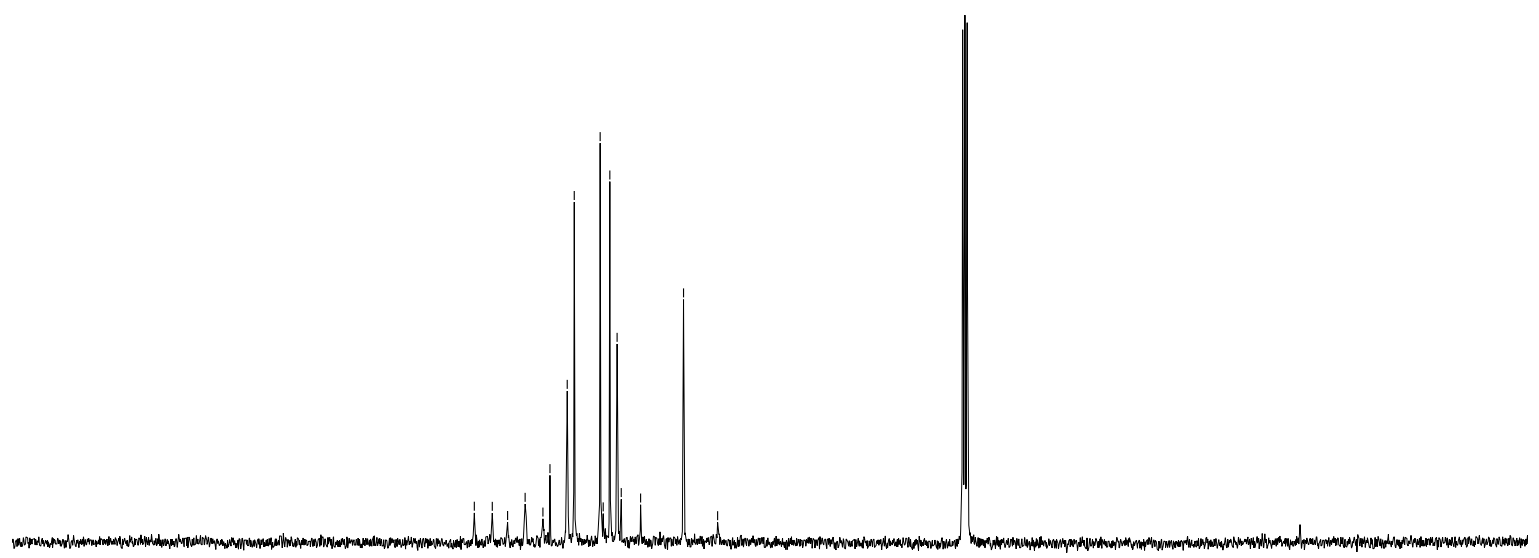

200

180

160

140

120

100

80

60

40

20 0 


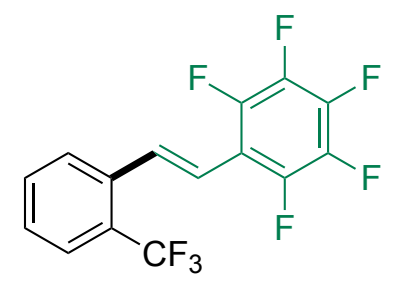

14

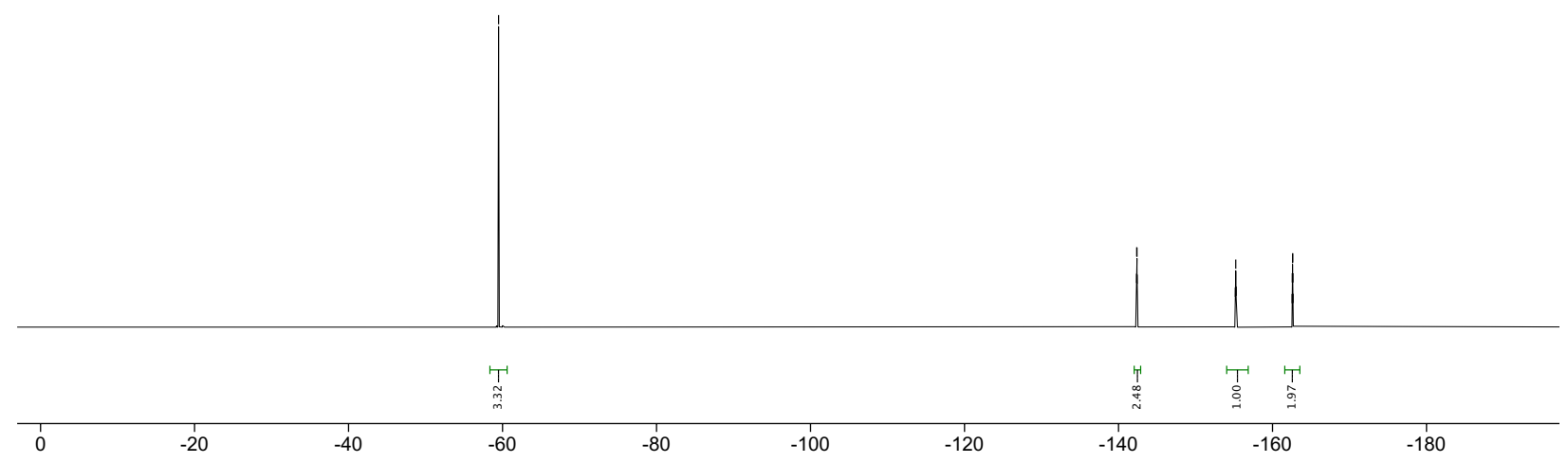



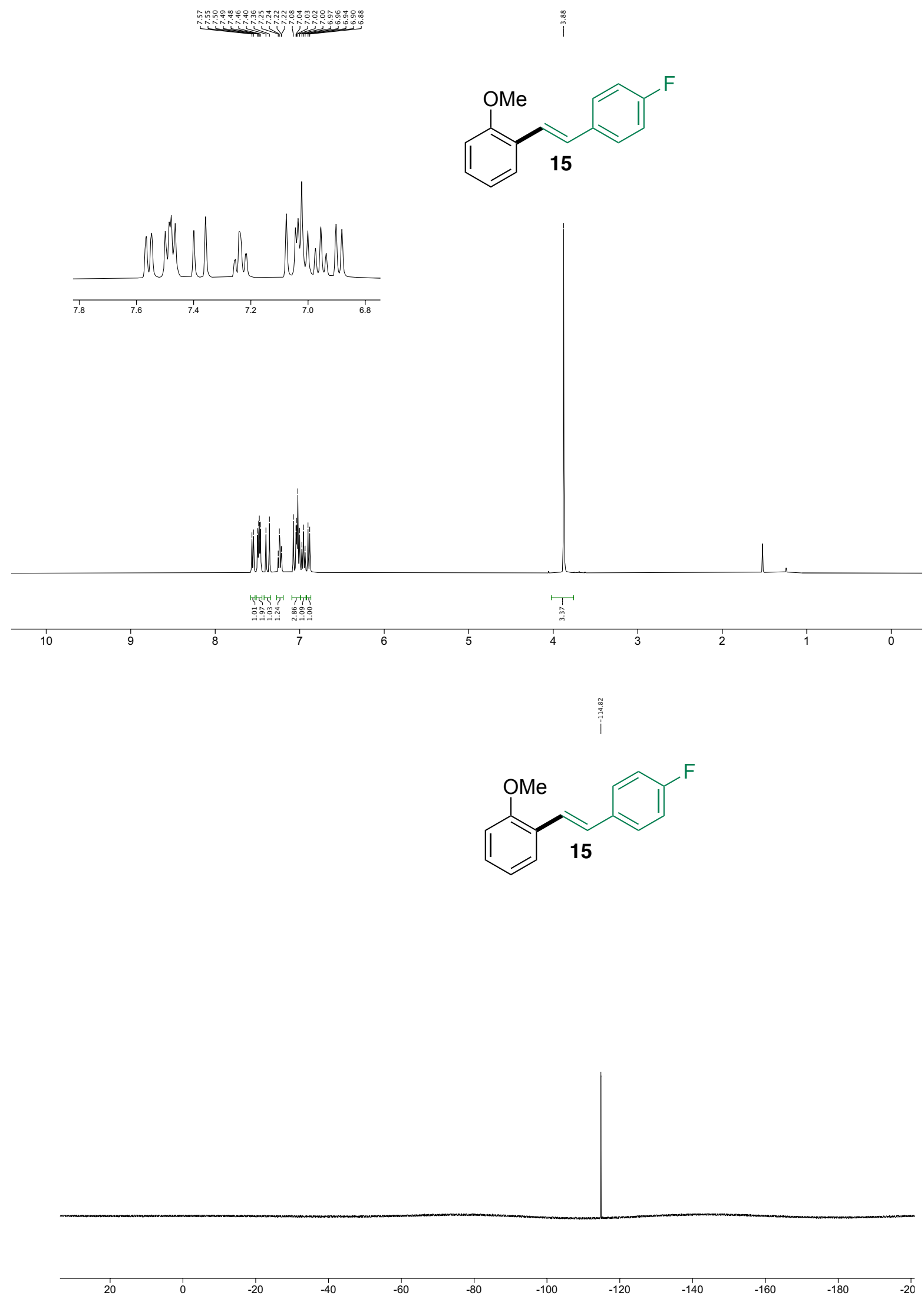

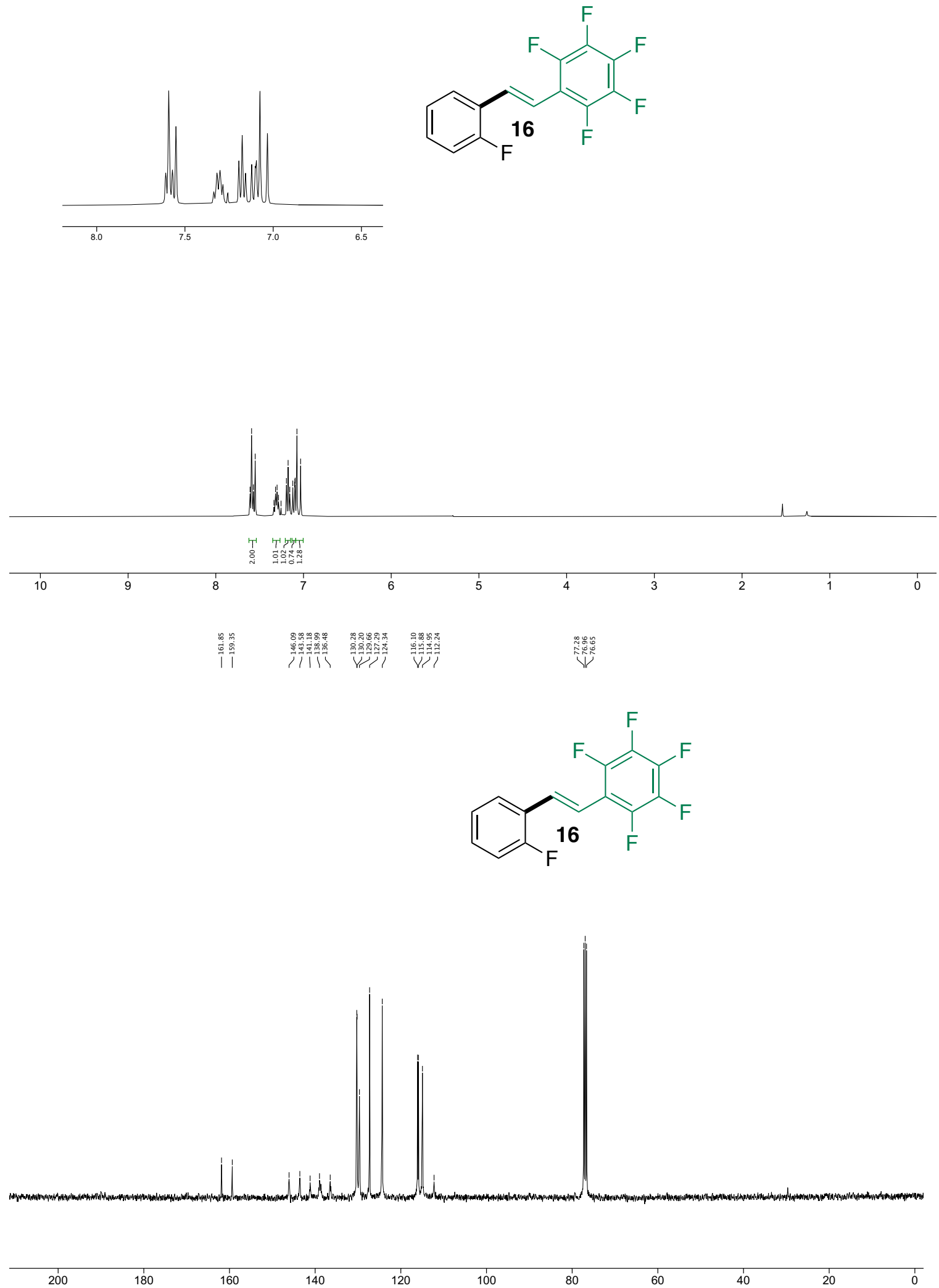

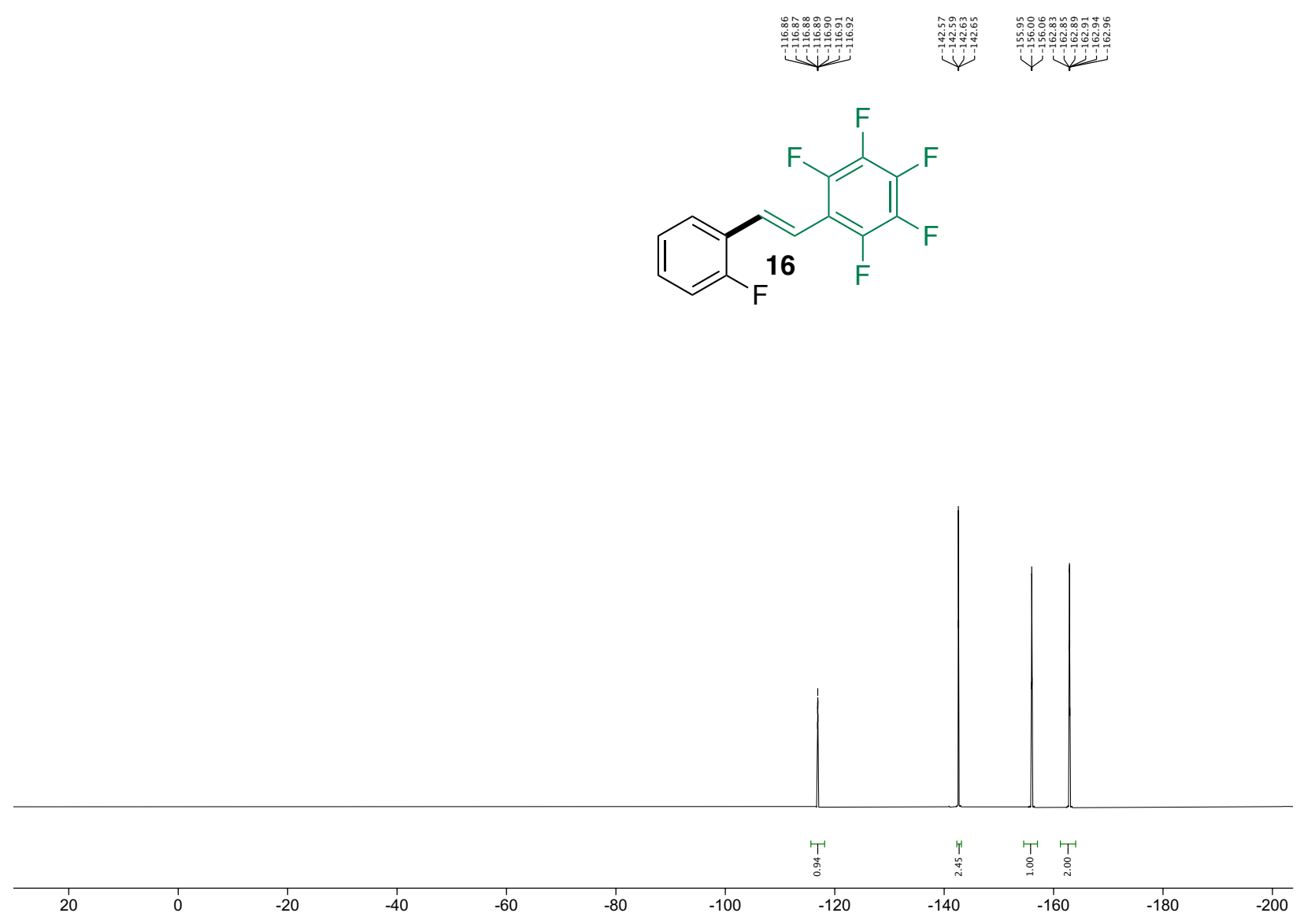


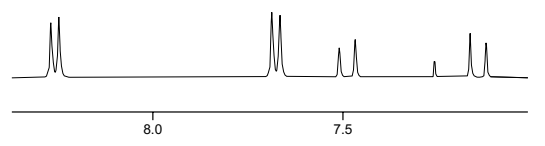<smiles>O=[N+]([O-])c1ccc(/C=C/c2c(F)c(F)c(F)c(F)c2F)cc1</smiles>
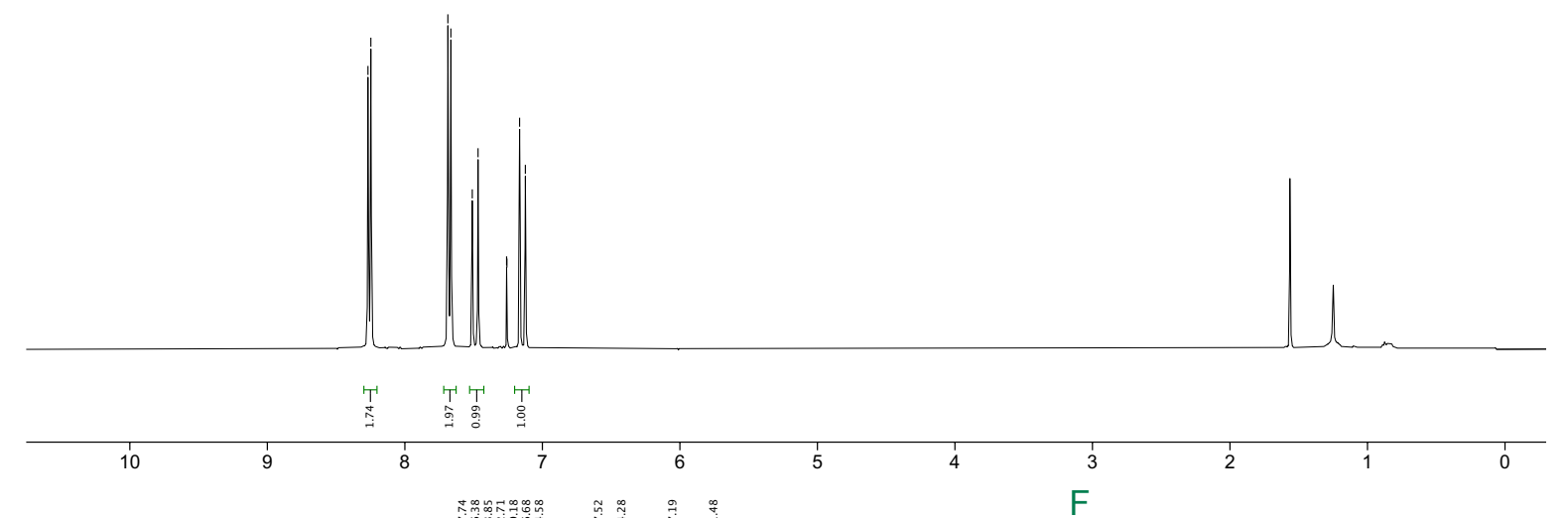

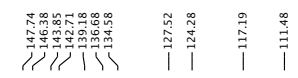

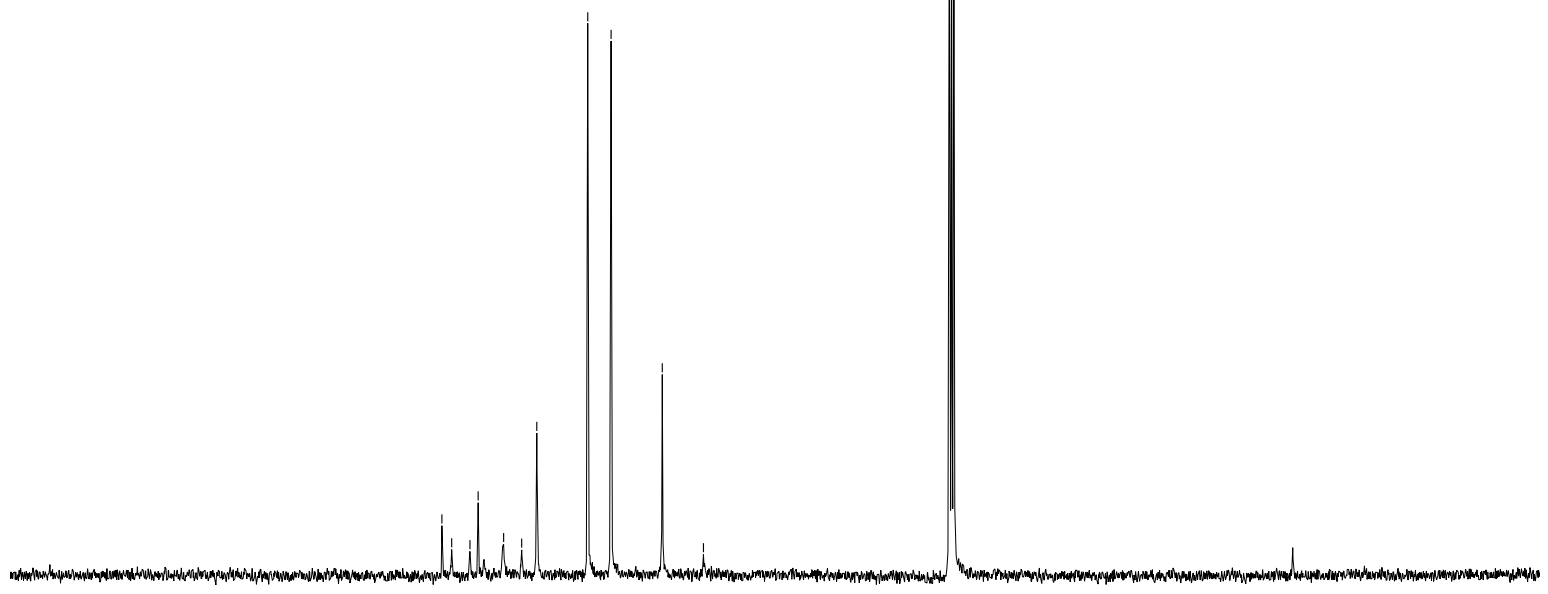



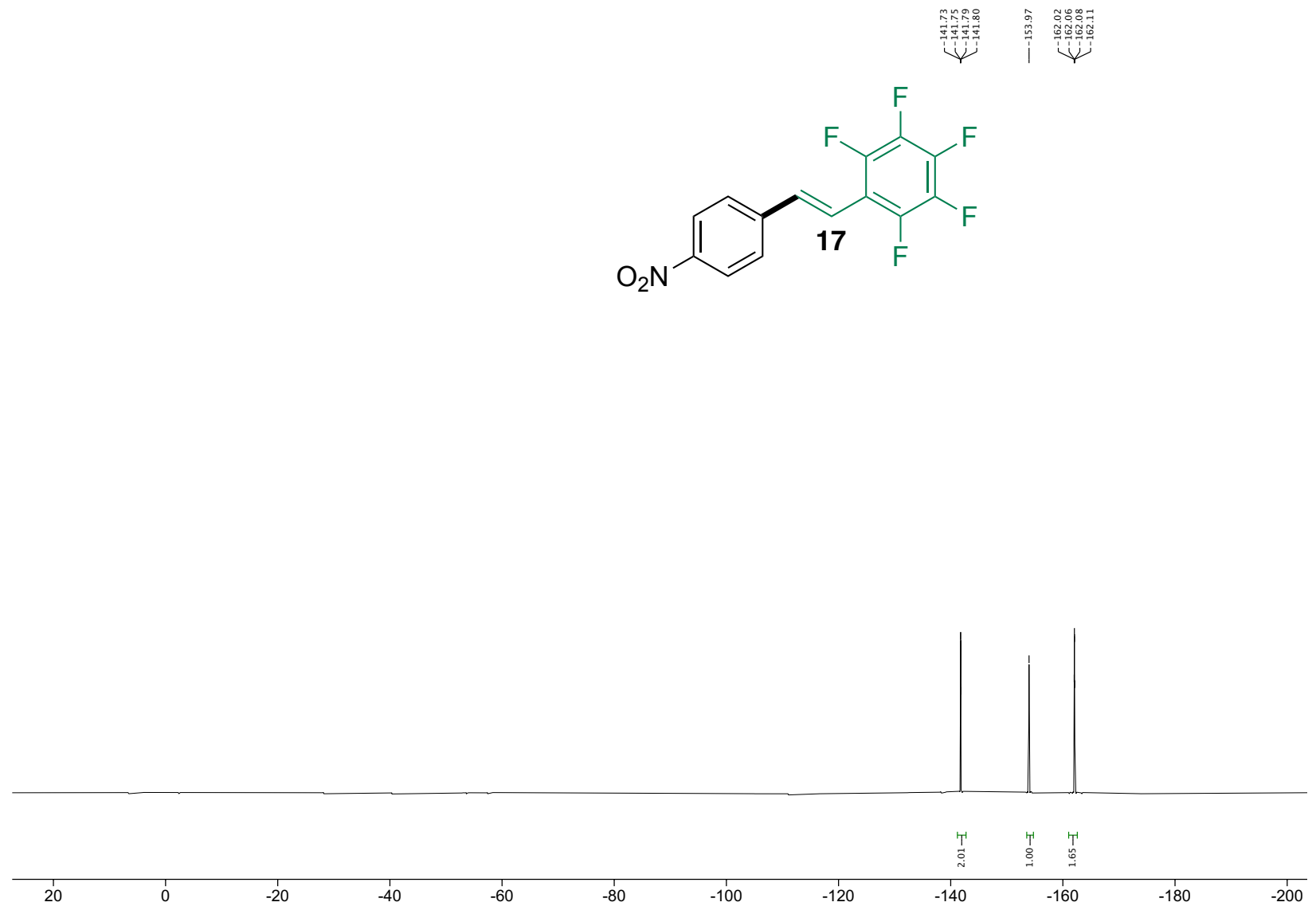


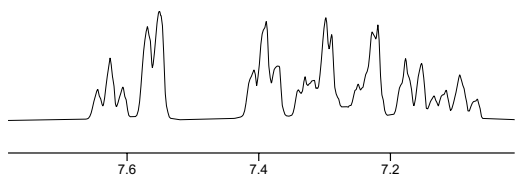<smiles>Fc1ccccc1/C=C/c1ccccc1</smiles>

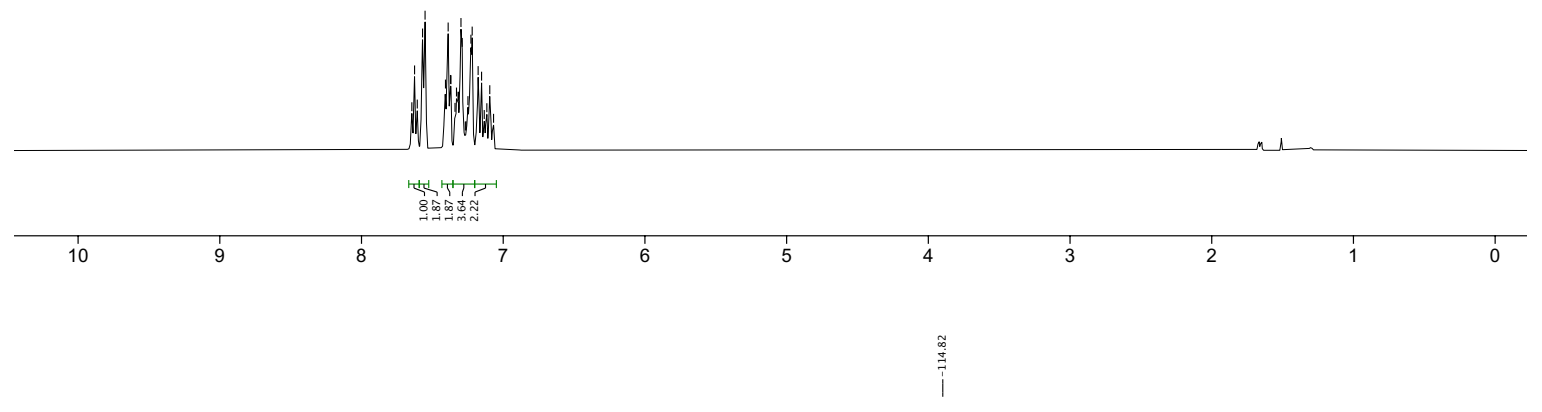<smiles>Fc1ccccc1/C=C/c1ccccc1</smiles>

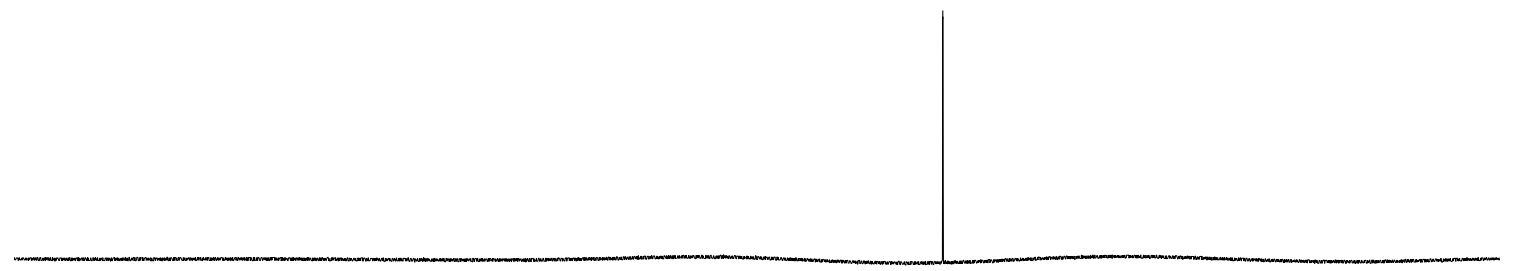

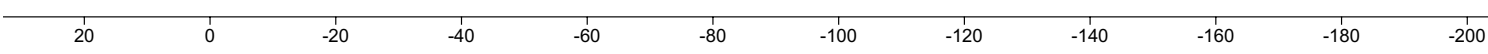




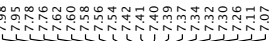

$\mathrm{NO}_{2}$

$\underbrace{2}_{19}$
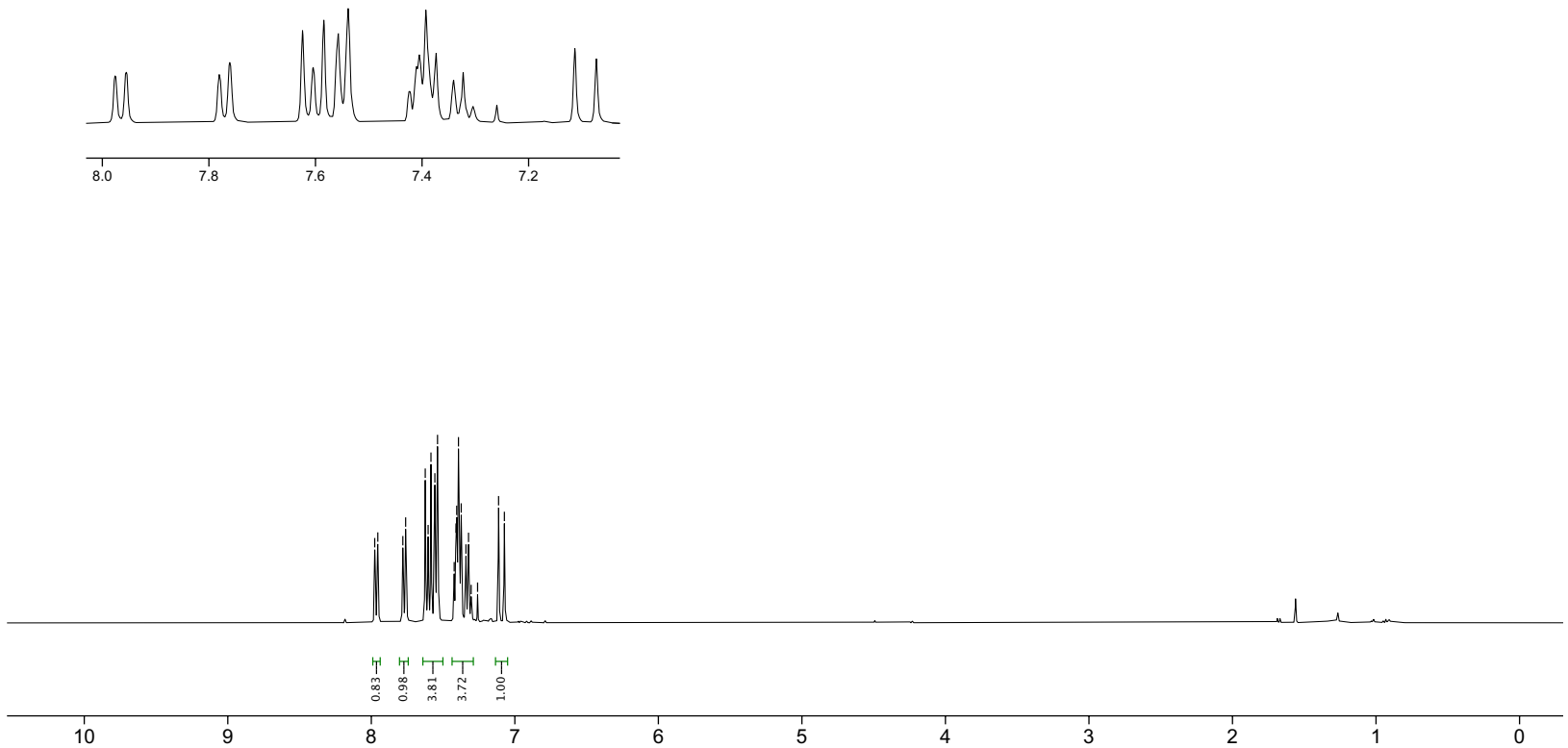

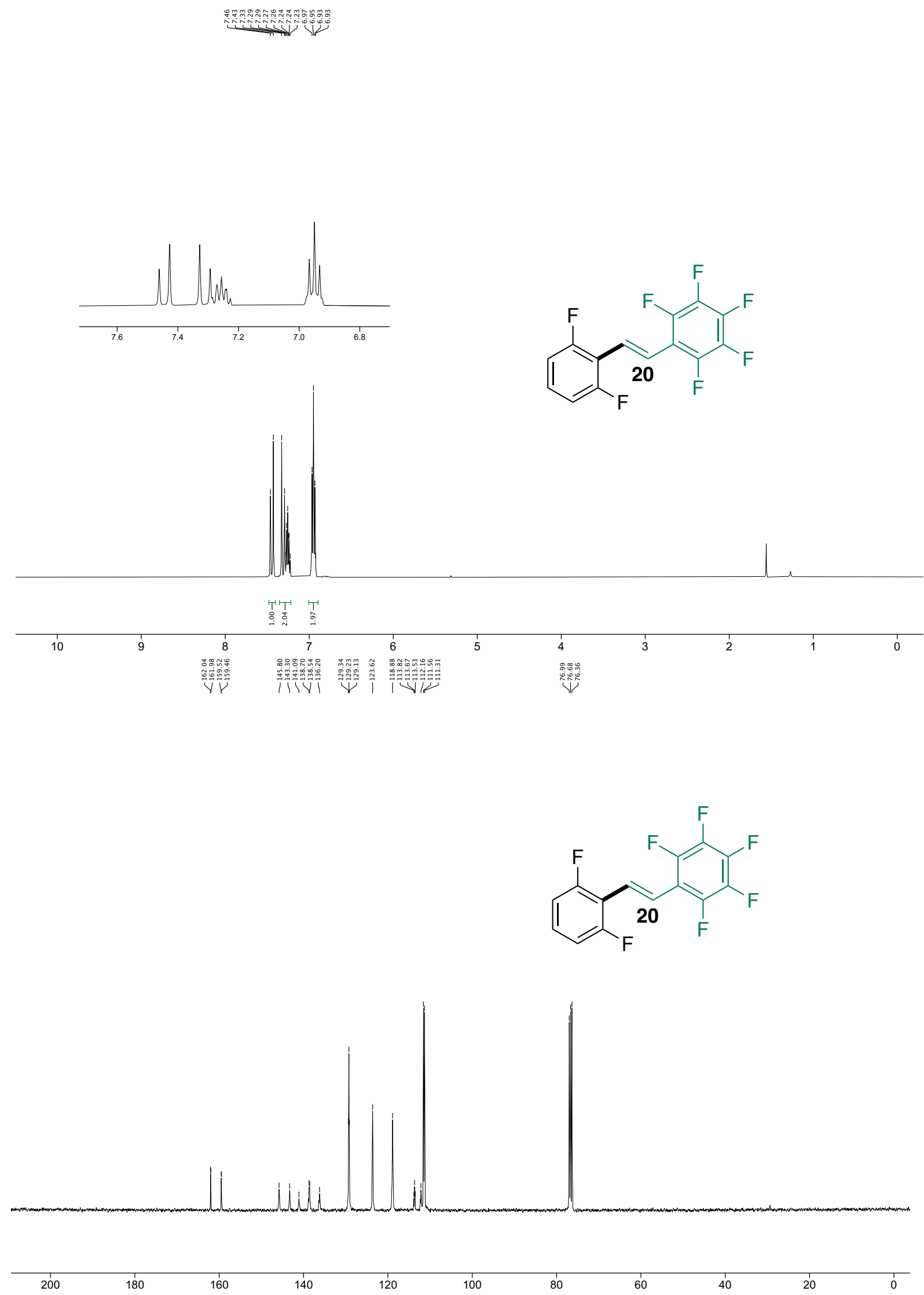

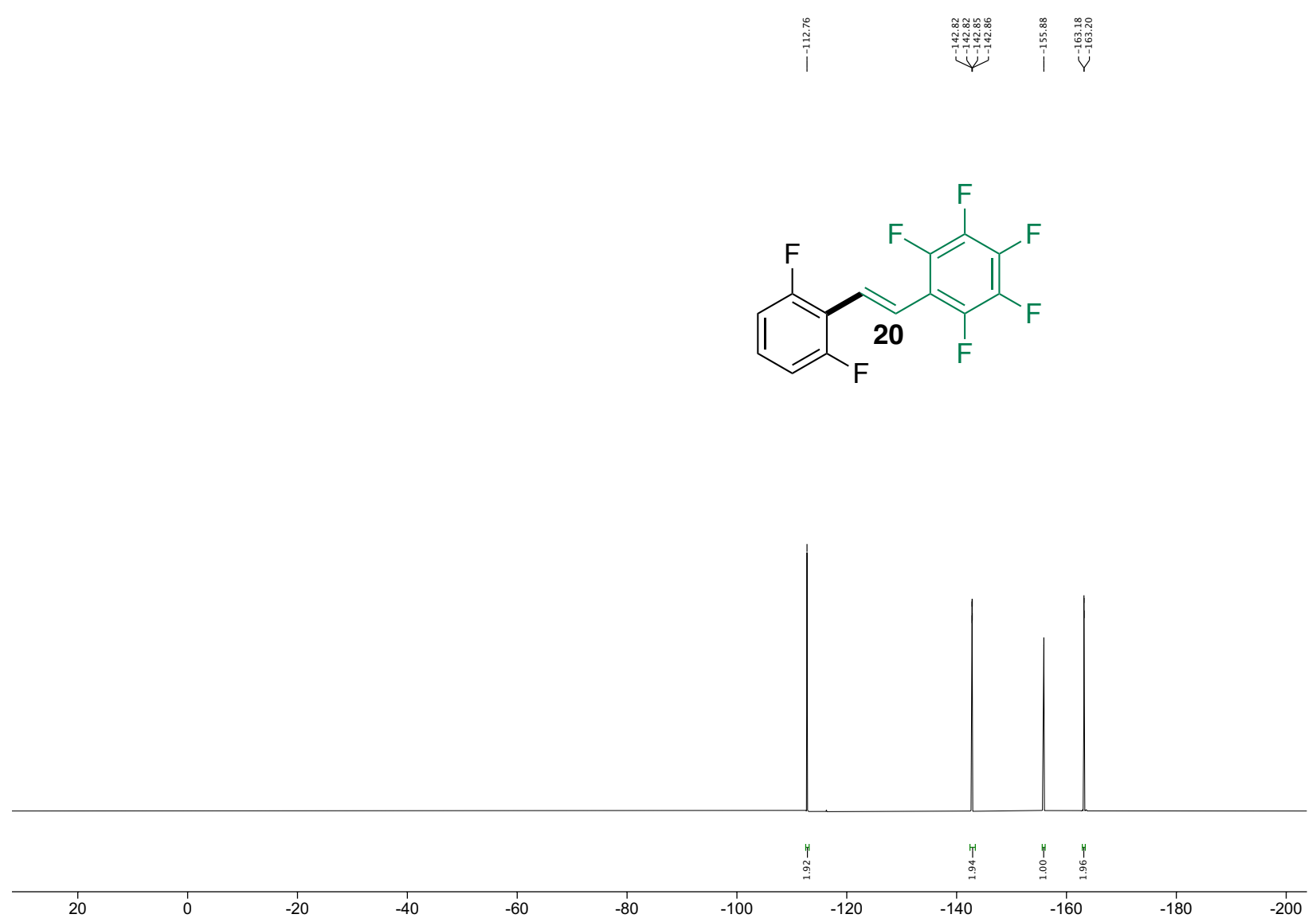


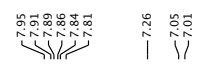<smiles>Fc1c(F)c(F)c(/C=C/c2ccc(C(F)(F)F)cc2C(F)(F)F)c(F)c1F</smiles>
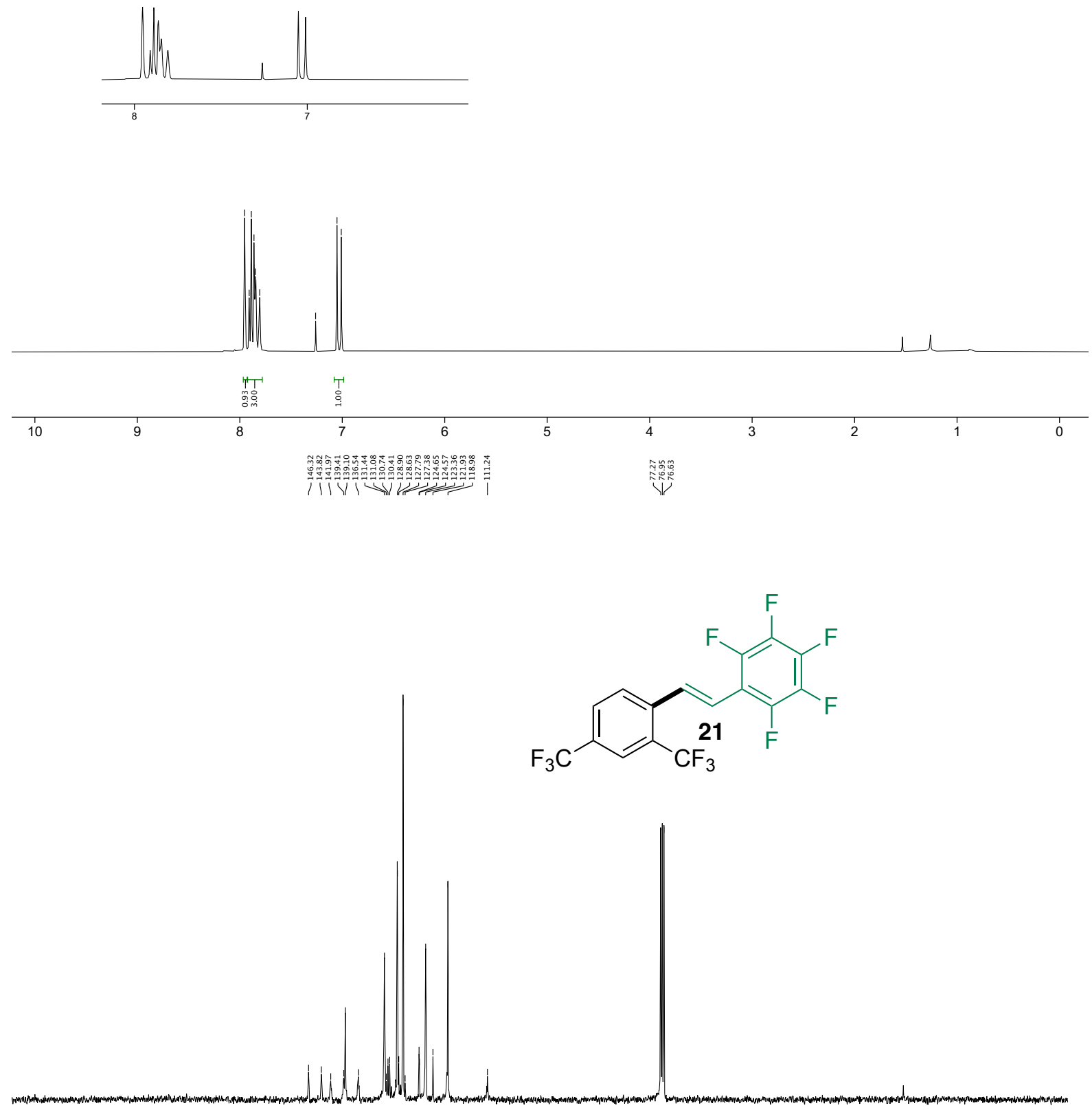

200

180

160

140

120

100

80

60

40

20 

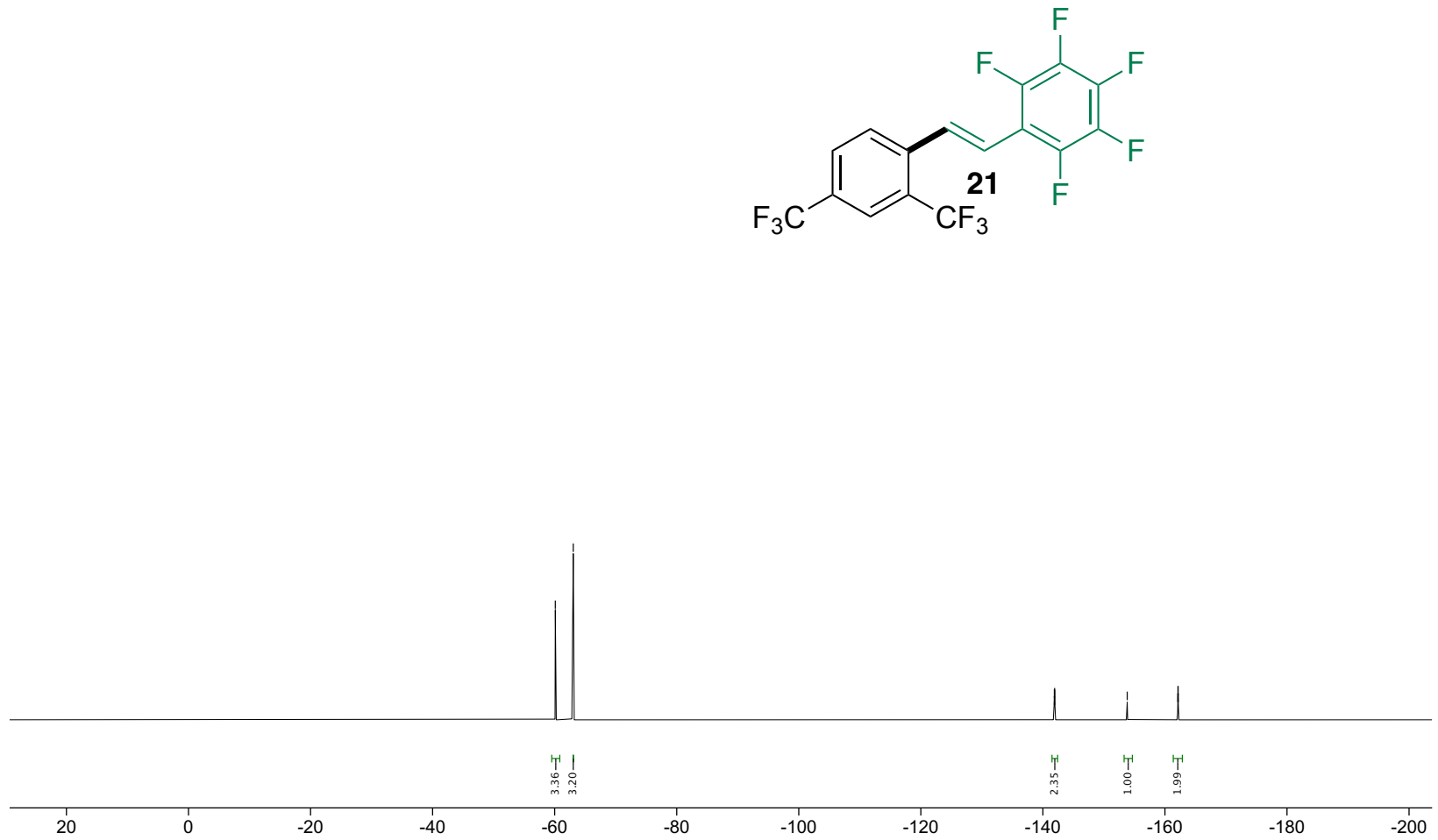

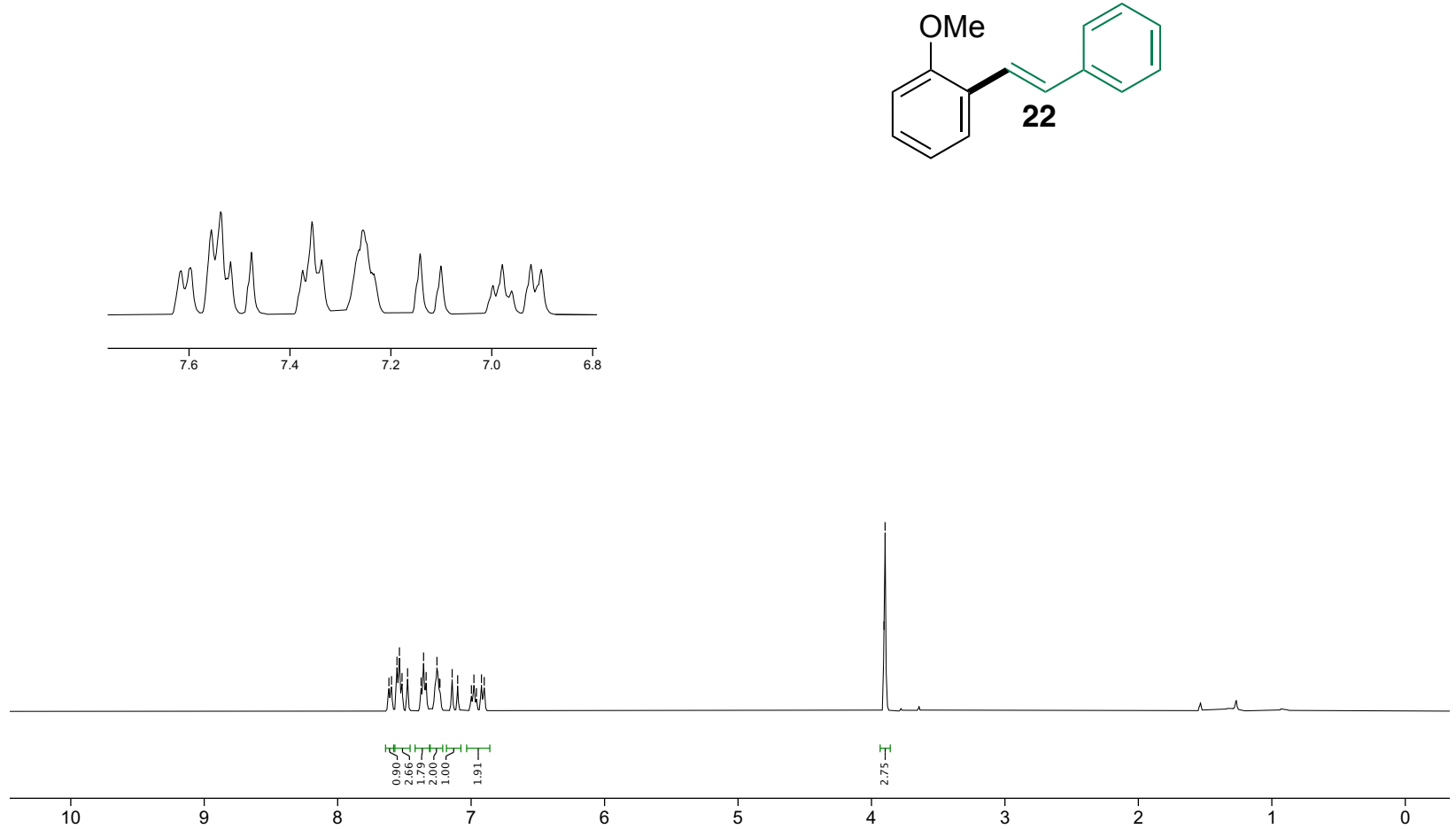


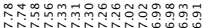

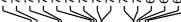

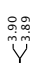

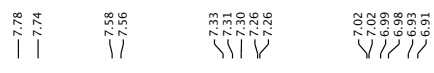
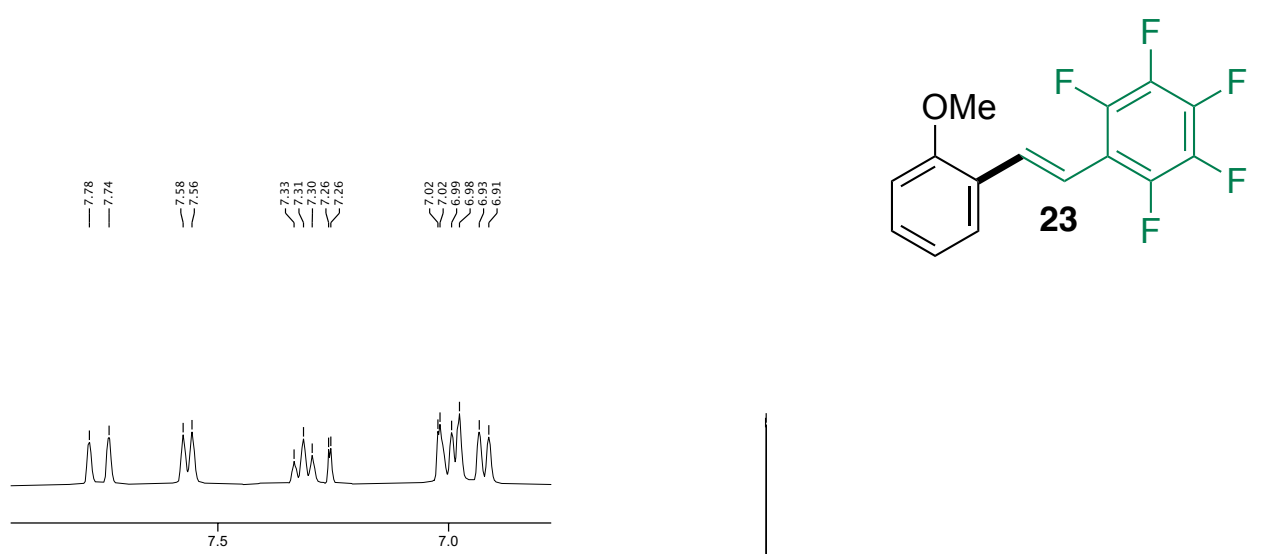

$\|$
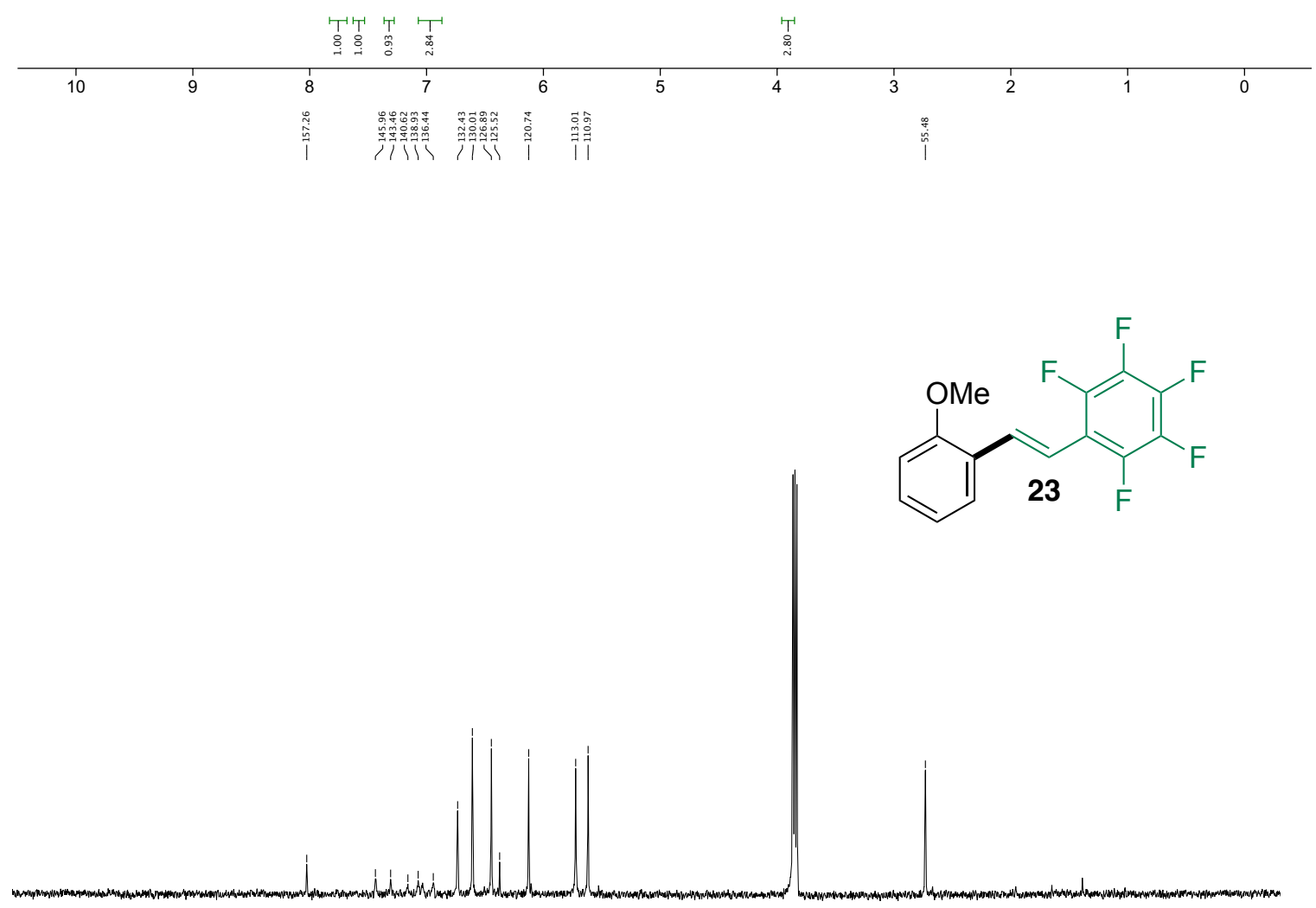

200

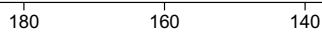

120

100

80

60

40

$20 \quad 0$ 

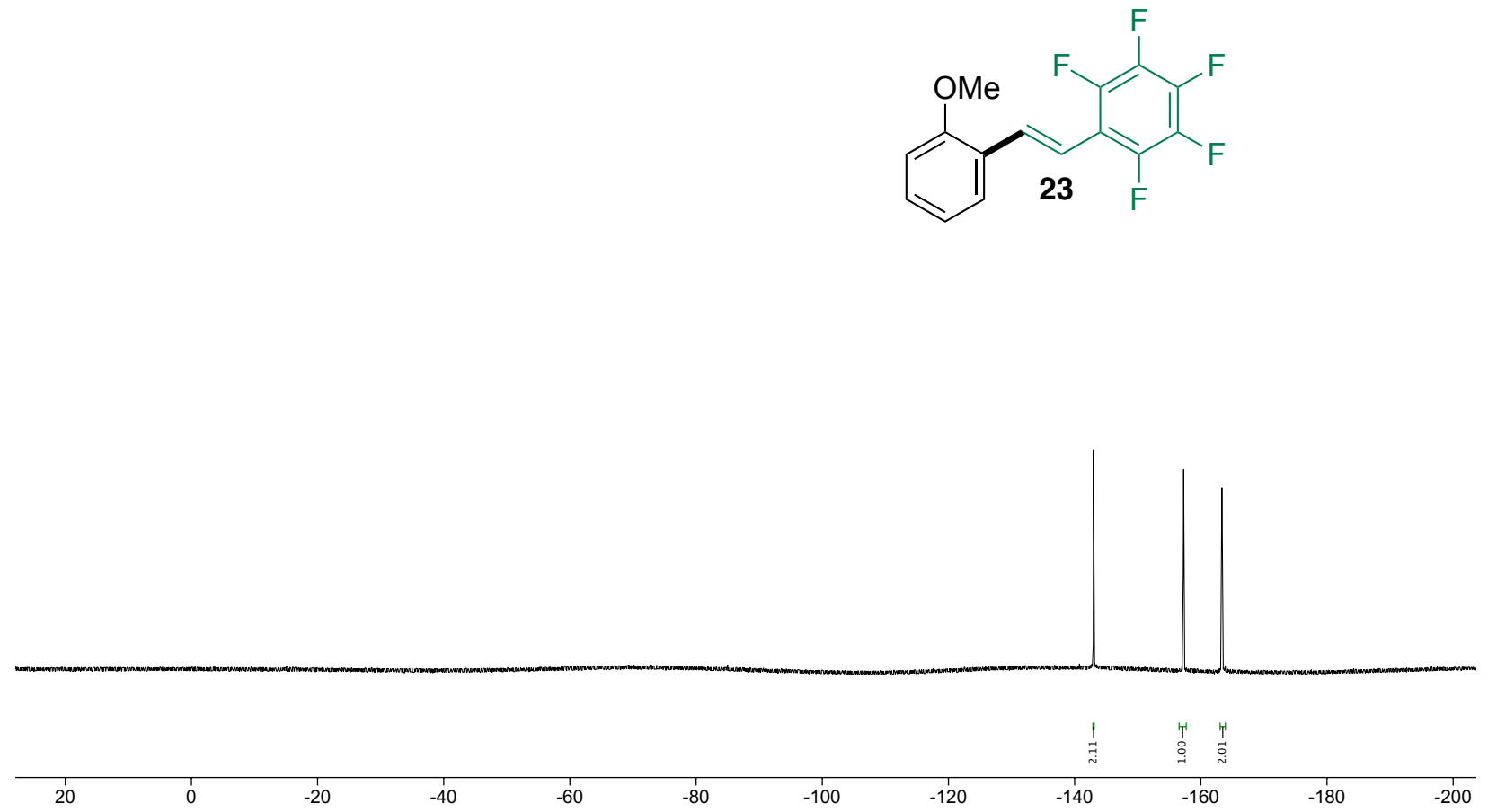


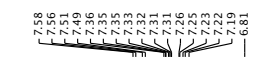
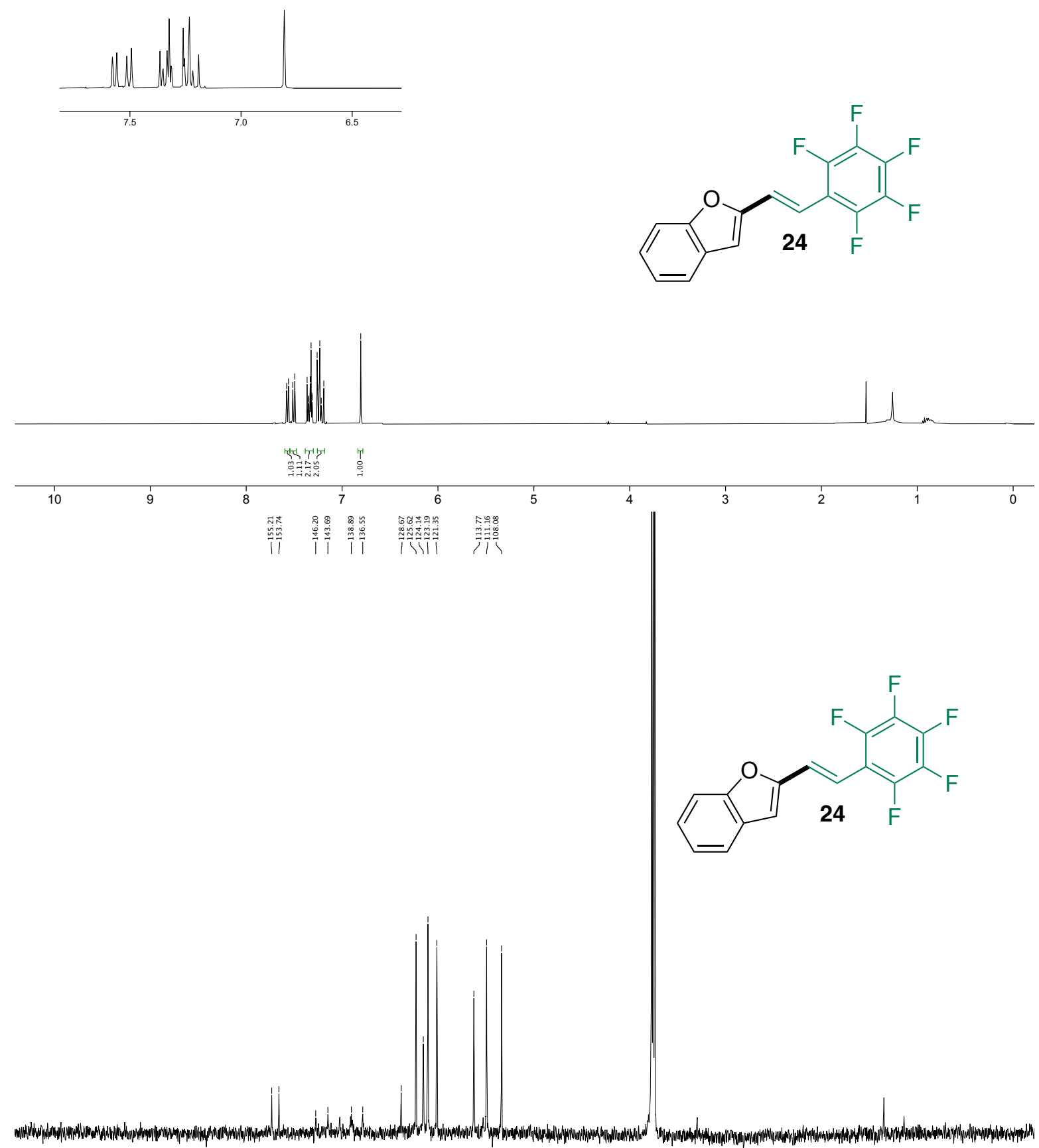

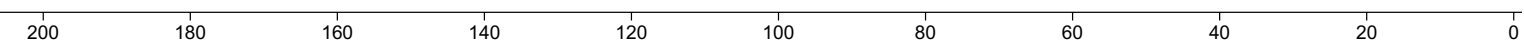



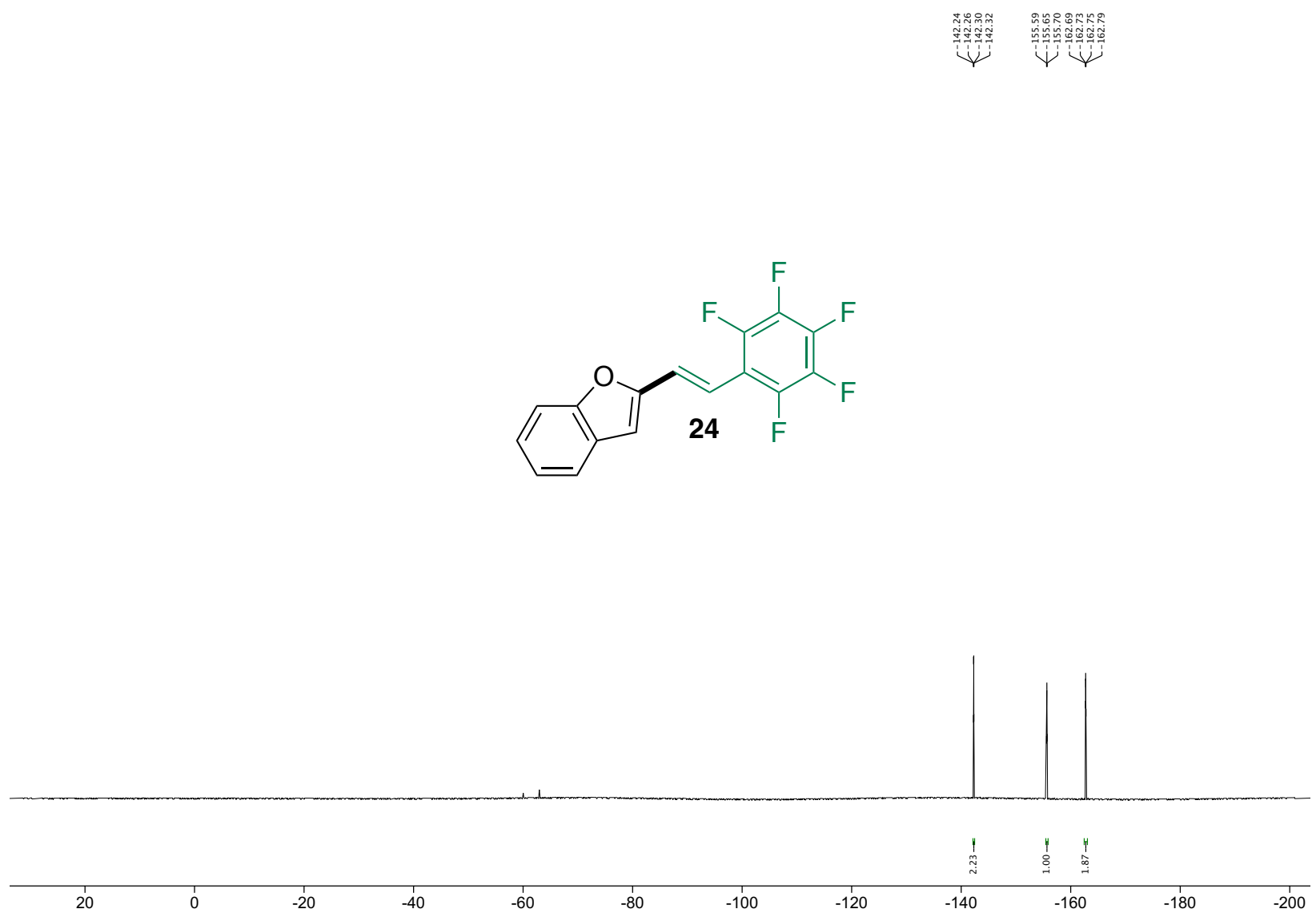

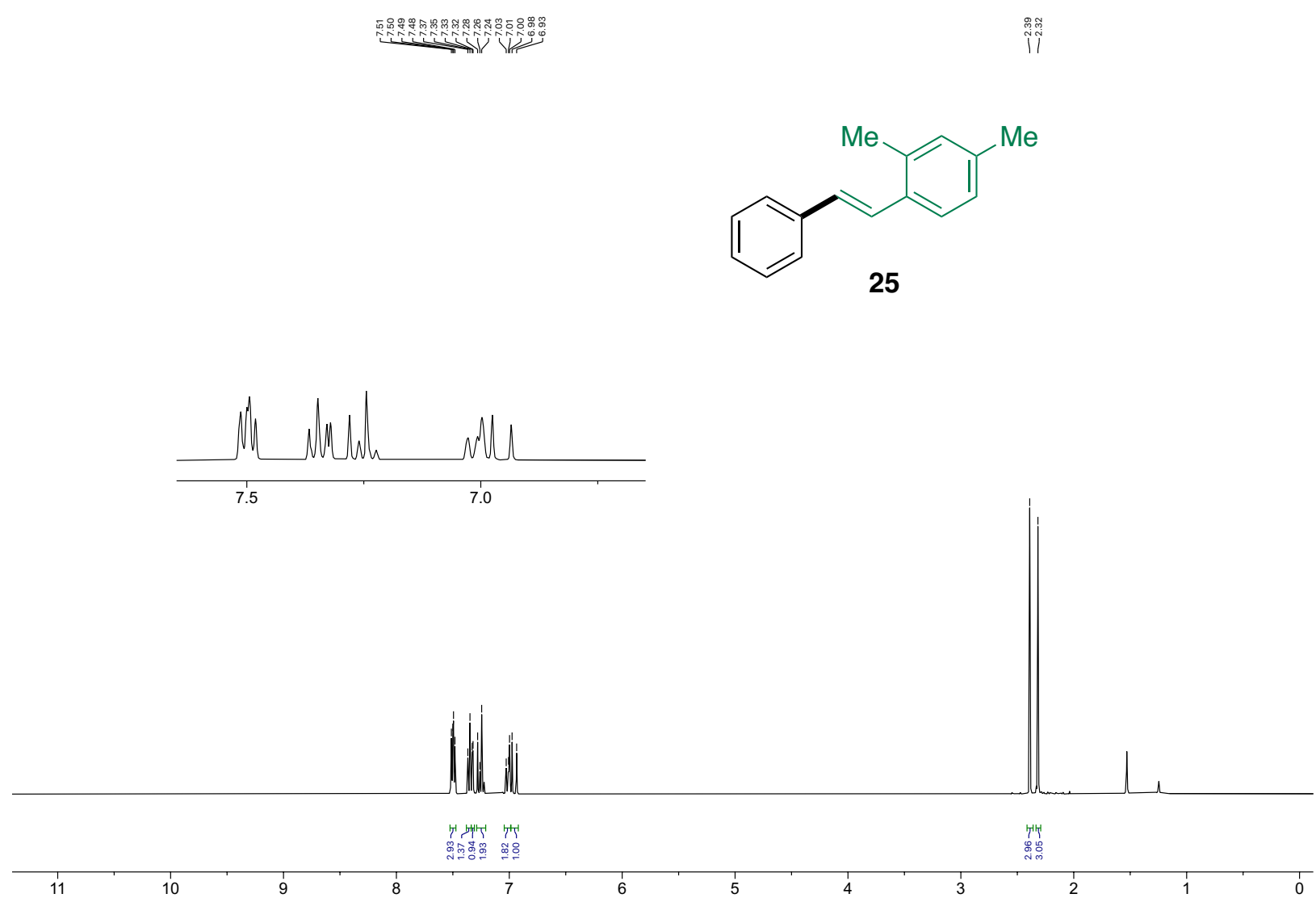


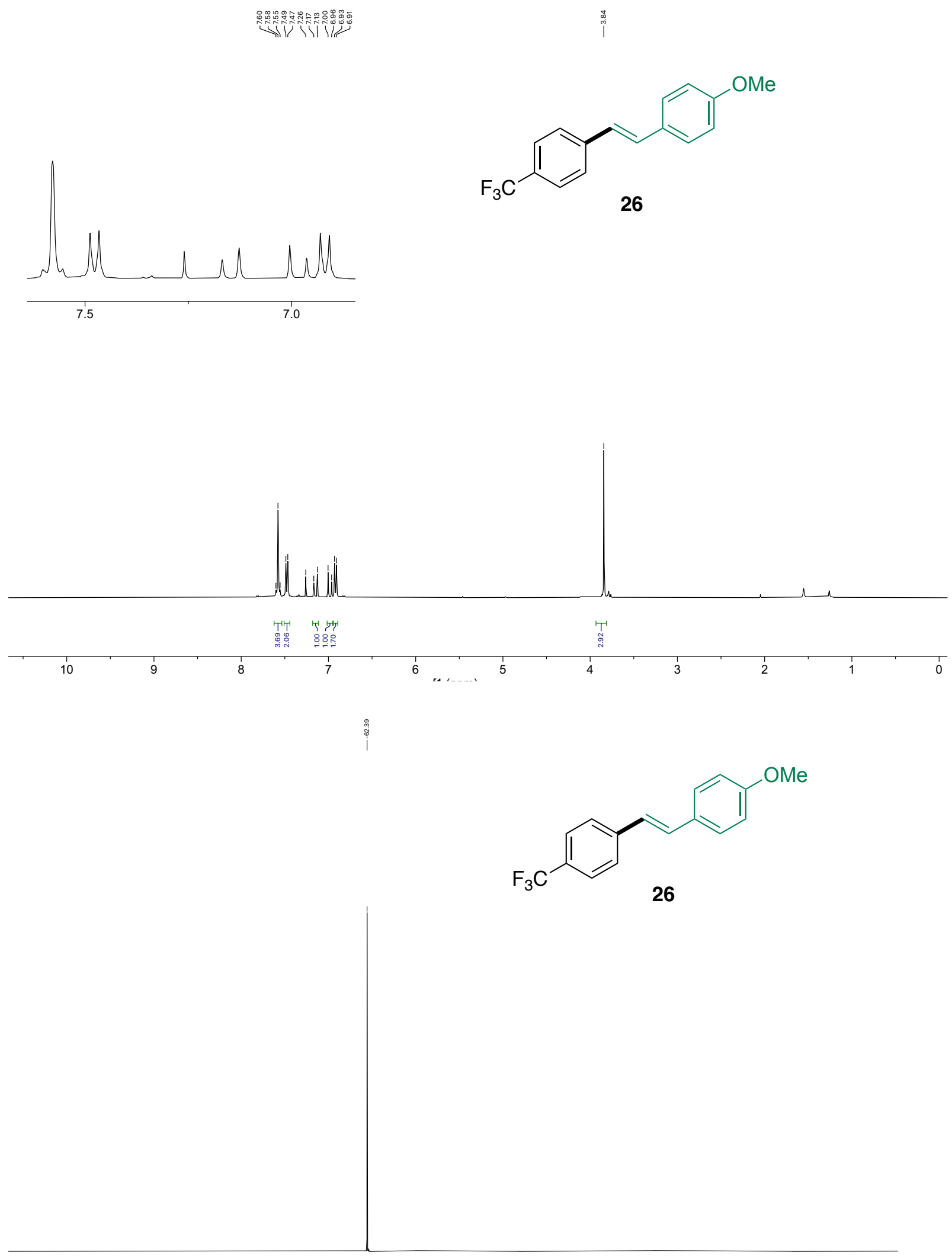

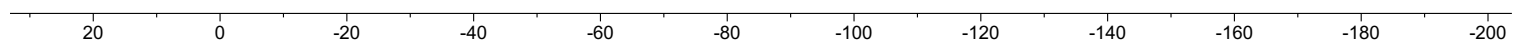




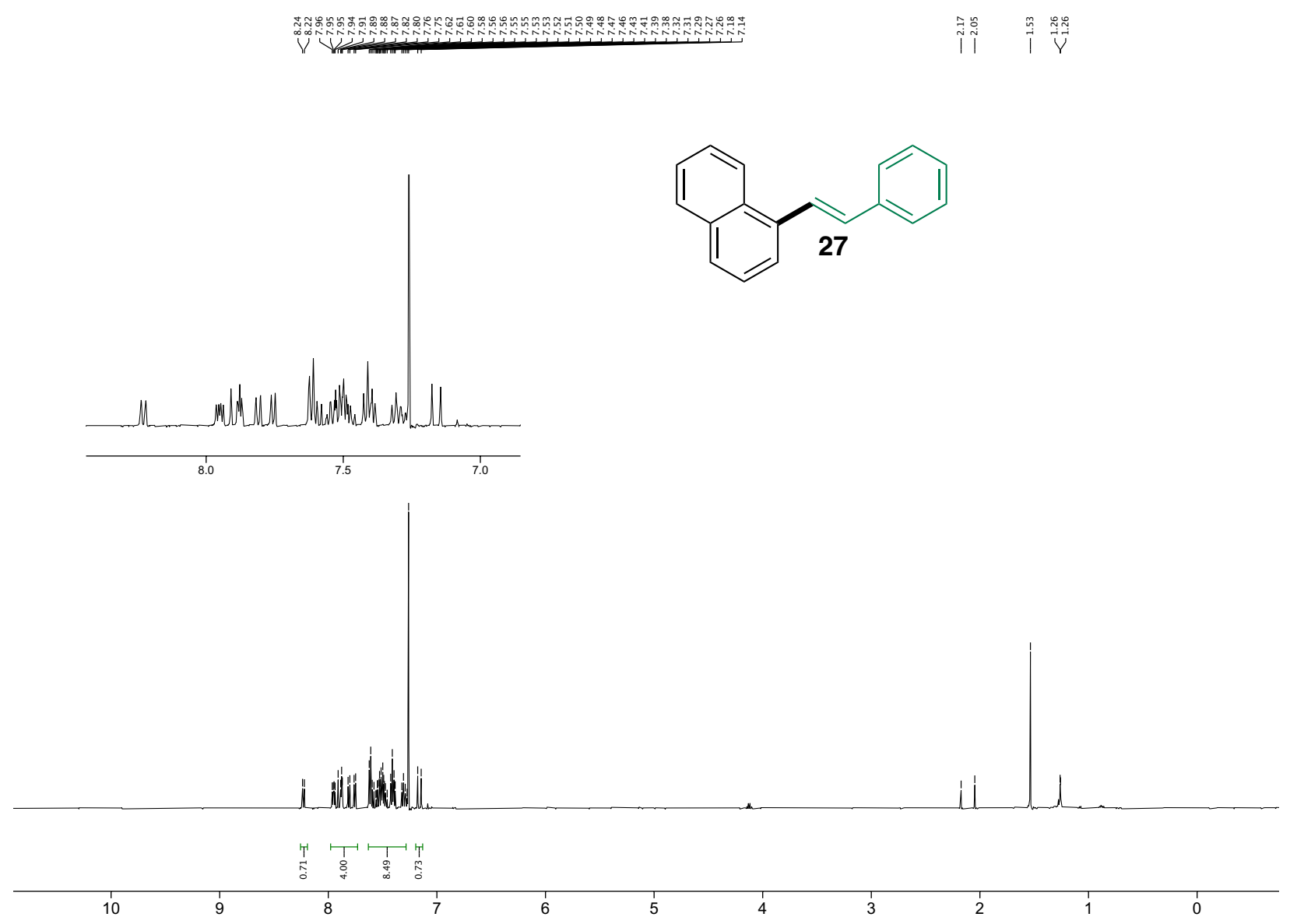




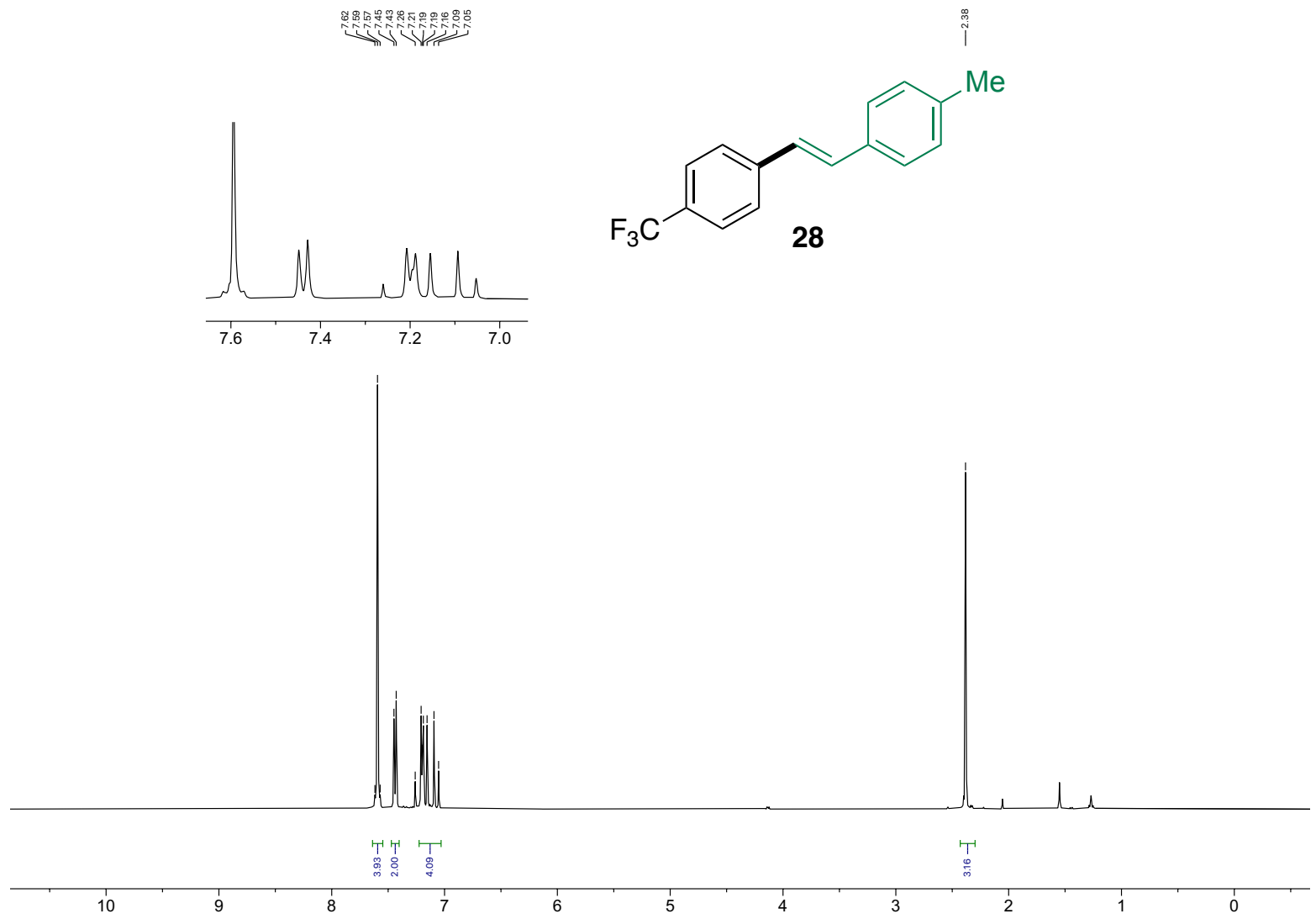



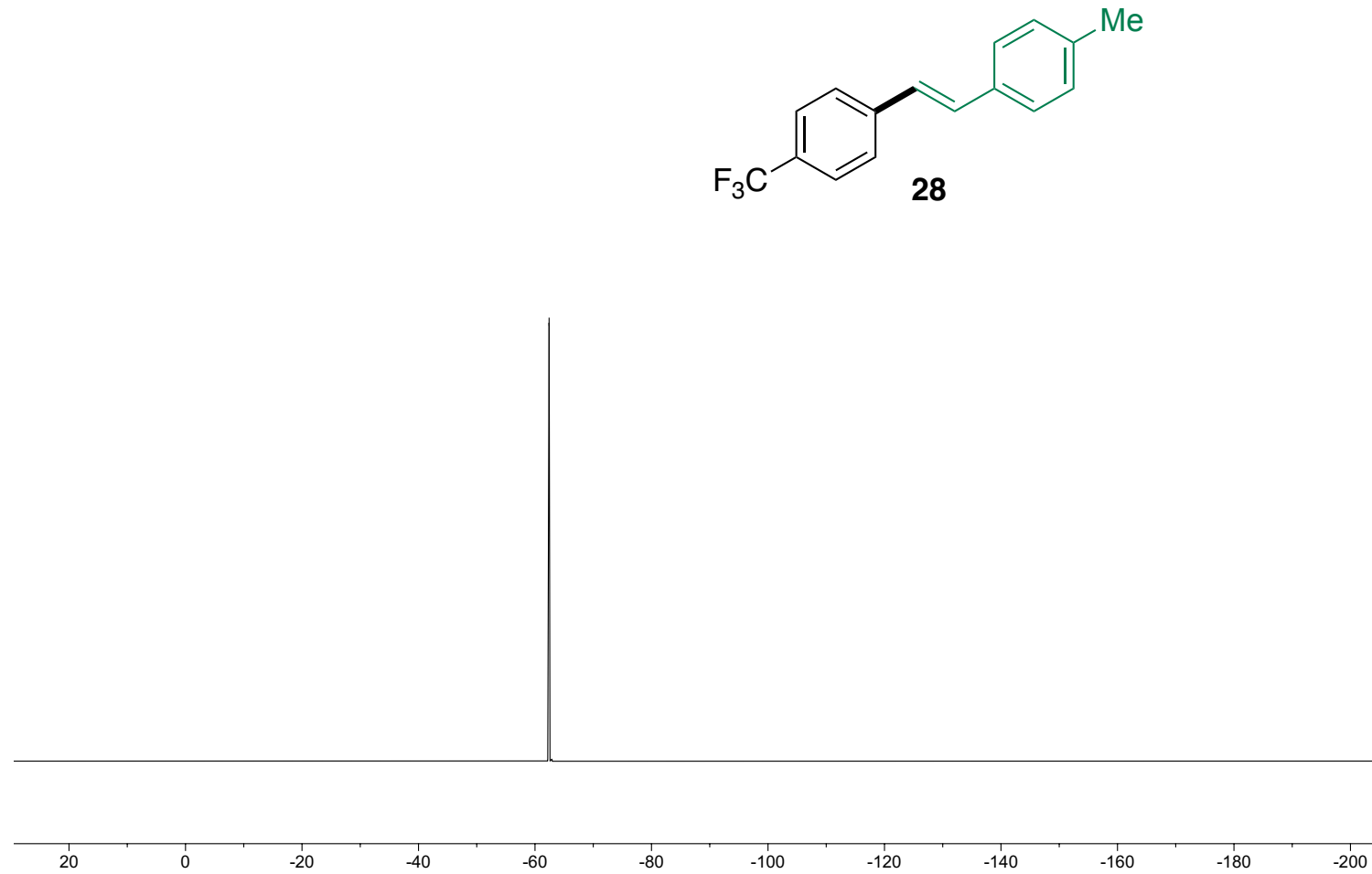

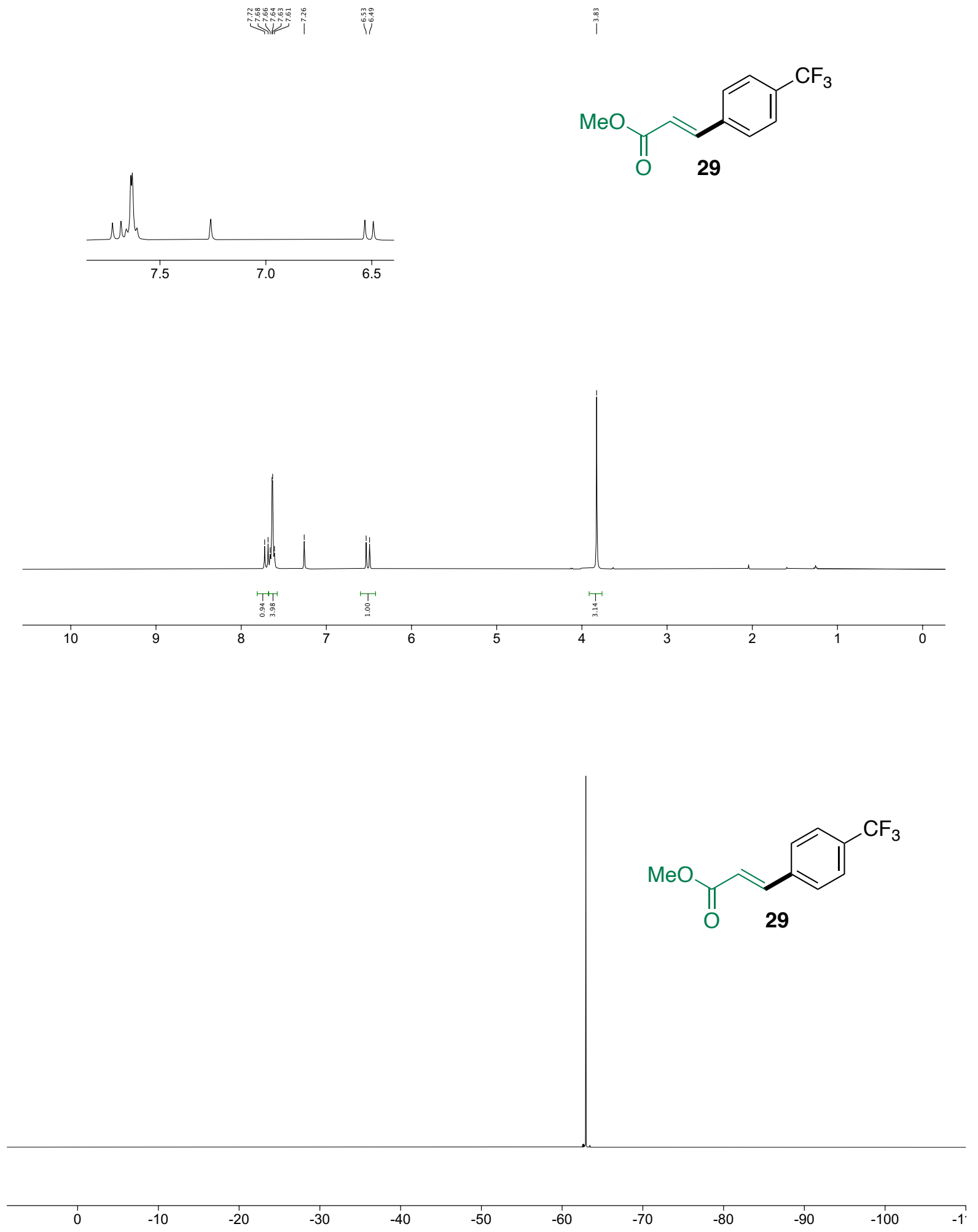


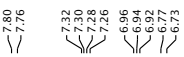
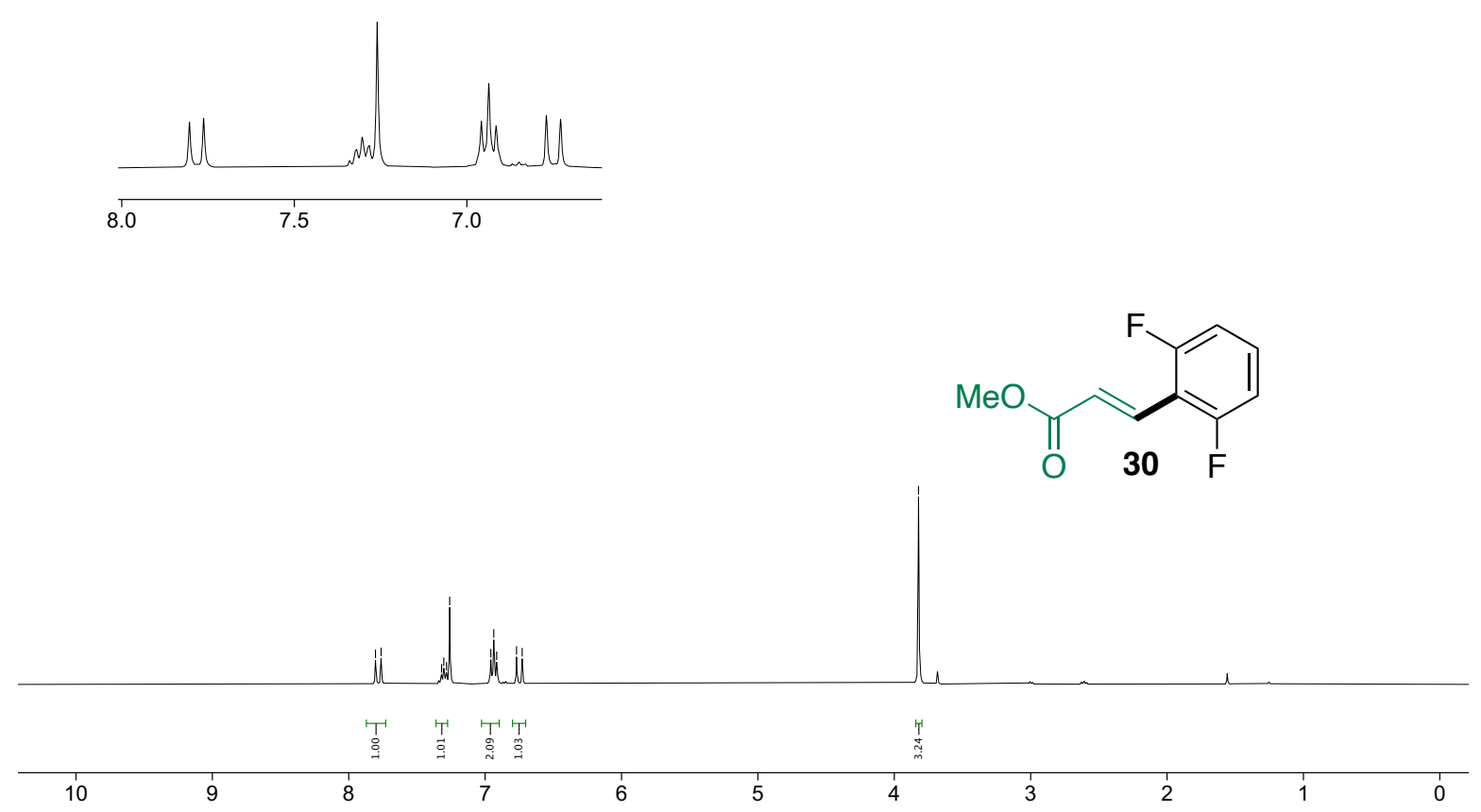

$\stackrel{\substack{0 \\ 0}}{7}$<smiles>COC(=O)/C=C/c1c(F)cccc1F</smiles>

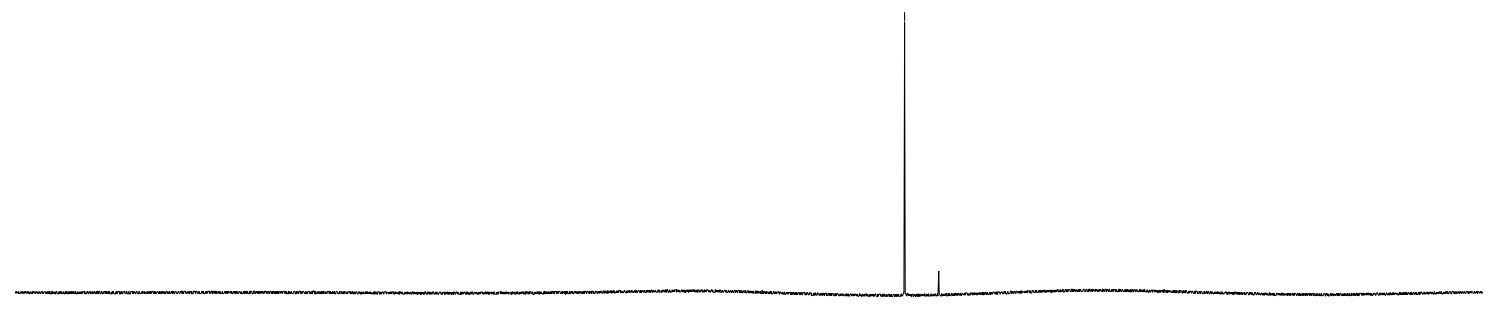




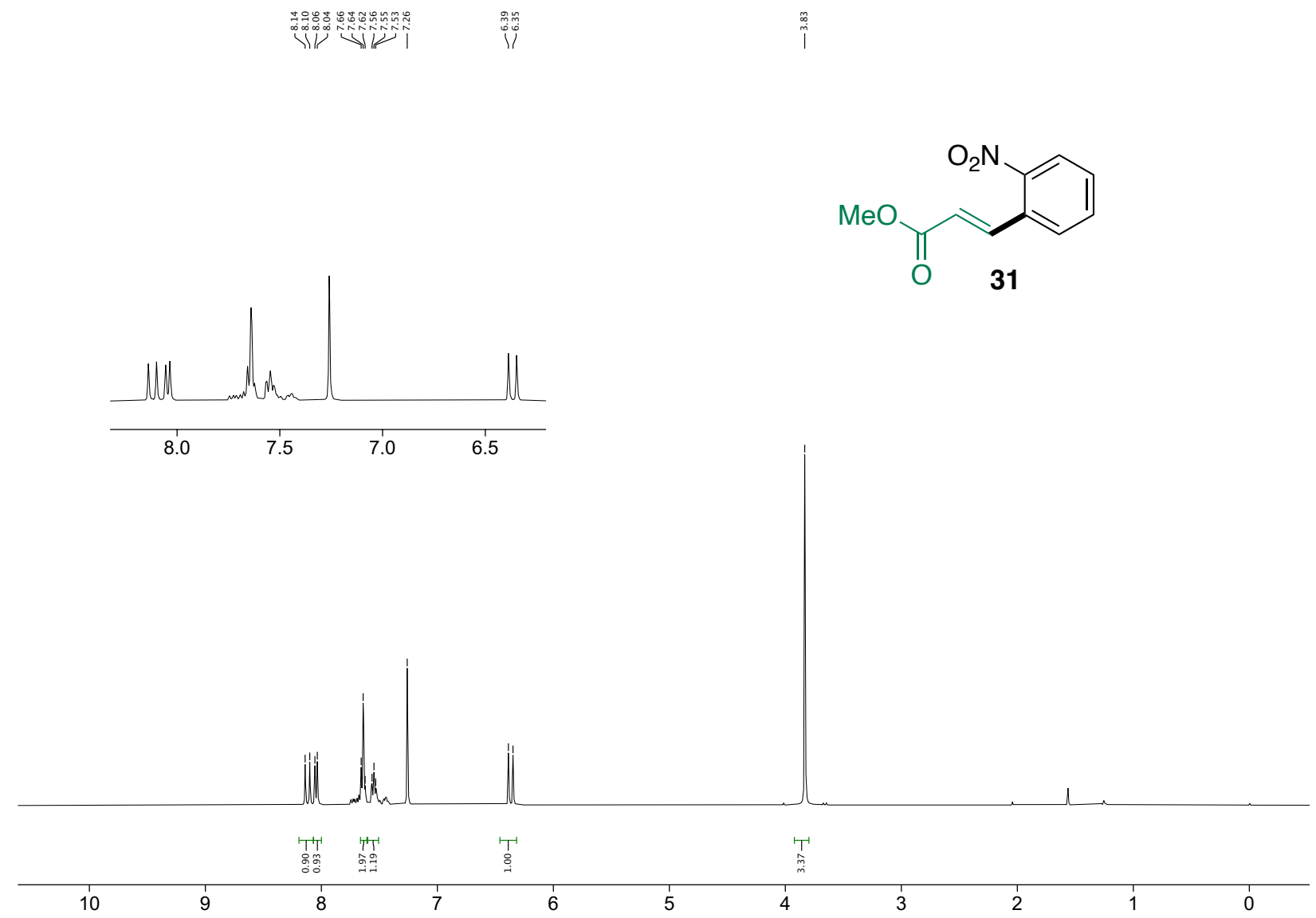




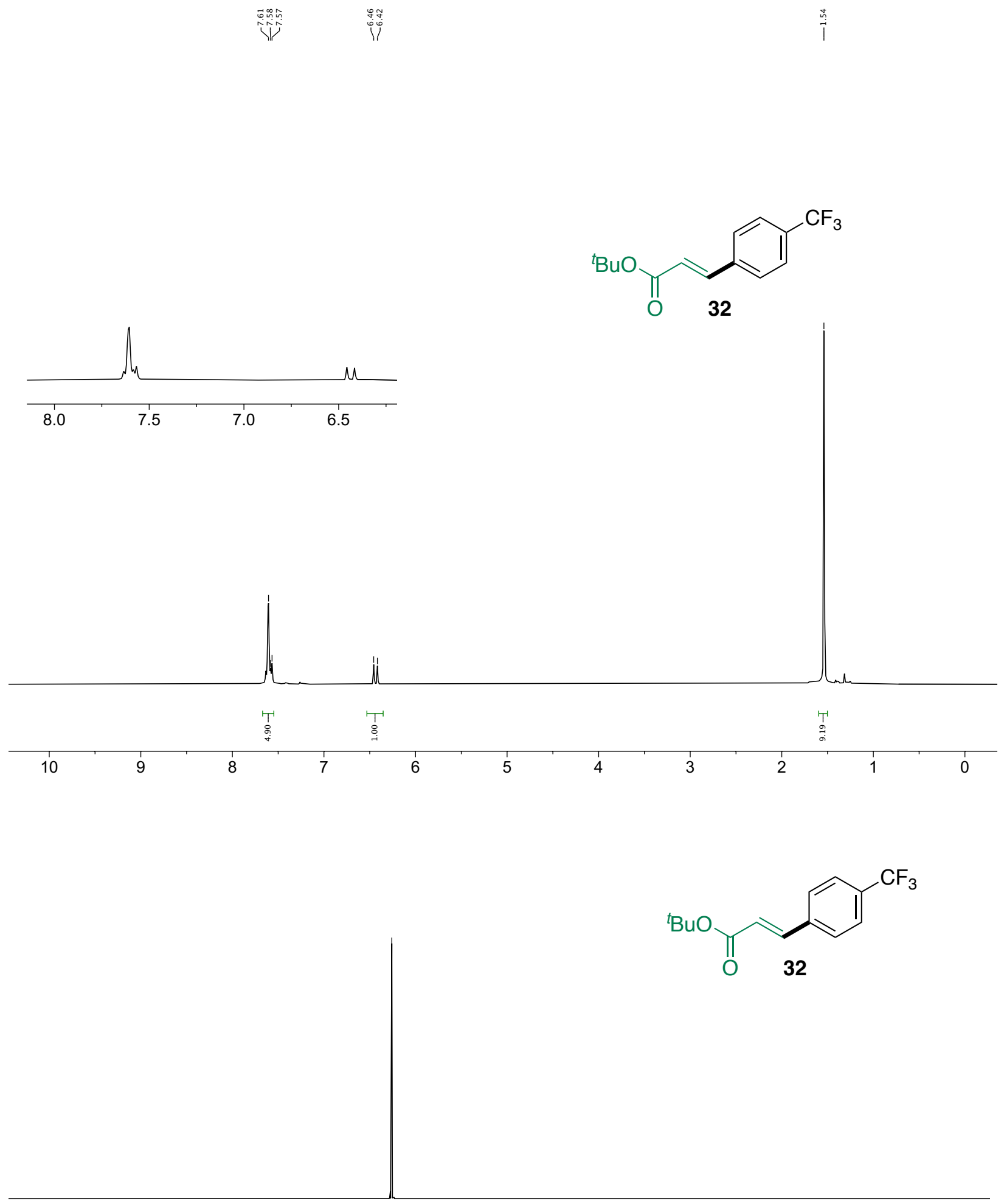

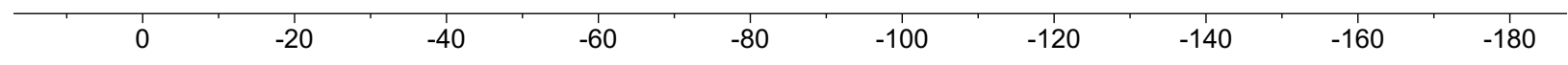

